\title{
2 Corinthians
}

Incipit

Present: $5^{1} 545^{86164757678778889}$

incipit ad eosdem secunda ] iterum ad corinthios 51, om. 54586164 , incipit ad corinthios secunda 75767778 , incipit textus epistulae secunda ad corinthios $88^{*}$, incipit epistula pauli ad corinthios secunda $88^{\mathrm{C}}$, incipit eiusdem secunda incipit epistula 89

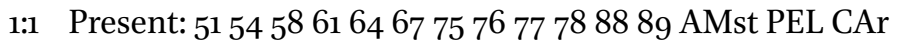

paulus apostolus $\langle 67\rangle$

iesu christi $\left.\mathrm{AMst} \mathrm{AD}^{\mathrm{AD}} \mathrm{PLL}^{\mathrm{var}}\right]$ christi iesu $5861 \mathrm{AMst}^{\mathrm{ed}} \mathrm{PEL}^{\mathrm{AB}}$, lac. 67, om. 78 per uoluntatem dei $54 * 64$ (75) ] lac. 67 et timotheus frater ecclesiae dei $5154586164\langle 67\rangle 7589]$ abs. CAr quae est $51545861\langle 67\rangle 88$ ] usitanti 77 , abs. CAr corinthi $(54) 58\langle 67\rangle]$ ] corinthis 61 , in corintho uel corinthi 77 , abs. CAr cum sanctis omnibus ] abs. CAr

qui sunt ] usitantibus 77, abs. CAr in uniuersa achaia ] in uniuersa ecclesia PEL ${ }^{\mathrm{var}}, a b s$. CAr

1:2 Present: $5^{1} 545^{8} 6164677576777888$ 89 AMst PEL SPM

gratia uobis ] + et misericordia SPM

et pax a deo ] + et $S \mathrm{M}^{\mathrm{var}}$

patre nostro ] patre $\mathrm{PEL}^{\mathrm{A}}$

et ] a PEL var

domino iesu christo ] domino nostro iesu christo $\mathrm{AMst}^{\mathrm{var}} \mathrm{SPM}^{\mathrm{ed}}$

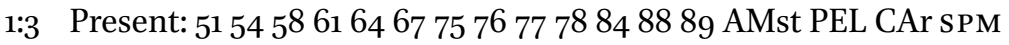

benedictus ] fratres benedictus $84 ;+$ est AMst ${ }^{\text {ed }}$ + es AMst ${ }^{\text {var }}$

deus ] dominus SPM ${ }^{\text {var }}$ + noster PEL ${ }^{\text {var }}$

et pater $\langle 67\rangle \mathrm{SPM}^{\mathrm{var}}$ ] pater 5458 , om. $89 \mathrm{PEL}^{\mathrm{var}} \mathrm{SPM}^{\mathrm{ed}}$

domini nostri ] $\mathrm{d}[\ldots] 67$, domini $\mathrm{SPM}^{\mathrm{var}}$

iesu christi ] lac. 67, abs. 84 ; + et 61

pater misericordiarum ] pater miserationum 64, abs. 84 CAr, pater gloriae SPM

et deus totius consolationis 5461 ] et deus omnis consolationis 647576 AMst, et deus totius uel omnis consolationis 77 , abs. 84 CAr SPM 
1:4 Present: 515458616467757677788889 AMst PEL

qui consolatur nos in 61

omni tribulatione $\langle 67\rangle]$ omni pressura $61 \mathrm{AMst}^{\mathrm{PEL}} \mathrm{B}^{\mathrm{B}}$

nostra ] lac. 67

ut possimus $\langle 67\rangle$ ] possimus $89^{*}$, ita ut possimus $89^{\mathrm{C}}$, ut possemus $\mathrm{PEL}^{\mathrm{var}}$

et ipsi consolari 61 ] nos consolari 6477 , et nos consolari 757689 AMst

eos qui ] lac. 67

in omni pressura sunt $\langle 67\rangle 7888$ ] sunt in omni angustia $61 \mathrm{PEL}^{\mathrm{B}}$, in omni tribulatione sunt 64757689 , in omni tribulatione uel pressura sunt 77 , sunt in omni pressura AMst

per exhortationem $54\langle 67\rangle 7888$ ] per consolationem 6164

qua ] quam $64 \mathrm{PEL}^{\text {var }}$, lac. 67 , quae 89 * PEL ${ }^{\text {var }}$

exhortamur 647888 ] consolamur $61,[\ldots]$ mur 67 , exhortabamur $89^{*}$, exhortatur PEL var

et ipsi a deo $\mathrm{AMst}^{\mathrm{var}}$ ] ipsi a deo $64 \mathrm{AMst}^{\mathrm{ed}}$, et ipsi a domino $\mathrm{PEL}^{\mathrm{B}}$

1:5 Present: 515458616467757677788889 AMst PEL

quoniam $\mathrm{AMst}^{\mathrm{AD}}$ ] quia $\mathrm{AMst} \mathrm{ed}^{\mathrm{ed}}$

sicut abundant $51545861\langle 67\rangle 88$

passiones christi ] lac. 67 , christi passiones AMst ${ }^{\mathrm{var}} \mathrm{PEL}^{\mathrm{var}}$

in nobis ] in uobis $7576 \mathrm{AMst}^{\mathrm{var}} \mathrm{PEL}^{\mathrm{var}}$

ita et per christum ] ita per christum (61), sic per christum 64 AMst

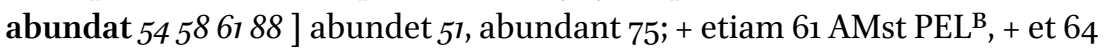

757677

consolatio nostra 61 ] lac. 67

1:6 Present: 5154586164677576777888 89 AMst PEL

siue autem (89) ] siue $61^{*},[\ldots]$ tem 67

tribulamur pro ] angustiam patimur pro $\mathrm{AMst}^{\mathrm{var}} \mathrm{PEL}^{\mathrm{B}}$, pressuram patimur pro AMst ${ }^{\text {ed }}$

uestra exhortatione 547888 ] uestra consolatione 6164 , lac. 67 , uestra aedificatione 89

et salute (89) ] om. $54^{*}$, lac. 67

siue exhortamur $\left.88 \mathrm{PEL}^{\text {var }}\right]$ siue consolamur $515888^{\text {alt }}$, om. (cf. 1:7) $54^{*} 61$

$7576777889 \mathrm{PEL}^{\mathrm{B}}$, siue obsecramus 64 , lac. 67, siue exhortationem consequimur AMst

pro uestra exhortatione $5^{c} 88$ ] pro uestra consolatione siue exhortamur pro uestra exhortatione 51, om. (cf. 1:7) $54^{*} 6175767778$ 89, pro uestra consolatione exhortatione 58 , pro uestram consolationem 64 , lac. 67, prọ ụẹ̦trạa consolatione $88^{\text {alt }}$; + et salute 515888 
quae operatur 51545861777888 ] lac. 67, om. $\mathrm{PEL}^{\mathrm{B}}$

in tolerantia ] tolerantia $5^{1} \mathrm{PEL}^{\text {var }}$, tolerantiam $5^{8} 61$, in sustentationem 64

757689 , lac. 67 , in sustentatione 77 , per patientiam AMst, in tolerantiam $P E L^{A}$, sufferentiam PEL ${ }^{B}$

earundem passionum (54) (89) ] earum passionum 61, eorumdem passionum 64, lac. 67

quas ] lac. 67; + uel sicut 77

et nos patimur ] lac. 67, nos patimur 88*, et nos ipsi patimur ipsi AMsted

1:7 Present: 51545861647576777888 89 AMst PEL

et spes nostra ] ut spes nostra $61 \mathrm{AMst}^{\mathrm{R}}$

firma ] certa AMst; + est $5^{1}\left\langle 54^{*}\right\rangle 5^{86188^{*}} \mathrm{AMst}^{\mathrm{ed}} \mathrm{PEL}^{\mathrm{B}},+$ sit $\mathrm{AMst}^{\mathrm{R}} \mathrm{PEL}^{\text {var }}$ pro uobis ] pro nobis AMst ${ }^{\text {var }} \mathrm{PEL}^{\mathrm{B}}$; + est 64

\pm siue exhortamur pro uestra exhortatione et salute 6175767778 89, siue consolationem consequimur pro uestri consolatione et salute $\mathrm{AMst}^{\mathrm{R}}$

scientes ] quia scimus AMst

quoniam ] quia $616475767789 \mathrm{PEL}^{\mathrm{B}}$, quod AMst, om. PEL ${ }^{\text {var }}$

sicut $\mathrm{AMst}^{\mathrm{R}}$ ] om. 6477 , si $757689 \mathrm{AMst}^{\mathrm{ed}}$, et si PEL ${ }^{\mathrm{B}}$

socii passionum estis ] passionum estis socii 61, socii passionum 757677

89 *, socii estis passionum AMst

sic ] om. 75767789 , simul PEL ${ }^{B}$

eritis et $\left(89^{C}\right)$ ] etiam 64, estis et $75767789^{*}$, et AMst PEL ${ }^{B}$

consolationis 61 ] consolationum $757689^{\mathrm{C}}$, resurrectionis $\mathrm{PEL}^{\mathrm{B}}$; + eritis 75 76 AMst $^{\text {ed }}$ PEL $^{\mathrm{B}}$

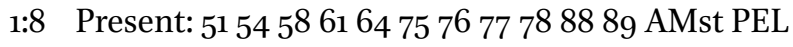

non enim uolumus

ignorare uos ] uos ignorare $5164757677 \mathrm{AMst}^{\mathrm{var}} \mathrm{PEL}$ var, ignorare 78 PEL $^{\mathrm{B}}$

fratres de tribulatione ] fratres de passione 51, fratres de pressura 61 AMst $P E L^{B}$

nostra quae facta est 5154586188 ] nostra facta 77 , quae nobis facta est $\mathrm{AMst}^{\mathrm{R}}$, uestra quae facta est $\mathrm{PEL}^{\mathrm{B}}$

in asia 5161 ] in domino 78

quoniam ] quia 64757689 AMst PEL var

supra modum ] ultra modum 64

grauati sumus ] om. 64, onerati sumus $89 \mathrm{PEL}^{\mathrm{B}}$, grauati fuimus $\mathrm{AMst}^{\mathrm{R}}$; + uel onerati 77

supra ] super 757689

uirtutem ] uires $6164 \mathrm{AMst}^{\mathrm{PEL}}{ }^{\mathrm{B}}$; + grauati sumus 64, + nostras $\mathrm{PEL}^{\mathrm{B}}$ 


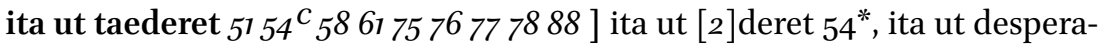
remus AMst, ita ut cedet $P E L^{B *}$, ita ut taedent $P E L^{B C}$

nos etiam uiuere ] et nos uiuere 58 , nos uiuere $61 \mathrm{PEL}^{\text {var }}$

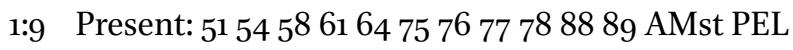

sed ipsi in ] sed si ipsi in 51, quin et ipsi in $\mathrm{AMst}^{\mathrm{R}}$, sed ipsi $\mathrm{PEL}^{\mathrm{B}}$

nobis ipsis ] nobis $5^{1} 6177$ AMst PEL $^{\mathrm{B}}$, nobismet ipsis $5^{864}$

responsum mortis habuimus ] responsum mortis accepimus $\mathrm{AMst}^{\mathrm{R}}$

ut non $\langle 64\rangle$

simus fidentes in nobis ] simus confidentes in uobis 58 , fidentes in nobis simus (64), fidentes simus in nobis 77, essemus fidentes in nobis $\mathrm{AMst}^{\mathrm{R}}$

sed in deo ] sed in domino $5461757688^{*}$ AMst $^{\text {var }}$ PEL $^{\text {var }}$, sed in deum 7789 qui suscitat ] suscitantem 77 , qui suscitabit $A M s^{\text {var }}$, qui suscitauit AMst ${ }^{\text {var }}$ PELvar

mortuos ] a mortuis AMst ${ }^{\text {var }}$

1:10 Present: $51545^{8} 61647576777888$ 89 AMst PEL

qui de tantis periculis 78 ] qui de tantis [...] 64, qui de tantis moribus 75 76 , qui de tanta uel periculo mortis 77 , qui de periculo $7^{\mathrm{gl}}$, qui de tantis mortibus AMst

eripuit nos ] lac. 64, liberauit nos AMst

et eruet ] et eruit $545^{8} 78 \mathrm{PEL}^{\mathrm{var}}$, om. $61757689 \mathrm{AMst}^{\mathrm{ed}} \mathrm{PEL}^{\mathrm{A}}$, lac. 64, et eripiet 77 , et liberat $\mathrm{AMst}^{\mathrm{R}}$

in quem $\mathrm{AMst}^{\mathrm{R}}$ ] lac. 64, in quo $\mathrm{AMst}^{\mathrm{ed}}$; + et $\mathrm{PEL}^{\mathrm{B}}$

speramus ] sperauimus 58 , lac. 64

quoniam et ] lac. 64, et quoniam 77, quia et AMst ${ }^{\text {ed }}$, quia AMst ${ }^{\text {var }}$, quoniam PEL $^{\mathrm{B}}$

adhuc (88*) PEL var ] om. 6175767789 AMst PEL $^{\mathrm{A}}$, lac. 64; + et PEL ${ }^{\mathrm{B}}$

eripiet ] liberabit $61 \mathrm{AMst}^{\mathrm{ed}} \mathrm{PEL}^{\mathrm{B}}$, lac. 64, liberauit AMst ${ }^{\mathrm{var}} \mathrm{PEL}^{\mathrm{var}}$; + nos 61 PELvar

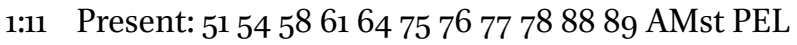

adiuuantibus ] cooperantibus 64, accedente $\mathrm{AMst}^{\text {ed }}$, accendentes AMst ${ }^{\mathrm{var}}$;

+ pro nobis AMst

et ] om. $58^{*} \mathrm{PEL}^{\text {var }}$, etiam $6164 \mathrm{AMst}^{\mathrm{PEL}}{ }^{\mathrm{B}}$

uobis ] uestra AMst

in oratione pro nobis ] in oratione pro uobis 58 , per orationem pro nobis 61 $\mathrm{PEL}^{\mathrm{var}}$, in orationem pro nobis 64 , pro nobis in oratione 7576 , de nobis oratione 77 , pro nobis in orationem 89 , oratione AMst, in oratione PEL ${ }^{\text {var, }}$ orationibus uestris PEL var 
ut ] om. 89

ex ] in 616475767789 AMst, om. PEL var

multis personis ] multarum personis facierum $5^{1} 88$, multorum personis $54^{*}$, multarum facierum $54^{\mathrm{C}} \mathrm{PEL}^{\mathrm{A}}$, multorum personis facierum 58 , multa facie 617789 , multorum faciem 64 , multifacie 7576 , multarum facierum personis 78 , multorum facie donum AMst, multorum facie $\mathrm{PEL}^{\mathrm{B}}$, multarum ex personis PEL var

eius ] om. 616475767789 AMst PEL $^{\mathrm{B}}$

quae 51545861767788 ] quod AMst; + est 61

in nobis est $61 \mathrm{AMst}^{\mathrm{A}}$ ] in nos $\mathrm{AMst} \mathrm{t}^{\mathrm{ed}}$, in uobis est $61^{\text {alt }} \mathrm{PEL} \mathrm{L}^{\mathrm{var}}$

\pm conlatum est AMst ${ }^{\text {ed }}$, latum est AMst ${ }^{\text {var }}$, consolatum est AMst ${ }^{\text {var }}$

donationis ] gratia 64757677 89, om. AMst, donatio PEL ${ }^{\text {var }}$, donationes PELvar

per multos

gratiae agantur $5^{1} 545^{8} 76$ ] gratiae referantur $61 \mathrm{PEL}^{\mathrm{B}}$, gratiae aguntur $6478 \mathrm{PEL}^{\text {var }}$, gratificetur 77 , in gratiarum actione celebretur $\mathrm{AMst}^{\mathrm{ed}}$, in gratiarum actiones celebretur $\mathrm{AMst}^{\mathrm{A}}$, in gratiarum actionem celebretur $\mathrm{AMst}^{\mathrm{var}} ;+$ deo PEL var

pro nobis ] pro uobis $54 \mathrm{PEL}^{\mathrm{B}}$

1:12 Present: 5154586164757677788889 AMst PEL CAr SPE

nam gloria nostra $\left.\left(64^{*}\right)\left(88^{*}\right)\right]$ gloria enim nostra 6177 AMst, nam exultatio nostra $\mathrm{PEL}^{\mathrm{B}}$, gloria nostra CAr

haec est $\left.51545^{8}\right]+$ et $5^{8}$

testimonium conscientiae nostrae 5154587888

quod ] quoniam $61 \mathrm{PEL}^{\mathrm{B}}$, quia $64 \mathrm{AMst}^{\mathrm{ed}}$, quanto AMst ${ }^{\mathrm{var}}$, abs. CAr in simplicitate 61 ] in sanctitatem 64 , in simplicitatem $89, a b s$. CAr et sinceritate ] et in sinceritate 61 , et sinceritatem 64, abs. CAr

dei ] om. AMst, abs. CAr et ] om. 6475767789 AMst PEL $^{\mathrm{A}}$, abs. CAr

non in sapientia carnali sed in gratia dei 5488 ] abs. CAr

conuersati sumus ] conseruati sumus 61, abs. CAr

in mundo ] in hoc mundo $51586175767888^{*} 89$ AMst PEL $^{\mathrm{B}} \mathrm{SPE}^{\mathrm{var}}$, abs. CAr abundantius autem 5154586188 ] abundantius PEL ${ }^{\text {var }}$, abs. CAr SPE ad uos ] apud uos AMst, abs. CAr SPE

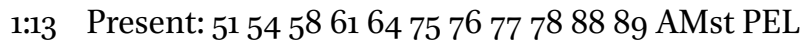
non enim alia ] non enim alias $75 \mathrm{AMst}^{\text {var }}$

scribimus uobis AMst ${ }^{\mathrm{ADR}}$ ] scripsimus uobis 6164 AMst $^{\mathrm{ed}}$, scribimus uel scripsimus uobis 77 , uobis scribimus PEL var 
quam quae 5154586177 ] namque $\mathrm{PEL}^{\mathrm{B}}$

legistis ] legitis $757689 \mathrm{AMst}^{\mathrm{R}}$

et $\left.\mathrm{AMst}^{\mathrm{var}}\right]$ uel AMst ${ }^{\text {ed }}$

cognoscitis $\langle 64\rangle$ ] cognouistis $5^{1} 6178$

spero autem ] spero enim PEL var

quod ] quo $54 \mathrm{PEL}^{\text {var, }}$, quoniam $6164757689^{\mathrm{C}}$, quoniam uel quod 77

usque in finem ] in finem usque $61\langle 64\rangle 89$, usque ad finem AMst ${ }^{\text {var }}$

cognoscetis AMst $\left.{ }^{\mathrm{var}}\right]$ cognoscitis $54^{*} 89 \mathrm{AMst}^{\mathrm{ed}} \mathrm{PEL}^{\mathrm{var}},[c]$ ogno[...] tis 64

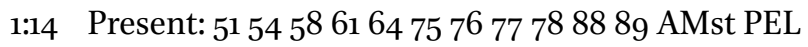

sicut et ] sicut PEL var

cognouistis ] cognouisti 54 * $\mathrm{PEL}^{\mathrm{B}}$, cognoscitis $61 \mathrm{PEL}^{\text {var }}$

nos ex parte ] nos ex uel a parte 77 , non ex parte $\mathrm{AMst}^{\mathrm{var}}$, ex parte nos PEL var quia ] om. 54, qua 58 , quod PEL var

gloria uestra ] + nos 58 PELvar

sumus sicut et uos nostra ] sumus sicut et uos $5^{8}$

in die ] in diem $61647576 \mathrm{PEL}^{\mathrm{B}}$

domini nostri iesu $\mathrm{AMst}^{\mathrm{R}}$ ] domini iesu $7576 \mathrm{AMst}^{\mathrm{ed}} \mathrm{PEL}^{\text {var }}$

christi ] om. AMst ${ }^{\mathrm{A}}$

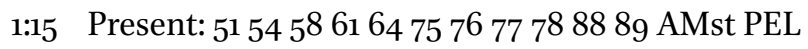

et hac ] et haec 757689 AMst $^{\text {var }}$ PEL $^{\text {var }}$

confidentia 5488 ] fiducia (64) AMst

uolui prius ] prius uolui 61, uolueram primum 64, uolueram prius 757689

AMst $\mathrm{PEL}^{\mathrm{B}}$, uolui uel cogitaui prius 77, uolueram PEL var

uenire ad uos ] + et a uobis praemitti in iudaeam $77^{\dagger}+$ prius PEL var

ut ] et 89

secundam ] secundum $61^{*} 757689 \mathrm{AMst}^{\mathrm{var}} \mathrm{PEL}^{\mathrm{B}}$, plenam $\mathrm{AMst}^{\mathrm{R}}$

gratiam haberetis $\langle 64\rangle$

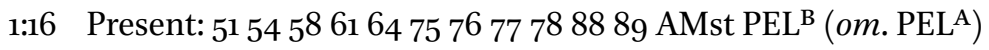

et per uos

transire in macedoniam $\mathrm{AMst}^{\mathrm{A}(\mathrm{R})} \mathrm{PEL}^{\mathrm{var}}$ ] transirem in macedoniam 61 AMst $^{\text {ed }} P E L^{B}$, proficisci in macedoniam 64757689 , proficerer uel transirem in macedoniam 77 , macedoniam proficisci PEL ${ }^{\text {var }}$

et iterum a macedonia 61 (64) 7589 ] et iterum macedonia AMst ${ }^{\text {var }}$ uenire $\mathrm{AMst}^{\text {var }} \mathrm{PEL}^{\text {var }}$ ] uenirem $5461 \mathrm{AMst}^{\mathrm{ed}} \mathrm{PEL}^{\mathrm{B}}$, transire PEL var

$\dagger{ }_{2}$ Cor. 1:15. The Greek text underneath this addition in VL 77 has been deleted (cf. 2 Cor, 1:16). 
ad uos et a uobis

deduci ] praemitti $6475767789 \mathrm{PEL}^{\text {var }}$, duci $88^{\mathrm{C}}$

in iudaeam 515458617888 ] in iudaea 89 , in eadem AMst ${ }^{\text {var }}$

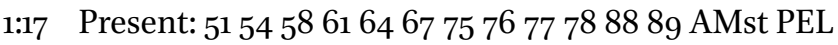

cum hoc ergo ] cum ergo hoc $5_{1} \mathrm{PEL}^{\text {var }}$, hoc ergo 6477 AMst, cum ergo 75 76, cum autem hoc PEL var

uoluissem ] cogitassem $61757689 \mathrm{PEL}^{\mathrm{B}}$, uolens 64, cogitans $77 \mathrm{AMst}$; + hoc 7576

numquid 5477 ] + igitur 77

leuitate usus sum ] leuitate usum sum 6r, leuitatem usus sum 647576

aut ] an 51 ; + numquid AMst $^{\mathrm{R}}$

quae cogito $51545861\langle 67\rangle$ ] quae cogitatio 77

secundum carnem ] lac. 67

cogito ] cogitatio 77

ut sit ] ut scit $54^{*}$, ut sic PEL ${ }^{\mathrm{B}}$, ut si PEL ${ }^{\text {var }}$

apud me 51586475

est ] etiam etiam 64, est est $7576 \mathrm{PEL}^{\mathrm{A}}$, utique uel est utique uel est $77, \mathrm{om}$.

AMst ${ }^{\text {tar }}$

et $\mathrm{AMst}^{\mathrm{var}}$ ] om. $64 \mathrm{PEL}^{\mathrm{var}}$, est AMst ${ }^{\mathrm{ed}}$

non AMst ${ }^{\text {var }}$ ] non non 64757677 AMst $^{\text {ed }} \mathrm{PEL}^{\mathrm{A}}$, non est 89

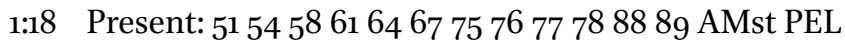

fidelis autem ] fidelis PEL ${ }^{\text {var }}$, fidelis est PEL ${ }^{\text {var }}$

deus ] deus est 51 , om. PEL ${ }^{\text {A* }}$

quia sermo noster ] quia sermo meus $5^{8}$

qui ] om. AMst

fit ] sit 5178 , fuit $54^{\mathrm{C}} 88^{*} \mathrm{PEL}^{\mathrm{var}}$, om. 64 AMst, lac. 67

apud $51545875\langle 76\rangle \mathrm{AMst}^{\mathrm{R}}$ ] ad $64777888 \mathrm{PEL}^{\mathrm{var}}$, lac. 67, om. AMst ${ }^{\mathrm{ed}}$

uos $\left.\mathrm{AMst}^{\mathrm{R}}\right]$ nos 51, lac. 76 , om. AMst ${ }^{\mathrm{ed}}$

non est ] non fuit $89 \mathrm{AMst}^{\mathrm{R}}$

in illo ] om. 6475767789 AMst

est ] om. 54, etiam 64, est uel utique 77

et non ] om. $54^{*}$

\pm sed est in illo est $5^{1} 54^{\mathrm{C}} 5^{8} 617^{8} \mathrm{AMst}^{\mathrm{R}} \mathrm{PEL}^{\mathrm{B}}$, sed est in illo est et non $54^{*}$, sed est in illo $88^{*}$

1:19 Present: $3151545^{8} 616467757677788889$ AMst PEL

dei enim filius ] fratres dei enim filius 31, lac. 67

iesus christus (75) ] christus 31, om. PEL ${ }^{\text {var }}$, lac. 67 
qui in ] lac. 67 , in 78

uobis ] + est qui $6175767789 \mathrm{AMst}^{\mathrm{PEL}}{ }^{\mathrm{B}}$, + est PEL var

per nos praedicatus est $51545^{8}\langle 67\rangle 7_{6} 88^{\mathrm{C}}$ ] per nos praedicatur $61 \mathrm{AMst}^{\mathrm{var}}$, per nos praedicatus $7788^{*} \mathrm{PEL}^{\text {var }}$

per me et ] om. PEL $^{\mathrm{A}}$

siluanum et timotheum 5154586164677576 ] saluanum et timotheum $78^{*}$, om. PELA

non fuit ] fuit $89 ;+$ in illo $5154^{\mathrm{C}} 5^{8} 61 \mathrm{AMst}^{\mathrm{R}}$, + in eo PEL var

est et non sed ] etiam et non sed $64,0 m$. PEL var

est ] etiam 64, est uel utique 77, om. $78^{*}$, fuit PEL var

in illo fuit ] fuit in illo 64, illo fuit 89, in illo est PEL var

1:20 Present: $3151545^{8} 6164677576777888$ 89 AMst PEL

quotquot ] quicquid 31 , quodquod 647589

enim ] autem 61, om. PEL ${ }^{\text {var }}$

promissiones dei sunt $\left.54^{*}\left(5^{*}\right)\right]$ sunt promissiones dei 64 , promissiones

dei $89 \mathrm{PEL}^{\mathrm{B}}$, promissionis sunt $P E L^{\text {var }}$

in illo

est ] etiam 64, est uel utique 77 , est etiam $\mathrm{PEL}^{\mathrm{B}}$, id est uerum est PEL var

ideo (77) $\mathrm{AMst}^{\mathrm{D}}$ ] [ $p$ ]ropterea 64, om. $757689 \mathrm{AMst}^{\mathrm{ed}} \mathrm{PEL}^{\mathrm{B}}$, ut $\mathrm{AMst}^{\mathrm{var}}$

et ] lac. 64, om. $88^{*} \mathrm{AMst}^{\mathrm{var}} \mathrm{PEL}^{\mathrm{B}}$

per ipsum $\mathrm{AMst}^{\mathrm{R}}$ ] per eum 64, per [...] 67, in illo AMsted, in illo per ipsum

$\mathrm{PEL}^{\text {var; }}+$ et in illo $\mathrm{AMst}^{\mathrm{D}}$

amen ] lac. 67 , amen uel uere 77 , gratiarum actio amen PEL ${ }^{\mathrm{var}}$

deo ] dico $51 ;+$ dicimus $54^{\mathrm{C}}$

ad ] et 31 , per $61^{*}$

gloriam $\langle 64\rangle 7^{8}$ ] honorem $7^{8 \mathrm{gl}}$; + et honorem 77

nostram 78 ] ad nos $61^{*}$, per nos $61^{\mathrm{C}} 757^{6} 777^{\text {gl }} 89$ AMst PEL $L^{\mathrm{B}}$, lac. 64

1:21 Present: $3151545^{8} 616467757677788889$ AMst PEL SPM

qui autem confirmat nos ] lac. 64 , qui autem con[...] 67, qui autem confirmauit nos PEL ${ }^{\mathrm{A}}$

uobiscum ] [2] biscum 67, nobiscum uel uobiscum 77 , nobiscum 78 SPM $^{\text {var; }}$ + sum PEL ${ }^{\text {var }}$

in christum ] in christo $3^{1} 5^{1} 545^{8} 647576777^{8} 88 \mathrm{PEL}^{\mathrm{var}} \mathrm{SPM}^{\mathrm{var}}$, christus dominus $6189 \mathrm{AMst}^{\text {ed }} \mathrm{PEL}^{\mathrm{B}} \mathrm{SPM}^{\mathrm{ed}}$, in christ[...] 67, christus dominus est $\mathrm{AMst}^{\mathrm{R}}$, deus $\mathrm{SPM}^{\mathrm{var}}$

et qui unxit 64 ] et coniunxit 31, [...] unxit 67

nos deus ] nos $5158 \mathrm{SPM}^{\mathrm{var}}$, nos christus $\mathrm{SPM}^{\mathrm{var}}$ 
1:22 Present: 315154586164677576777888 89 AMst PEL SPM et qui AMstrar ] et $6164 \mathrm{SPM}^{\text {var }}$, lac. 67 , qui et AMsted signauit nos et dedit $\langle 67\rangle]$ signauit nos dedit PEL ${ }^{\mathrm{var}}$; + nobis $\mathrm{SPM}^{\mathrm{var}}$ pignus ] lac. 67 spiritus ] spiritus sui 31 , spiritu[1] 51, spiritum 6489 AMst SPM ${ }^{\mathrm{var}}$, christi PEL var

in cordibus AMst $\left.{ }^{\mathrm{var}}\right]$ cordibus AMst ${ }^{\mathrm{ed}}$ nostris ] uestris $64^{\mathrm{C}}$; + deus est $5^{8}$

1:23 Present: 5154586164677576777888 89 AMst PEL CAr ego autem testem ] ego uero testem AMst ${ }^{\text {ed }}$, ego uere testem AMst ${ }^{\text {var }}$ deum ] lac. 67, om. 88*, domini AMst ${ }^{\mathrm{var}} \mathrm{PEL}^{\mathrm{B}}$, deo PEL var inuoco ] in uoce $\mathrm{PEL}^{\mathrm{B}}$ in $\mathrm{AMst}^{\mathrm{var}}$ ] super $64 \mathrm{AMst}^{\mathrm{ed}}$, et $\mathrm{PEL}^{\mathrm{B}}$ animam meam ] meam animam 757677 quod parcens uobis ] quoniam parcens uobis 64 , abs. CAr non ] nondum $64 \mathrm{AMst}^{\mathrm{R}}$, abs. CAr ueni $\left(54^{*}\right)$ ] lac. 67 , uenio 7576 , abs. CAr ultra corinthum ] corinthum 616475767789 AMst PEL $^{\mathrm{A}}$, lac. 67, abs. CAr

1:24 Present: 5154586164677576777888 89 AMst PEL

non quia ] non quod AMst; + q[3] 67 dominamur ] dominantur 78 , dominemur AMst fidei uestrae $51545^{8}$ ] lac. 67 sed ] sed quod AMsted, sed quod et AMst ${ }^{\text {var }}$ adiutores ] cooperatores 64 , cooperarii $\mathrm{PEL}^{\mathrm{B}}$ sumus ] simus $88^{*} \mathrm{AMst}^{\mathrm{var}}$; + in christo iesu et cooperatores $5^{8}$ gaudii uestri 88 ] gratiae uestrae 6189 , uestrae gloriae $P E L^{B}$ nam fide $\mathrm{AMst}^{\mathrm{R}}$ ] fide enim $64 \mathrm{AMst}^{\text {ed }}$ stetistis ] statis 51545861647576777889 AMst PEL $^{\mathrm{B}}$

2:1 Present: 5154586164677576777888 89 AMst PEL statui ] iudicaui uel statui 77 , decreui AMst, iudicaui PEL ${ }^{B}$ autem ] enim 64, om. 88* PEL var hoc ipse ] hoc ipsum $5^{1} 54^{\mathrm{C}} 5^{8} 757^{6} 777^{8} 88^{\mathrm{C}} 89 \mathrm{PEL}^{\mathrm{A}}$, hoc 6164 AMst, om. $88^{*} ;+$ ut 89 apud me 5154586475 ] lac. 67 , apud 78 ; + ipsum 61, + hoc ipsum 77 ne iterum ] ut non 78 in tristitia uenirem $\left.51547888 \mathrm{PEL}^{\mathrm{var}}\right]$ in tristitiam ueniam 64 , in [...] 67, in tristitiam uenirem $(75)(76) 89 \mathrm{PEL}^{\mathrm{A}}$, uenirem in tristitiam $\mathrm{PEL}^{\mathrm{B}}$ ad uos ] lac. 67 


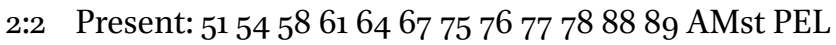

si enim ] nam si 64

ego contristo AMst ${ }^{\mathrm{var}}$ ] ego contristor $5^{8} 61$, contristo AMst ${ }^{\text {ed }}$, contristo ego

PEL ${ }^{\mathrm{A}}$

uos et quis est ] uos quis est 51 PEL var

qui me laetificet 51545888 ] qui me laetificat 617576 89, [qui me] laetifi[3]

67 , qui laetificat me 77

nisi qui ] nisi quis $75 \mathrm{PEL}^{\mathrm{B}}$

contristatur ] contristatus est $\mathrm{AMst}^{\mathrm{var}}$, contristat PEL ${ }^{\mathrm{var}}$

ex me ] lac. 67

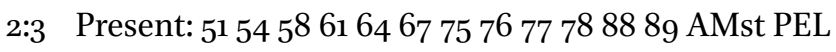

et $\mathrm{AMst}^{\mathrm{R}}$ ] lac. 67 , ideo et AMst ${ }^{\mathrm{ed}}$

hoc ipsum scripsi ] lac. 67, hoc ipsud scripsi 89 *, scripsi hoc ipsum AMst ${ }^{\text {ed }}$, scripsi uobis hoc ipsum AMst ${ }^{\mathrm{R}}$; + uobis $5^{1} 545^{8} 6164757677788889$ PEL $^{\mathrm{B}}$

ut non ] ut 54, ne 64 AMst

cum uenero $\langle 64\rangle]$ ueniens uel cum uenero 77 , cum uenissem AMst; + non $54^{\mathrm{C}}$

tristitiam 5154

super tristitiam habeam 51 (78) 88 ] habeam super tristitiam 54 , habeam $5^{8}$

64, haberem AMst; + ex his 64

de quibus $\mathrm{AMst}^{\mathrm{R}}$ ] a quibus $6164757689 \mathrm{AMst}^{\mathrm{ed}}$

oportuerat ] oporteat 61 , oportebat 64 , oportueram 89

me gaudere confidens in omnibus uobis $\left(76^{*}\right)$ ] me gaudere certum habens AMst

quia ] lac. 67 , quia uel quoniam 77 , qui $\mathrm{PEL}^{\mathrm{A} *}$

meum ] mecum 78

gaudium omnium uestrum est ] gaudium omnium uestrum AMst ${ }^{\text {var }}$

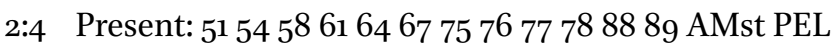

nam ex multa ] nam ex uestra 7576

tribulatione et angustia cordis 54] pressura et angustia cordis AMst PEL var(ed)

scripsi uobis per 64 ] scripsi uobis 67

multas lacrimas ] lacrimas multas $61^{*}$

non ut ] ut non $5^{1} 5^{867}$ AMst $^{\text {var }}$

contristemini sed ut $\langle 67\rangle]$ tristemini sed ut PEL var

sciatis ] sciatis dilectionem 64, caritatem sciatis 757689 , sciatis caritatem

77 , cognoscatis caritatem AMst ${ }^{\mathrm{ed}}$, cognoscatis $\mathrm{AMst}^{\mathrm{R}}$ 
quam $\langle 76\rangle$ ] quia 61 , quod 89

caritatem habeo abundantius (78) ] caritatem habeam abundantius 5154 586188 AMst ${ }^{R}$, habeo abundantius 6475767789 AMsted, abundantius habeam caritatem $P E L^{B}$, abundantius caritatem habeam PEL var(ed) in uobis ] ad uos 6477 , erga uos AMst ${ }^{\mathrm{R}}$

2:5 Present: 5154586164677576777888 89 AMst PEL SPM SPE si quis autem ] si autem quis 77 contristauit ] me contristauit 5158 , contristabit $64 \mathrm{SPE}^{\mathrm{var}}$, contristat uos 89, contristauit me $\mathrm{AMst}^{\mathrm{R}}$, contristabit me $\mathrm{SPE}^{\mathrm{var}}$ non me contristauit ] om. $5^{8} 67$, non me contristabit $64 \mathrm{SPE}^{\mathrm{var}}$ sed ex parte ut non (64)] sed ut non PEL ${ }^{\mathrm{var}}$, ut non $\mathrm{SPM}^{\mathrm{var}}$ onerem $5_{4}^{c} 616788^{c}$ ] honorem $54^{*} 6488^{*} \mathrm{AMst}^{\mathrm{var}} \mathrm{PEL}^{\text {var }}$, grauarem $757^{6}$ 77, orem AMstrar omnes uos AMstvar $]$ uos omnes AMst ${ }^{\text {ed }} \mathrm{PEL}^{\text {var }}$, uos SPM ${ }^{\text {var }}$

2:6 Present: 515458616467757677788889 AMst PEL SPM SPE sufficit $\langle 67\rangle$ ] satis est $64 \mathrm{AMst}^{\mathrm{R}}$, subiecit PEL var illi ] ei $61 \mathrm{AMst}^{\mathrm{ed}} \mathrm{PEL}^{\mathrm{B}} \mathrm{SPM}^{\mathrm{var}}$, om. 64, enim AMst ${ }^{\mathrm{var}}$ qui eiusmodi est ] qui talis est 61 AMst, huiuscemodi 64 obiurgatio haec 51545861 (75) PEL var ] correptio haec 64, correptio uel obiurgatio haec 77 , haec AMst, ista obiurgatio PEL ${ }^{\text {var }}$, haec obiurgatio PEL ${ }^{\text {var }}$, correptio ista $\mathrm{SPM}^{\mathrm{var}}$

quae fit $54^{c} 5^{8} 6188$ ] quae sit 51 , quae fuit $54^{*}$, quae $64 \mathrm{AMst}^{\mathrm{var}}$, qu[...] 67 , om. 77

a pluribus ] a multis 64, lac. 67, om. 77, a plurimis $\mathrm{PEL}^{\text {var }}$; obiurgatio AMsted, + est obiurgatio $\mathrm{AMst}^{\mathrm{A}}$

2:7 Present: 5154586164677576777888 89 AMst PEL SPM SPE ita ut e ] ita ut $757689 \mathrm{AMst}^{\mathrm{var}} \mathrm{PEL}^{\mathrm{var}} \mathrm{SPE}^{\mathrm{var}}$, ita ut hae 88 , ita ut ea SPEvar contra PEL var ] contrario $51545^{8} 61646775767778 \mathrm{PEL}^{\mathrm{AB}} \mathrm{SPM}^{\mathrm{var}} \mathrm{SPE}^{\mathrm{var}}$ magis ] magis uos 64, uos magis 757677

donetis ] doletis AMst ${ }^{\text {var }}$

et ] ut 61

consolemini 61 ] co[...]mini 67 , consolamini AMst ${ }^{\mathrm{var}} \mathrm{PEL}^{\text {var }}$, consolationem PEL ${ }^{\text {var; }}+$ eum 61

ne forte ] ne quomodo 64 AMst

abundantiori tristitia $\left.51545861\langle 67\rangle 88 \mathrm{AMst}^{A \mathrm{R}} \mathrm{SPE}^{\mathrm{var}}\right]$ maiore tristitia 64 (75) $76 \mathrm{PEL}^{\mathrm{A}}$, abundantiore tristitia $77 \mathrm{AMst}^{e d} \mathrm{SPE}^{\mathrm{ed}}$, maiorem tristitiam 89, maiori tristitia SPM 
absorbeatur qui 64

eiusmodi est ] huiusmodi est AMst ${ }^{\mathrm{var}}$, huiuscemodi est SPM${ }^{\mathrm{var}}$

2:8 Present: 5154586164677576777888 89 AMst PEL SPM SPE propter quod obsecro uos $\langle 67\rangle$

ut confirmetis $\langle 67\rangle$ ] confirmetis 64 , confirmare uel ut confirmetis 77

in illum $\mathrm{AMst}^{\mathrm{var}}$ ] in eo 64, illum 7576 , in illo $\mathrm{AMst}^{\mathrm{ed}} \mathrm{SPM}^{\mathrm{var}} \mathrm{SPE}^{\mathrm{var}}$ caritatem ] caritate AMstvar

2:9 Present: $51545^{8} 6164677576777888$ 89 AMst PEL SPM SPE ideo $\langle 67\rangle 75$ ] ad hoc $64 \mathrm{AMst}^{\text {ed }} \mathrm{PEL}^{\mathrm{B}}$, ob hoc uel in hoc $77, \mathrm{om}$. $\mathrm{AMst}^{\mathrm{R}}$ enim ] lac. 67, et enim 76, nam ad AMst ${ }^{\mathrm{R}}$, autem PEL ${ }^{\mathrm{var}}$ et scripsi 54 PEL $^{\text {var }}$ SPM $^{\text {var }}$ ] scripsi 61647689 AMst $^{\mathrm{A}}$ PEL $^{\mathrm{A}}$ SPM $^{\text {ed }}$ SPE, lac.

67 , hoc scripsi AMst ${ }^{\mathrm{R}} \mathrm{PEL}^{\text {var }}$, et hoc scripsi $\mathrm{PEL}^{\mathrm{B}}$ \pm hanc epistulam $54^{\mathrm{C}} 58$, uobis $777889 \mathrm{sPM}^{\text {ed }}$

ut cognoscam $\langle 64\rangle][\ldots]$ am 67 , ut scirem $\mathrm{AMst}^{\mathrm{ed}}$, ut probarem $\mathrm{AMst}^{\mathrm{R}}$, et cognoscebam SPM ${ }^{\mathrm{var}}$

experimentum $\langle 67\rangle$ ] probationem 64, expedimentum 75 76, om. AMst uestrum ] uestram 64, lac. 67, omnium uestrum 77 , om. AMst an ] si $6164757677 \mathrm{PEL}^{\mathrm{B}}$ SPM, lac. 67, quoniam 89 in omnibus ] lac. 67 , in omnia 77 oboedientes sitis $5154^{c} 5^{8}\langle 67\rangle 78$ ] oboedientes estis sitis $54^{*}$, oboedientes estis $6177 \mathrm{PEL}^{\mathrm{B}} \mathrm{SPM}^{\mathrm{ed}}$, obaudientes estis $\langle 64\rangle 757_{6} \mathrm{AMst}^{\mathrm{ed}}$, oboedistis 89 , obaudientes sitis $\mathrm{AMst}^{\mathrm{R}} \mathrm{SPM}^{\mathrm{var}}$

2:10 Present: 5154586164677576777888 89 AMst PEL SPM SPE cui ] si cui $61647788^{\mathrm{C}} 89 \mathrm{AMst} \mathrm{PEL}^{\mathrm{B}} \mathrm{SPM}^{\text {ed }}$, lac. 67 , sicut $757_{6}$ $\mathrm{SPM}^{\mathrm{var}}$

autem aliquid $\langle 67\rangle]$ ali[...] 64, autem quid $\mathrm{AMst}^{\mathrm{R}}$, aliquid autem $\mathrm{AMst}^{\mathrm{var}}$, aliquid $\mathrm{SPE}^{\mathrm{var}}$

donatis PEL ${ }^{\text {var }}$ ] donastis 5158788889 AMst $^{\text {var }}{ }^{2}$ LL $^{\mathrm{AB}}$ SPM $^{\mathrm{var}}$ SPE, lac. 6467 ; + uel donastis 77

et ego $\langle 67\rangle$ ] lac. 64, om. PELvar

nam et ego $\mathrm{AMst}^{\mathrm{R}}$ ] lac. 64, etenim ego $77 \mathrm{AMst}^{\mathrm{ed}}$, etenim AMst ${ }^{\mathrm{var}}$, nam SPM

quod ] quid 61, lac. 64, om. $89 \mathrm{AMst}^{\mathrm{R}}$, quae $P E L^{B}$; + et 77 donaui ] lac. 6467, om. 89 AMst $^{\mathrm{var}} \mathrm{PEL}^{\mathrm{B}}$ SPM; + a me 61 si quid 5461 ] si quod $61^{\text {alt }}$, lac. 6467 , si cui 89 , om. AMst' ${ }^{\mathrm{var}} \mathrm{PEL}^{\mathrm{B}}$ SPM; + et 77 donaui $\left(75^{*}\right)$ ] lac. 6467

propter uos $\langle 67\rangle$ ] lac. 64, per uos $\mathrm{PEL}^{\text {var }}$; + donaui $\mathrm{AMst}^{\mathrm{R}}$ 
in persona ] lac. 64, in faciem 757689 , in faciem uel personam 77 , in facie $\mathrm{PEL}^{\mathrm{B}}$

christi ] lac. 64

2:11 Present: 5154586167757677788889 AMst PEL SPM SPE

ut non ] lac. 67 , ne AMst

circumueniamur $\langle 67\rangle$ ] possideamur uel circumueniamur 77 , possideamur

89 AMst SPM, circumueniamini PEL ${ }^{\text {var }}$

a satana $515^{8}$ ] lac. 67 , a satanan $89 \mathrm{PEL}^{\mathrm{A}} \mathrm{SPM}^{\mathrm{var}} \mathrm{SPE}^{\mathrm{var}}$

non $\mathrm{AMst}^{\mathrm{R}}$ ] lac. 67 , nec $\mathrm{AMst}^{\mathrm{ed}} \mathrm{PEL}^{\mathrm{B}}$

enim ignoramus ] enim $61757677 \mathrm{PEL}^{\mathrm{B}}$, lac. 67, ignoramus enim AMstvar, ignoramus $\mathrm{PEL}^{\mathrm{var}}$

cogitationes eius 78 ] uersutias eius $61757^{6} 7^{8^{\mathrm{gl}}} \mathrm{AMst}^{\mathrm{ed}} \mathrm{PEL}^{\mathrm{B}}$, lac. 67, eius uersutias 77, eius consilia 89, astutias eius AMst ${ }^{\mathrm{var}}$, uersutiam eius $\mathrm{AMst}^{\text {var }}$; + uersutias $58,+$ ignoramus $61757677 \mathrm{PEL}^{\mathrm{B}}$

2:12 Present: 51545861677576777888 89 AMst PEL CAr

cum uenissem autem ] lac. 67, ueniens uel cum uenissem autem 77 , cum autem uenissem AMst

troadem 67 AMst $^{\mathrm{var}}$ ] troade $5158788889 \mathrm{AMst}^{\mathrm{var}} \mathrm{CAr}$, lac. 67 , troadam 75 76 AMst $^{\text {ed }}$, troada 77

propter euangelium christi $545867\langle 67\rangle$ ] propter euangelium 89

et ostium mihi 586177 ] lac. 67 , ostium mihi PEL ${ }^{\text {var }}$, abs. CAr

apertum esset 54 ] apertum $61^{*}$, lac. 67 , esset apertum 77 , apertum est $\mathrm{AMst}^{\mathrm{var}}$ PEL ${ }^{\mathrm{var}}$, abs. CAr

in domino ] abs. CAr

2:13 Present: 51545861677576777888 89 AMst PEL

non ] et non $\mathrm{AMst}^{\mathrm{A} *}$

habui requiem ] lac. 67

spiritui meo AMstvar ${ }^{\mathrm{var}}$ lac. 67 , spiritu meo $757678 \mathrm{PEL}^{\mathrm{var}}$, in spiritu meo AMsted

eo quod non ] quod non PEL var

inuenerim AMst ${ }^{\mathrm{var}}$ ] inuenirem $61757688 * 89 \mathrm{AMst}^{\mathrm{ed}} \mathrm{PEL}^{\mathrm{var}}$, inue[...] 67

titum fratrem meum sed 58 ] [...] sed 67 , tum fratrem meum sed PEL ${ }^{A^{*}}$

ualefaciens eis ] ualedicens eis $61 \mathrm{PEL}^{\text {var(ed) }}$, cum uale illis dixissem $757_{7}$, cum uale illi fecissem 89, uale illis dixi et AMst ${ }^{\text {ed }}$, uale illis facto $\mathrm{AMst}^{\mathrm{R}}$, ualedicens PEL var

profectus sum

in macedoniam $6175^{88^{c}} 89$ ] lac. 67 , in macedonia AMstvar 


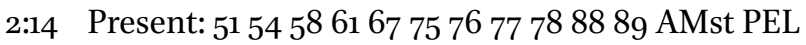

deo autem gratias $\langle 67\rangle]$ ideo autem gratias 54 , deo gratias PEL var qui semper triumphat $61\langle 67\rangle$ ] semper triumphanti $77 ;+$ per $\mathrm{AMst}^{\mathrm{R}}$ nos in christo ] [...] christo 67 iesu AMst ${ }^{\mathrm{DR}} \mathrm{PEL}^{\mathrm{var}}$ ] lac. 67, om. 7576777889 AMst $^{\mathrm{ed}} \mathrm{PEL}^{\mathrm{AB}}$; + domino nostro AMst ${ }^{\mathrm{R}}$

et odorem notitiae suae manifestat 51545888

per nos in omni loco ] lac. 67 , pro nos in omni loco PEL var

2:15 Present: $5^{1} 545^{8} 61677576777888$ 89 AMst PEL

quia christi ] lac. 67 , quia christus 75

bonus odor ] + uel bona flagrantia 77

sumus deo in $(75)]$ sumus [...] 67

his qui salui fiunt $\left.5^{8}\langle 67\rangle\right]$ saluatis uel in saluantibus 77

et in his qui pereunt $5861\left(89^{*}\right)$ ] et in pereuntibus 77

2:16 Present: $5^{1} 545^{8} 61677576777888$ 89 AMst PEL

aliis ] quibusdam $61 \mathrm{PEL}^{\mathrm{B}}$, lac. 67 , et aliis $88^{\mathrm{C}}$, iis $\mathrm{AMst}^{\mathrm{R}}$

quidem ] lac. 67, om. PEL var; + sumus AMst ${ }^{\text {var }}$

odor mortis in mortem $54\langle 67\rangle$ ] odor uitae in uitam 61, odor mortis in morte

AMst ${ }^{\mathrm{var}}$, om. $\mathrm{PEL}^{\mathrm{B}}$

aliis ] illis $\mathrm{AMst}^{\mathrm{R}}$, om. $\mathrm{PEL}^{\mathrm{B}}$, quibusdam PEL var

autem $\langle 67\rangle$ ] quidem 51, om. $\mathrm{PEL}^{\mathrm{B}}$

odor uitae in uitam 51545888 ] odor mortis in mortem 61, lac. 67, odor uitae ad uitam AMst

et ad haec 5154586176 ] lac. 67 , et haec 78 , ad haec PEL var

quis ] qui sunt $\mathrm{AMst}^{\mathrm{var}}$, quis est $\mathrm{PEL}^{\mathrm{B}}$

$\operatorname{tam} \mathrm{AMst}^{\mathrm{AD}}$ ] om. AMst $\mathrm{od}^{\mathrm{ed}}$

idoneus 5158 ] lac. 67; + est 617576 AMst $^{\text {var }}$, + uel sufficiens est 77

2:17 Present: $5^{1} 545^{8} 61677576777888$ 89 AMst PEL

non enim sumus sicut ] lac. 67

plurimi adulterantes $\langle 67\rangle$ ] ceteri uel plurimi adulterantes 77

uerbum dei sed ] lac. $67 ;+$ sicut 89

ex sinceritate 61 ] lac. 67

sed PEL var ] om. $5^{1} 5^{\mathrm{C}} 5^{8} 61757677788889$ AMst PEL ${ }^{\mathrm{B}}$, lac. 67

sicut ex deo ] lac. 67, om. 89

coram deo ] coram ipso $61 \mathrm{PEL}^{\mathrm{B}}$, lac. 67, om. $\mathrm{AMst}^{\mathrm{AD}}$, ipso PEL ${ }^{\text {var }}$

in christo loquimur ] lac. 67 
3:1 Present: 51545861757677788889 AMst PEL

incipimus $\left(88^{*}\right)$ ] aut incipimus 61

iterum nosmet ipsos ] nos iterum 89

commendare ] + uobis 78

aut numquid 5477 ] numquid $\mathrm{AMst}^{\mathrm{R}}$

egemus 89 ] indigemus $6177 \mathrm{AMst}^{\mathrm{PEL}} \mathrm{L}^{\mathrm{B}}$, egimus $88^{*}$

sicut quidam

commendaticiis epistulis 5176777888 ] commendaticias epistulas 617589 $P E L^{B}$

ad uos aut ex uobis $\left.\left(61^{*}\right)\right]$ ad uos aut a uobis $89 \mathrm{PEL}^{\mathrm{B}}$

\pm aliquas 61 , commendaticias 7589 , commendaticiis 76 , commendaticiis epistulis 77 , aliquas commendaticias $\mathrm{PEL}^{\mathrm{B}}$

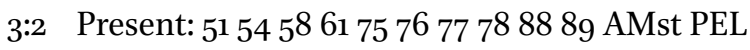

epistula nostra uos estis $5_{1} 6176777888\left(89^{*}\right)$ ] epistula enim nostra uos estis $5^{1}$

scripta in cordibus (88) ] inscripta in cordibus 61757677

nostris ] uestris PEL var

quae scitur 51545888 ] quae cognoscitur $61 \mathrm{AMst}^{\mathrm{ed}} \mathrm{PEL}^{\mathrm{B}}$, scita uel quae scitur 77, om. AMst var

et ] om. AMst ${ }^{\mathrm{var}}$; + quae $61757689 P E L^{B}$, + quia $\mathrm{AMst}^{\mathrm{var}}$

legitur ] relecta uel quae relegitur 77 , cognoscitur $\mathrm{AMst}^{\mathrm{var}}$, agnoscitur $\mathrm{PEL}^{\mathrm{var}}$ ab omnibus hominibus ] ab hominibus 77

3:3 Present: $5^{1} 545^{8} 61757677788889$ AMst PEL

manifestati ] manifestata 5158 89, manifesta 5461 , manifestatio PEL ${ }^{\mathrm{B}}$, manifeste PEL $^{\text {var }}$, manifestatur PEL ${ }^{\text {var }}$

quoniam ] qui AMstvar, quia AMsted, quam $\mathrm{PEL}^{\text {var }}$

epistula estis christi 587888 ] epistula christi estis ${ }_{51}$, estis epistula christi 61

75767789 AMst $P E L^{B}$

ministrata ] manifestata 88

a nobis ] per nos 6189

et scripta ] inscripta $757_{77} \mathrm{PEL}^{\mathrm{B}}$, scripta 89 AMst

non atramento sed ] + in PEL var

spiritu ] + sancto PEL ${ }^{\mathrm{B}}$

dei uiui non in tabulis lapideis sed in tabulis cordis carnalibus

3:4 Present: $51545^{8} 617576777^{8} 88$ 89 AMst PEL

fiduciam ] confidentiam 75767789

autem ] om. $89 \mathrm{PEL}^{\mathrm{B}}$ 
talem habemus $54^{*}$ ] talem ṇụç̣imus $58^{*}$

per christum ad deum ] ad deum per christum 6189 PEL $^{\text {var }}$, per christum ad dominum $\mathrm{AMst}^{\mathrm{var}}$, ad dominum per christum $\mathrm{PEL}^{\mathrm{B}}$

3:5 Present: 5154586167757677788889 AMst PEL

non quod sufficientes ] lac. 67 , non sufficientes AMst ${ }^{\mathrm{A}}$ *

simus AMstrar ] sumus 546175767789 AMst $^{\text {ed }} \mathrm{PEL}^{\mathrm{A*}}$, lac. 67

cogitare aliquid $54^{*}$ ] lac. 67 , aestimare aliquid AMsted, aliquid aestimare AMst $^{\text {var }}$

a nobis $\langle 67\rangle$

quasi ex nobis ] lac. 67, quasi per nos 89, tamquam a nobis sit AMst, om.

PEL $^{\text {var }}+$ + ipsis 61

sed sufficientia ] lac. 67

nostra ex deo

est ] om. 61, lac. 67

3:6 Present: 51545861677576777888 89 AMst PEL

qui et ] lac. 67 , quia et $\mathrm{AMst}^{\mathrm{A}}$

idoneos $5158\left(\mathrm{PEL}^{\mathrm{A}}\right)^{\mathrm{C}}$ ] idoneus $54^{*}$, lac. 67 , sufficientes 757689 PEL ${ }^{\mathrm{var}(\mathrm{ed})}$, sufficientibus PEL var

nos fecit ] nos facit 61 , lac. 67 , fecit nos 77

ministros noui testamenti $\langle 67\rangle]$ ministros 58

non ] [1] on $54^{*}$, lac. 67

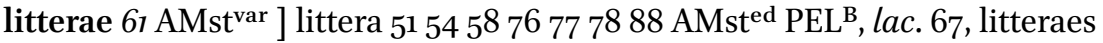
$75^{*}$, litteras $75^{\mathrm{C}}$

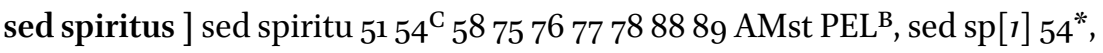
lac. 67

littera enim $6 r\langle 67\rangle]$ nam littera 757689

occidit ] lac. 67

spiritus autem ] spiritus uero 61, lac. 67, spiritu autem 7576

uiuificat $\langle 67\rangle$

3:7 Present: 51545861677576777888 89 AMst PEL CAr

quod si ] si autem 757677

ministratio ] ministerium 757677 PEL $^{\text {var }}$, administratio AMst ${ }^{\text {var }}$

mortis ] om. 51 , litteris mortis 78

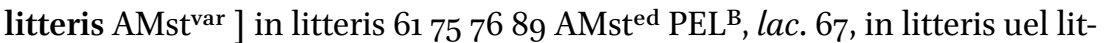
tera 77 , littera CAred, litterae CArvar

deformata ] formata 6175767789 AMst PEL ${ }^{\mathrm{B}} \mathrm{CAr}^{\text {var }}$, [...] ata 67, deformatum PELvar 
in lapidibus ] lapidibus 77 ; + qui AMstvar

fuit $\langle 89\rangle]$ om. PEL var

in gloria ] in gloriam $\mathrm{AMst}^{\mathrm{A}}$

ita ut non ] abs. CAr

possent 61 ] possint $7588 \mathrm{AMst}^{\mathrm{var}} \mathrm{PEL}^{\mathrm{var}(\mathrm{ed})}$, possunt $\mathrm{PEL}^{\mathrm{B}}$, abs. CAr

intendere $\langle 67\rangle$ ] intueri $61 \mathrm{AMst}$, om. $\mathrm{PEL}^{\mathrm{B}}$, abs. CAr; + uel intueri 77

filii israhel $6 r_{75}$ ] lac. 67 , filios israhel $77^{*}$, abs. CAr

in faciem $\langle 67\rangle$ ] abs. CAr

mosi ] moysi $5154^{*} 5861757677788889$ PEL, lac. 67, abs. CAr; + intendere PEL $^{\mathrm{B}}$

propter gloriam ] abs. CAr

uultus eius ] lac. 67, abs. CAr

quae euacuatur $\left.5154^{c} 586188\right]$ q[1] euacuatur $54^{*}$, lac. 67 , quae aboletur

757689 , abolitam uel euacuatam uel quae aboletur 77 , quae euacuabitur PEL ${ }^{\text {var }}$, abs. CAr

3:8 Present: 51545861677576777888 89 AMst PEL

quomodo non magis $\langle 67\rangle$ ] quomodo non 5 , quomodo $P E L^{\text {var }}$

ministratio ] lac. 67, administratio AMst, ministerio PEL ${ }^{\mathrm{var}}$ spiritus

erit in gloria $\mathrm{AMst}^{\mathrm{var}}$ ] erit in gloriam $61 \mathrm{AMst}^{\mathrm{ed}}$, in gloria magis non erit PEL ${ }^{\text {var }}$

3:9 Present: 5154586167757677788889 AMst PEL

nam si ] si enim 6177 AMst PEL $^{\mathrm{B}} ;+$ in $77^{*}$ AMst, + ex 89 PEL $^{\mathrm{B}}$

ministratio $54\langle 67\rangle$ ] ministerio $757677 \mathrm{PEL}^{\mathrm{B}}$, administratione 89 AMst, ministerium PEL var

damnationis $5154^{c} 5861$

gloria est ] in gloria est $545^{8} \mathrm{PEL}^{\mathrm{A} *}$, gloria $\mathrm{AMst}^{\mathrm{ed}}$, est in gloria $\mathrm{AMst}^{\mathrm{D}}$, est gloria $\mathrm{AMst}^{\mathrm{R}}$

multo magis

abundat $5888 \mathrm{AMst}^{\mathrm{R}}$ ] abundet $5^{1} 78 \mathrm{PEL}^{\mathrm{var}}$, abundabit $6177 \mathrm{AMst}^{\mathrm{var}} \mathrm{PEL}^{\mathrm{B}}$, lac. 67, abundauit 757689 AMst ${ }^{\text {ed }}$ PEL $^{\text {var }}$

ministerium $][\ldots]$ is $[\ldots] 67$, ministratio AMst ${ }^{\mathrm{var}}$, administratio $\mathrm{AMst}^{\mathrm{ed}}$

iustitiae $\left.51545^{8} 6778(89)\right]+$ erit 58

in gloria $\left.\mathrm{AMst}^{\mathrm{var}}\right]$ in gloriam $\mathrm{AMst}^{\mathrm{ed}}$, gloria $\mathrm{PEL}^{\mathrm{A}}$

3:10 Present: 51545861677576777888 89 AMst PEL

nam nec ] n[...] 67, nam ne 7576 89, et enim neque 77, nec enim AMst, nam et $P E L^{\mathrm{B}}$, nec ne sic PEL ${ }^{\text {var }}$ 
glorificatum est ] glorificatum $58,[\ldots]$ st 67 , gloriosum factum est AMst, glorificatus est PEL ${ }^{\text {var }}$

quod ] id quod AMst

claruit $\mathrm{AMst}^{\mathrm{R}}$ ] gloriosum factum est $757^{6}$, gloriosum claruit 77 , gloriosum factum 89, glorificatum est AMst ${ }^{\text {ed }}$, glorificatur AMstrar

in hac parte propter

excellentem gloriam ] eam quae supereminet gloriam 7576 (89), super eminentem uel excellentem gloriam 77

3:11 Present: 51545861677576777888 89 AMst PEL

si enim quod ] si autem quod $61 \mathrm{PEL}^{\mathrm{B}}$, si quod PEL var

euacuatur (78) ] abhorrentur 7576 , aboletur 7789

per gloriam est ] per gloriam gloria est 61 , per gloriam 7576 , fuit in gloria $\mathrm{AMst}^{\mathrm{ed}}$, fuit gloria AMst ${ }^{\mathrm{var}}$

multo magis quod manet ] multo magis quod permanet AMst

in gloria ] in gloria gloria 61 , gloria 67 , in gloriam 89

est $\mathrm{AMst}^{\mathrm{var}}$ ] om. $75767789 \mathrm{AMst}^{\mathrm{ed}}$

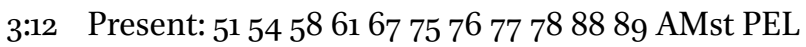

habentes igitur ] habentes ergo 7778 , habentes autem AMstvar

talem spem ] lac. 67, om. PEL var

multa fiducia ] lac. 67 , multa confidentia 7576 PEL ${ }^{\text {var }}$, multam confidentiam

89 ; + uel confidentia 77

utimur AMst ${ }^{\text {var }}$ ] utamur 61757689 AMst $^{\text {ed }}$, utamur uel utemur 77

3:13 Present: $51545^{8} 61677576777888$ 89 AMst PEL

et non sicut $\langle 67\rangle$ ] ut non sicut 89

moses ponebat ] moyses ponebat $5^{1} 54^{*} 5^{8} 617576777^{8} 88$ 89 PEL, lac. 67

uelamen super faciem suam $\langle 67\rangle]$ ] uelamen supra faciem suam $757^{6} 77$

ut non intenderent ] lac. 67 , ad non intendere uel ut non intenderent 77 , ne intuerentur AMst, ut non intenderint PEL var

filii israhel 75$][\ldots] 167$, filiis israhel 77 ; + in faciem moysi $6 r$

in $\mathrm{AMst}^{\mathrm{R}} \mathrm{PEL}^{\mathrm{var}}$ ] usque in $6175767789 \mathrm{PEL}^{\mathrm{A}}$, usque ad $\mathrm{AMst}^{\mathrm{ed}}$ $\mathrm{PEL}^{\mathrm{B}}$

faciem eius $78 \mathrm{AMst}^{\mathrm{R}}$ PEL ${ }^{\text {var }}$ ] finem eius $617576777^{\mathrm{gl}} \mathrm{AMst}^{\mathrm{ed}} \mathrm{PEL}^{\mathrm{AB}}$; + gloriam 77

quod ] lac. 67 , quae 78

euacuatur ] lac. 67, aboletur 757677 (89) 
3:14 Present: 51545861677576777888 89 AMst PEL TES

sed ] lac. 67 , sed si 78 , om. TES

obtusi $\left\langle 54^{\mathrm{C}}\right\rangle 77^{*} \mathrm{AMst}^{\mathrm{R}}$ ] obtunsi $\left\langle 54^{*}\right\rangle 6 r\langle 67\rangle{ }_{75} 7^{6} 7_{77^{\mathrm{C}}} 788889$ AMst $^{\text {ed }}$ PEL $^{\mathrm{A}}$ sunt sensus eorum ] sunt PEL ${ }^{\text {var }}$

usque in hodiernum $\left.54\langle 67\rangle 78^{*} 8889\right]$ usque in hunc TES

enim diem $\langle 67\rangle$ ] diem 546175768889 AMst PEL Tes, diem enim 58

id ipsum uelamen ] sed ipsud uelamen 89, haec ipsa obtusior $\mathrm{AMst}^{\mathrm{R}}$, hoc eodem uelamento TES

in lectione ueteris testamenti $\langle 67\rangle 88$ manet $]$ om. 78 , manente TES; + quod 61 PEL $^{\mathrm{B}}$ TES, + dum 757689 AMsted $^{\text {ed }}+$ et dum AMst ${ }^{\mathrm{var}}$

non reuelatum ] non reuelatur $61757689 \mathrm{AMst}^{\mathrm{PEL}} \mathrm{L}^{\mathrm{B}} \mathrm{TES}$; + uel quod non reuelatur 77

quoniam in christo ] quia in christo $\left(7^{*}\right)^{\mathrm{C}}$ AMst TES

euacuatur ] aboletur 757689 , aboletur uel euacuatur 77

3:15 Present: 5154586167757677788889 AMst PEL tES

sed usque in ] nam usque in 61, et usque in TES

hodiernum diem 5488 ] hodiernum 75767789 TES

cum ] quotiens 617778 , quotienscumque $\mathrm{PEL}^{\mathrm{B}}$, si quando TES

legitur moses ] legitur moyses $5^{1} 545^{8} 61677576777888$ 89 PEL ${ }^{\mathrm{A} B}$ TES, legitur in moysen PEL ${ }^{\text {var }}$, moyses legitur PEL var

uelamen $\mathrm{TES}^{\mathrm{var}}$ ] uelamentum $\mathrm{TES}^{\text {ed }}$

est positum $\mathrm{AMst}^{\mathrm{R}}$ ] positum est 5861 , est positus 78 , est $89 \mathrm{AMst}^{\mathrm{ed}} \mathrm{TES}$, om.

$\mathrm{AMst}^{\mathrm{var}} \mathrm{PEL}^{\mathrm{var}}$; + ignorantiae $P E L^{B}$

super cor eorum AMst ${ }^{\mathrm{var}}$ ] supra cor eorum $5^{1} \mathrm{AMst}^{\mathrm{ed}}$, om. PEL var

3:16 Present: 51545861677576777888 89 AMst PEL TES

cum ] lac. 67 , mox TES

autem 78 ] uero $61777^{\mathrm{gl}} \mathrm{PEL}^{\mathrm{B}}$, lac. 67, om. PEL ${ }^{\mathrm{var}}$

conuersus fuerit $\langle 67\rangle \mathrm{TES}^{\mathrm{var}}$ ] conuersi fuerint $\mathrm{AMst}^{\mathrm{R}}$, conuersus est TES $\mathrm{S}^{\mathrm{ed}}$, conuersus TES ${ }^{\text {var }}$, conuersi es TES ${ }^{\text {var }}$; quis $61,+$ iudaeus PEL ${ }^{\text {var }}$

ad deum AMst ${ }^{\text {var }}$ PEL $^{\text {var }}$ ] ad dominum $5861677788^{*} 89$ AMst $^{\text {ed }}$ PEL ${ }^{A B}$ TES aufertur PEL ${ }^{\text {var }}$ ] auferetur $5^{1} 5^{8} 617576777^{8} 8889$ AMst PEL $^{\text {AB }}$ TES $^{\text {var, }}$, aufer[...] 67 , aufer TES ${ }^{\text {var }}$

uelamen $\langle 67\rangle]$ tegumentum TEs var $^{\text {+ }}+$ ab eis $5^{8}$

3:17 Present: 5154586164677576777888 89 AMst PEL SPM

dominus autem $\mathrm{SPM}^{\mathrm{var}}$ ] deus autem 51 , lac. 64 , nam dominus $89 \mathrm{SPM}^{\mathrm{ed}}$, dominus PEL ${ }^{\mathrm{B}}$, deus enim SPM ${ }^{\mathrm{var}}$,

spiritus est ubi ] lac. 64 
autem ] om. SPM ${ }^{\mathrm{var}}$, lac. 64

spiritus domini ] spiritus $\mathrm{d}[\ldots] 67 ;+$ est 58

ibi libertas $\langle 67\rangle]$ libertas 64 ; + est 61 AMst $^{\text {var }}$ PEL $^{\text {var }}$

3:18 Present: $5^{1} 545^{8} 6164677576777888$ 89 AMst PEL SPM

nos ] non $88^{*}$

uero ] itaque $61 \mathrm{AMst}^{\mathrm{R}} \mathrm{PEL}^{\mathrm{B}}$, autem $75767778 \mathrm{AMst}^{\mathrm{ed}} \mathrm{PEL}^{\text {var }}$, ergo $\mathrm{PEL}^{\text {var }}$

omnes $\mathrm{SPM}^{\mathrm{var}}$ ] om. $88^{\mathrm{C}} \mathrm{AMst}^{\mathrm{var}} \mathrm{SPM}^{\mathrm{ed}}$

reuelata facie gloriam $(64)\langle 67\rangle 7889$

domini ] dei $61 \mathrm{AMst}^{\mathrm{var}} \mathrm{SPM}^{\mathrm{var}}$

speculantes ] spectantes 61 , contemplantes 78 , speculamur PEL var, speculantur PEL var

in ] ad 61 AMst PEL ${ }^{\mathrm{B}}$, om. 757677

eandem SPM ${ }^{\text {var }}$ ] eadem $6489 \mathrm{SPM}^{\text {ed }}$

imaginem $\left.51\left(54^{*}\right) 58 \mathrm{SPM}^{\mathrm{var}}\right] \operatorname{im}[1]$ ginem $89^{*}$, imagine $\mathrm{SPM}^{\mathrm{ed}}$

transformamur $\mathrm{AMst}^{\mathrm{R}}$ ] reformamur $6177 \mathrm{AMst}^{\mathrm{ed}} \mathrm{PEL}^{\mathrm{B}}$, reformarum 75 76, transformatur PEL var, om. PEL var

a claritate in claritatem (88) ] a gloria in gloriam 616477 (89) $\mathrm{AMst}^{\mathrm{ed}} \mathrm{PEL}^{\mathrm{B}}$ SPM, de gloria in gloriam 7576 , in gloriam AMst ${ }^{\text {var }}$ + et a gloria in gloriam 58

tamquam 7677 ] sicut $61 \mathrm{AMst}^{\mathrm{PEL}} \mathrm{B}^{\mathrm{B}}$

a ] de PELvar, om. SPM ${ }^{\text {var }}$

domini spiritu (78) ] domino in spiritu 64

4:1 Present: $5^{1} 54586164677576777888$ 89 AMst PEL SPM SPE

ideo ] propter quod 64, propterea 89 , quare AMst, om. PEL ${ }^{B}$, et ideo SPM

habentes ] + autem $\mathrm{AMst}^{\text {var }} \mathrm{PEL}^{\mathrm{B}}$

hanc ministrationem 54 ] ministrationem hanc $\langle 64\rangle 757677$, hanc administrationem $89 \mathrm{AMst}^{\mathrm{var}} P E L^{B} \mathrm{SPE}^{\mathrm{var}}$

iuxta quod ] sicut $6164757677 \mathrm{PEL}^{\mathrm{B}} \mathrm{SPM}^{\mathrm{var}}$, prout $89 \mathrm{AMst}^{\mathrm{SPM}}{ }^{\mathrm{ed}}$, iuxta PELvar

misericordiam ] misericordia 89

consecuti sumus ] consecutionem 64, consecuti 757677

non deficimus ] non deficiamus $61777889 \mathrm{AMst}^{\mathrm{var}} \mathrm{PEL}^{\mathrm{var}} \mathrm{SPM}^{\mathrm{var}}$, [i]nfirmemur 64, non defecimus $757688 \mathrm{SPE}^{\mathrm{var}}$

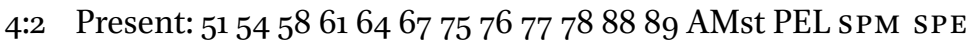

sed abdicamus ] sed abiciamus $64 \mathrm{SPM}^{\mathrm{var}}$, sed renuntiauimus 7576 , sed abiciamus uel renuntiemus 77 , sed abdicemus 78, sed renuntiamus AMst ${ }^{\text {ed }}$, sed renuntiemus AMst ${ }^{\mathrm{var}}$, sed abicimus PEL ${ }^{\mathrm{B}}$, sed abnegamus $\mathrm{PM}^{\text {ed }}$ 
occulta 58 AMst $^{\text {var }}$ ] occultis 7576 AMsted $^{\text {ed }}$

dedecoris ] tenebrarum $58 \mathrm{SPM}^{\mathrm{var}}$, confusionis 64 , pudoris 7576 , dedecoris uel pudoris 77 , uerecundiae 89 , uitiorum dedecoris PEL ${ }^{\text {var }}$ non ambulantes in astutia neque 54$]+$ dolo $64 \mathrm{SPM}^{\mathrm{var}}$ adulterantes uerbum dei ] domini PELA, om. PEL var sed in ] sed 75767789 PEL $^{\text {var }}$ manifestatione 54 ] manifestationem 6489 , manifestatio 7576 ueritatis commendantes 54 nosmet ipsos ] uosmet ipsos SPEvar ad omnem conscientiam hominum 5478 (88) coram deo ] in conspectu dei $64 \mathrm{SPM}^{\mathrm{var}}$

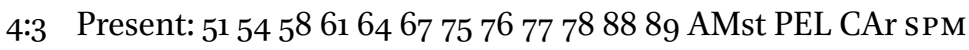
quod si etiam ] quod etiam si 51, quod si 61, si autem et 6477 , sed et si AMsted, quod si adhuc $\mathrm{AMst}^{\mathrm{R}}$, sed si $\mathrm{AMst}^{\mathrm{var}} \mathrm{PEL}^{\mathrm{B}}$, et si $\mathrm{AMst}^{\mathrm{var}} \mathrm{SPM}^{\mathrm{var}}$, sed et PELvar

opertum est $\left.\left(89^{*}\right)\right]$ opertum $5^{1}$, coopertum est $5^{8} 76$, ob[ue]latum est 64 , est opertum uel uelatum 77 , est opertum $\mathrm{PEL}^{\mathrm{B}}$, operatum est PEL var euangelium nostrum in 54586178

his qui pereunt $5861 \mathrm{SPM}^{\mathrm{var}}$ ] pereuntibus 77 , his quae pereunt $89 \mathrm{SPM}^{\mathrm{ed}}$, his est quae pereunt AMstvar

est opertum AMst ${ }^{\text {var }} \mathrm{PEL}^{\mathrm{var}}$ ] opertum est $58618889 \mathrm{SPM}^{\text {ed }}$, est obuelatum 64 , est coopertum 7576, om. AMsted ${ }^{\text {ed }} L^{A B}$ SPM $^{\text {var }}$

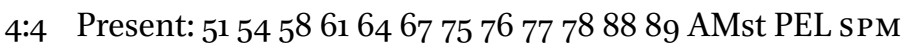
in quibus deus ] in quibus AMstrar, deus PEL var huius saeculi $51545861 \mathrm{AMst}^{\mathrm{var}}$ ] saeculi huius $6475767789 \mathrm{AMst}^{\mathrm{ed}}$ excaecauit $51545^{8} 767788 \mathrm{AMst}^{\mathrm{var}}$ ] excaecabit 64 , et caecauit 78 , obcaecauit $\mathrm{AMst}^{\mathrm{ed}}$, caecauit $\mathrm{PEL}^{\text {var }}$, excitauit $\mathrm{PEL}^{\text {var }}$ mentes $\mathrm{AMst}^{\mathrm{A}}$ ] sensus uel mentes 77 , sensus $\mathrm{AMst}^{\mathrm{ed}} \mathrm{PEL}^{\mathrm{B}}$ infidelium ut non $\langle 64\rangle$ ] infidelium in uel ut non 77 fulgeat ] peruiderent $61 \mathrm{PEL}^{\mathrm{B}}$, uideat non fulgendo 77 , fulgeant 89 , peruideant AMst, uiderant PEL ${ }^{\text {var }}+$ + in eis $58,+$ illis SPM

inluminatio $5158\langle 64\rangle 7678$ ] lumen $61 \mathrm{AMst}^{\mathrm{P} E L^{\mathrm{B}}}{ }^{\mathrm{B}}$, inluminationem 77 , inluminatione 89 euangelii gloriae ${ }_{51}(54) 5861\langle 64\rangle 7788$ ] euangelii maiestatis $\mathrm{AMst}^{\mathrm{R}}$ christi qui est imago 515458 ] christi quae est imago 88 dei ] dei inuisibilis $5154^{\mathrm{C}} 58$, inuisibilis dei $61 \mathrm{PEL}^{\mathrm{B}} \mathrm{SPM}$ 
4:5 Present: $5^{1} 545^{86164677576777888 ~ 89}$ 271 AMst PEL sPE

non enim AMst ${ }^{\mathrm{var}}$ ] nos enim $58^{*}$, fratres non 271, nec enim AMst ${ }^{\text {ed }}$

nosmet ipsos $\langle 64\rangle]$ nos ipsos $\mathrm{AMst}^{\mathrm{R}}$

praedicamus sed $51545^{8} 777888271$ ] sed commendamus $61271^{\text {alt }}$, sed praedicauimus PELvar

iesum christum dominum $\left.\langle 64\rangle \mathrm{AMst}^{\text {var }} \mathrm{PEL}^{\text {var }}\right]$ christum iesum dominum 6189 AMst $^{\text {ed }} \mathrm{PEL}^{\mathrm{AB}}$, dominum iesum iesum 77 , iesum dominum PEL ${ }^{\text {var; }}$ + nostrum $5^{1} 545^{8} 618889271$ AMst PEL ${ }^{\mathrm{AB}} \mathrm{SPE}^{\mathrm{var}}$

nos autem $\langle 64\rangle]$ nos uero $\mathrm{PEL}^{\mathrm{A}}$, nos $\mathrm{SPE}^{\mathrm{var}}$

seruos ] conseruos 271

uestros ] nostros 7576

per ] propter $61 \mathrm{AMst}^{\mathrm{AEL}} \mathrm{B}^{\mathrm{B}}$

iesum ] christum 51 271, christum iesum 61, iesum christum 89

4:6 Present: $5^{1} 545^{861646775767778} 8488$ 89 271 AMst PEL

quoniam ] quia 64 AMst, fratres 84

deus qui ] qui $5188^{*} \mathrm{PEL}^{\mathrm{B}}$

dixit ] eduxit $61 ;+$ lumen splendescere 61

de tenebris $\left\langle\left(64^{*}\right)^{\mathrm{C}}\right\rangle$ ] ex uel de tenebris 77 , om. $271^{*}$

lucem ] lumen $546475767784888921^{\mathrm{C}}$ AMst PEL ${ }^{\mathrm{B}}$, om. $61271^{*}$

splendescere ] om. $61271^{*}$, clarescere 64 , fulgere 757689 AMst, splendere uel fulgere $77, a b s .84$, splendere PEL var

qui ] om. $5^{1} 5^{861} 647576777889271$ AMst PEL ${ }^{\mathrm{B}}$, abs. 84

inluxit $5154^{c} 58767778$ ] induxit $54^{*}$, claruit 64 , abs. 84 , om. $271^{*}$

in cordibus nostris $\langle 64\rangle]$ abs. 84 , cordibus nostris $\mathrm{PEL}^{\mathrm{B}}$

ad inluminationem $5^{1} 54^{c} 5^{8} 767778(89)$ ] ad [3] luminationem $54^{*}$, abs. 84 scientiae 515458271 ] abs. 84, cognitionis AMst ${ }^{\mathrm{ed}}$, cogitationis $\mathrm{AMst}^{\mathrm{var}}$ claritatis ] gloriae 6475767789 AMst, abs. 84

dei $\mathrm{AMst}^{\mathrm{R}}$ ] om. 61, eius $6475767789 \mathrm{PEL}^{\mathrm{var}}$, suae $\mathrm{AMst}^{\mathrm{ed}}$, abs. 84; + quae est 58

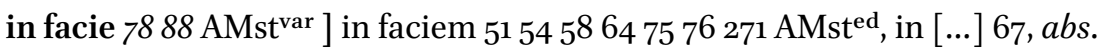
84

christi iesu $\langle 67\rangle]$ abs. 84, iesu christi 88271 PEL $^{\text {var }}$

4:7 Present: 515458616467757677788889 271 AMst PEL sPE

habemus autem $271 \mathrm{AMst}^{\mathrm{var}}$ ] habentes autem AMst ${ }^{\text {ed }}$

thesaurum istum 5461 (64) $271 \mathrm{AMst}^{\mathrm{var}}$ ] thesaurum hunc $75 \quad 7677$ AMst $^{\text {ed }}$

in uasis fictilibus ut $61^{c} 64$ ] in uasibus fictilibus ut $61^{*}$, in fictilibus uasis ut 
sublimitas ] excellentia $61 \mathrm{AMst}^{\text {ed }} \mathrm{PEL}^{\mathrm{B}}$, eminentia $64 \mathrm{AMst}^{\mathrm{R}}$, excellentia uel sublimitas 77

sit uirtutis dei PEL ${ }^{\text {var }}$ ] uirtutis sit dei $5^{1} 64757677 \mathrm{PEL}^{\text {var }}$, sit uirtus dei 61 $271^{*} \mathrm{AMst}^{\mathrm{var}} \mathrm{PEL}^{\mathrm{B}}$, uirtus sit dei 89 , uirtutis dei sit $\mathrm{PEL}^{\mathrm{A}}$; + in uobis $5^{1}$ et non ex nobis ] et non ex hominibus 89

4:8 Present: 515458616467757677788889 271 AMst PEL SPM SPE in omnibus ] in omni uel omnibus 77, om. 89 ; + enim 5861 tribulationem $\mathrm{SPM}^{\mathrm{var}}$ ] tribulatio 7576 , pressuram $\mathrm{AMst} \mathrm{PEL}^{\mathrm{B}}$, adtribulationem SPM ${ }^{\text {ed; }}$ + uel pressuram 77

patimur $\mathrm{SPM}^{\mathrm{var}}$ ] om. 7576 , passi $89 \mathrm{AMst} \mathrm{SPM}^{\mathrm{ed}}$; + uel tribulati 77 sed non ] om. 89

angustiamur 64 ] coangustiamur 77 , coangustiati $A M s t^{e d} \mathrm{SPM}^{\mathrm{var}}$, angustiati $\mathrm{AMst}^{\mathrm{var}} \mathrm{SPM}^{\mathrm{ed}}$

aporiamur 67 ] om. 89, adperamur $271^{*}$, inopiam passi AMst, aperiamur $\mathrm{PEL}^{\mathrm{B}} \mathrm{SPE}^{\mathrm{var}}$, confusi SPM, operiamur SPE ${ }^{\mathrm{var}}$

sed non ] et non 89

destituimur $617888^{*} 271$ ] exaporiamur 64, exporiamur 89, destituti AMst, aporiamur $\mathrm{SPM}^{\mathrm{ed}}$, operiamur $\mathrm{SPM}^{\mathrm{var}}$, aperiamur $\mathrm{SPM}^{\mathrm{var}}$; + uel destituti 77

4:9 Present: 515458616467757677788889 271 AMst PEL SPM SPE

persecutionem patimur 88 ] persecuti uel persecutionem patimur 77 , persecutionem passi $\mathrm{AMst}^{\mathrm{ed}}$, persecutiones passi $\mathrm{AMst}^{\mathrm{A}}$, om. $\mathrm{PEL}^{\mathrm{A}}$, persecutionem patiamur $\mathrm{SPM}^{\mathrm{var}}$

sed non ] et non 89 , om. PEL ${ }^{\mathrm{A}}$

derelinquimur ] delinquimur 64 , derelicti uel non derelinquimur 77 , reliquimur $2{ }^{\mathrm{C}} \mathrm{C}$, derelicti $\mathrm{AMst}{ }^{\mathrm{ed}}$, angustiati $\mathrm{AMst}^{\mathrm{A}}$, om. $\mathrm{PEL}^{\mathrm{A}}$

\pm humiliamur sed non confundimur $5861 \mathrm{PEL}^{\mathrm{B}} \mathrm{SPE}^{\mathrm{var}}$

deicimur ] tribulamur $61 \mathrm{PEL}^{\mathrm{B}}$, deiecti uel deicimur 77 , deiecti AMst sed non ] et non 89

perimus ] + uel perditi 77

4:1O Present: 515458616467757677788889 271 AMst PEL SPM SPE semper ] sed per 54

mortificationem (88) ] mortem 75767789 AMst SPM

iesu ] christi 75767778 89, domini iesu $\mathrm{AMst}^{\mathrm{R}}$, om. PEL ${ }^{\mathrm{var}}$, domini nostri iesu christi SPM ${ }^{\text {ed }}$, domini iesu christi SPM ${ }^{\text {var }}$

in corpore nostro ] in cordibus nostris $271^{*}$, in corpore $\mathrm{AMst}^{\mathrm{R}} \mathrm{PEL}^{\mathrm{var}}$ circumferentes

ut et uita ] ut uitam SPM ${ }^{\mathrm{var}}$ 
iesu ] christi iesu 61 SPM $^{\text {ed }}$, iesu christi 75767789 PEL $^{\mathrm{B}} \mathrm{SPM}^{\mathrm{var}}$ in corporibus nostris manifestetur $\left.\left(54^{*}\right)\langle 67\rangle \mathrm{PEL}^{\mathrm{var}}\right]$ in corpore nostro manifestetur 617576777889 AMst PEL ${ }^{\mathrm{AB}} \mathrm{SPM}^{\mathrm{ed}}$, in corporibus manifestetur 271, manifestetur in carne nostra mortali SP $\mathbf{M}^{\mathrm{var}}$

4:11 Present: $5^{1} 545^{8^{\dagger}} 616467757677788889$ 271 AMst PEL SPM SPE semper $\mathrm{AMst}^{\mathrm{R}} \mathrm{SPM}^{\mathrm{var}}$ ] si $61777889 \mathrm{AMst}^{\mathrm{ed}}$, sic $\mathrm{SPM}^{\text {ed }}$ enim $\mathrm{AMst}^{\mathrm{R}}$ ] om. 64 271, ergo AMsted

nos qui uiuimus 271 ] nos uiuentes uel qui uiuimus 77 in mortem tradimur ] in morte tradimur $5^{2} 89271 \mathrm{SPM}^{\mathrm{var}} \mathrm{SPE}^{\mathrm{var}}$ propter iesum ] per iesum 89 ut et ] ut 61271 PEL $^{\text {var }}$, et AMst ${ }^{\text {var }}$, et ut AMst ${ }^{\text {var }}$ uita iesu (89) ] haec uita iesu 271 ; + christi 757677 manifestetur ] om. $58^{1}$, palam fiat AMst in carne nostra mortali ] in mortali carne nostra $6175767_{77} \mathrm{PEL}^{\mathrm{B}}$, in carne mortali nostra 64, in hac mortali carne nostra AMsted, in hoc mortale carne nostra AMstrar, in corpore nostro SPM ${ }^{\mathrm{var}}$, in carne nostra mortalia $\mathrm{SPE}^{\mathrm{var}}$ + + manifestetur $5^{8^{1}}$

4:12 Present: 515458616467757677788889 271 AMst PEL SPE ergo ] i $[t a]$ ut 64, itaque 75767789 AMst mors ] + quidem $\mathrm{AMst}^{\mathrm{R}}$ in nobis ] in ụobis 58 operatur ] operetur 64 uita ] lac. 67, uitam 271 autem $\langle 67\rangle$ ] uero $\mathrm{AMst}^{\mathrm{R}}$ in uobis ] in nobis $5^{8} 78$

4:13 Present: 515458616467757677788889 271 AMst PEL SPE habentes 271 ] habemus AMst ${ }^{\mathrm{R}}$ autem $\mathrm{AMst}^{\mathrm{R}}$ ] uero AMsted, igitur PEL ${ }^{\text {var }}$, om. $\mathrm{PEL}^{\text {var }}$ eundem ] eadem 75 , secundum AMst ${ }^{\text {var }}$ spiritum fidei ] spiritum in fide 89 sicut ] secundum 6477 AMst, ut PEL ${ }^{\mathrm{B}}$; + quod 6477 AMst scriptum est $64\langle 67\rangle 271$ ] dictum est $\mathrm{PEL}^{\mathrm{B}}$; + in psalmo cxv 61 credidi $\left(88^{*}\right)$ ] lac. 67 propter quod ] propter 88 ; + et 77

$\dagger{ }_{2}$ Cor. 4:11. This verse is duplicated in $\mathrm{VL}_{5} 8$, with two differences. 
locutus sum $\langle 67\rangle$

et nos credimus ] lac. 67 , et nos credidimus 75 PEL ${ }^{\mathrm{A}}$

propter ] ideo $61 \mathrm{PEL}^{\mathrm{B}}$, lac. 67 , ideo uel propter 77

quod ] om. 61, lac. 67, quae $P E L^{\text {var }}$

et loquimur ] lac. 67, loquimur AMsted $\mathrm{PEL}^{\mathrm{var}}$

4:14 Present: 5154586164757677788889 271 AMst PEL

scientes quoniam $\mathrm{AMst}^{\mathrm{var}}$ ] scientes quia $89 \mathrm{AMst}^{\mathrm{var}}$, scientes quod AMsted PEL var

qui suscitauit ] qui suscitabit 64271 , suscitauit 78 ; + a mortuis AMstvar iesum ] dominum iesum $547576777889 \mathrm{AMst}^{\mathrm{PEL}}{ }^{\mathrm{B}}$, dominum nostrum iesum christum 61

et nos ] et non $54^{*}$

cum iesu (75) ] per iesum AMst ${ }^{\mathrm{R}}$, cum illo PEL var

suscitabit AMst ${ }^{\mathrm{var}}$ ] suscitauit 757689 AMstrar $^{\text {vEL }}{ }^{\text {var }}$, exsuscitabit AMst ${ }^{\text {ed }}$ et constituet ] et constituit $5489271^{*} \mathrm{AMst}^{\mathrm{var}} \mathrm{PEL}^{\mathrm{var}}$, et stare faciet 64 , et constituit uel constituet 77 ; + nos 51

uobiscum $\left\langle 5^{8}\right\rangle$

4:15 Present: $5^{1} 54586164757677788889$ 271 AMst PEL

omnia enim propter ] omnia autem propter $5_{1} \mathrm{PEL}^{\text {var }}$

uos ] om. 78 ; + patimur $6177^{\mathrm{C}}$

ut gratia ] ut gratiam 271, ut donum $\mathrm{AMst}^{\mathrm{R}}$, om. $\mathrm{AMst}^{\mathrm{var}}$

abundans per $5154^{*} 586188$

multos ] plurimam $6177 \mathrm{PEL}^{\mathrm{B}}$, multarum $64 \mathrm{AMst}^{\mathrm{var}}$, multorum AMsted, multas PEL ${ }^{\text {var }}$; in $5^{8}$

gratiarum ] om. $\mathrm{PEL}^{\mathrm{B}}$

actione 54 ] actionem 6164757677 AMst PEL $^{\mathrm{B}}$, actio 78 271, actiones PEL var abundet $51\left(54^{*}\right) 58617888$ ] alium det 271

in gloriam dei ] in gloria dei $6189271 \mathrm{PEL}^{\mathrm{B}}$, ad gloriam dei $\mathrm{AMst}^{\mathrm{R}}$

4:16 Present: 515458616467757677788889 271 AMst PEL CAr SPE

propter quod non ] lac. 67, propterea non 7576 89, quapropter non AMst, propter quia non PEL ${ }^{\text {var }}$, abs. CAr

deficimus AMst ${ }^{\text {var }}$ ] deficiamus 6189 AMst $^{\text {ed }}$, infirmemur 64, lac. 67, deficimur 271, defecimus AMstvar, abs. CAr

sed licet ] licet enim 61 PEL $^{\mathrm{B}}$ CAr, sed [si] 64, lac. 67, sed et si 7576 89, sed si 77 , licet si AMsted, et licet $\mathrm{AMst}^{\mathrm{R}}$, licet $\mathrm{PEL}^{\mathrm{var}}$

is 5488271 ] om. $616475767789 \mathrm{AMst} \mathrm{PEL}^{\mathrm{B}}$, lac. 67

qui foris est $\mathrm{AMst}^{\mathrm{R}}$ ] exterior $61\langle 64\rangle 77 \mathrm{AMst}^{\mathrm{ed}} \mathrm{PEL}^{\mathrm{B}}$, lac. 67, qui fortis est 89 
noster homo ] homo noster 5461647576 AMst SPE, lac. 67

corrumpitur 61 AMsted ${ }^{e d}$ corrumpatur $5^{1} 5^{8}$ (271) AMst ${ }^{\mathrm{R}} \mathrm{SPE}$, lac. 67, interit 89 ; + confunditur se CArvar

tamen is qui intus est 5488 ] sed interior $6164 \mathrm{AMst}^{\text {ed }} \mathrm{PEL}^{\mathrm{B}} \mathrm{CAr}^{\mathrm{ed}},[\ldots]$ intus

[...] 67, sed qui intus est $757689 \mathrm{PEL}^{\text {var }}$, sed interior noster 77 , is qui intus est 271, interior tamen $\mathrm{AMst}^{\mathrm{R}}$, sed tamen interior PEL ${ }^{\mathrm{var}}$, interior CArvar renouatur 64 ] [4] atur $54^{*}$, lac. 67

de die in diem ] lac. 67 , die ac die 77 , de die in die $271 \mathrm{CAr}$

4:17 Present: 515458616467757677788889 271 AMst PEL SPE id enim quod $\left.\left(54^{*}\right)\langle 67\rangle \mathrm{PEL}^{\mathrm{var}}\right]$ nam quod $6175\langle 76\rangle 7789 \mathrm{PEL}^{\mathrm{B}}$, quod enim 64 in ] ad 6477, om. $88271 \mathrm{PEL}^{\mathrm{B}}$ praesenti $5154\langle 58\rangle 6167767888271$ ] praesens $6477{ }^{P E L^{B}}$ est ] om. $64271 \mathrm{AMst}^{\mathrm{var}} \mathrm{PEL}^{\mathrm{var}}$, lac. 67

momentaneum $\langle 67\rangle$ ] temporale $64 \mathrm{PEL}^{\mathrm{B}}$, ad tempus 77, om. $271 ;+$ est 6477 PEL ${ }^{\text {var }}$

et leue ] leue 64, om. 271; + est AMst ${ }^{\text {ed }}$

tribulationis $\langle 67\rangle$ ] pressurae uel tribulationis 77 , om. 271, pressurae AMst $P E L^{B}$, tribulationes $\mathrm{SPE}^{\mathrm{var}}$ nostrae $51545^{8} 77$ ] lac. 67, om. 271 PEL Par $^{\text {va }}$ supra modum ] iuxta incredibilem modum et 64 , secundum excellentiam 7576 , om. 271

in sublimitatem $\langle 67\rangle$ ] in sublimitate ${ }_{54}{ }^{\mathrm{C}} 757^{6} 89 \mathrm{AMst}^{\mathrm{var}} \mathrm{PEL}^{\mathrm{B}} \mathrm{SPE}^{\mathrm{var}}$, om. 61 271, in incredibilem modum 64, sublimitatem PEL ${ }^{\text {var }}+$ gloriae PEL var aeternum 51587888 ] aeternam $54\langle 67\rangle \mathrm{PEL}^{\mathrm{var}}$, aeternae 757689 , om. 271 gloriae pondus $515458(89) \mathrm{AMst}^{\mathrm{R}}$ ] pondus gloriae $6177 \mathrm{AMst}^{\mathrm{ed}} \mathrm{PEL}^{\mathrm{var}}$, om. 271, pondus PEL var

operatur nobis AMstvar $]$ operatur in uobis 51 , operatur in nobis 54586167 757677788889271 AMst $^{\text {ed }}$ PEL SPE

4:18 Present: $51545^{8} 616467757677788889$ 271 AMst PEL SPE

non contemplantibus AMst ${ }^{\mathrm{var}}\left(\mathrm{PEL}^{\mathrm{A*}}\right)^{\mathrm{C}}$ ] non respicientibus 64 , non respicientes $757689 \mathrm{PEL}^{\mathrm{var}}$, non contemplantes $77 \mathrm{AMst}^{\mathrm{ed}} \mathrm{PEL}^{\mathrm{B}}$, non contemplatur 271

nobis $\mathrm{AMst}^{\mathrm{D}}$ ] om. $6475^{*} 767789 \mathrm{AMst}^{\mathrm{ed}} \mathrm{PEL}^{\mathrm{B}}$, lac. 67 , nostrorum $75^{\mathrm{C}}$ quae uidentur $51545^{8} 61\langle 67\rangle 88$ ] quae uident $75^{*}$, om. $77 \mathrm{SPE}^{\mathrm{var}}$, quae uidetur 271

sed quae non 5154586188271 ] quae $77 \mathrm{SPE}^{\mathrm{var}}$, sed quia non $\mathrm{PEL}^{\mathrm{B}}$ uidentur $\langle 67\rangle$ 
quae enim uidentur $51545861\langle 67\rangle 271$ ] temporalia 77 , quae uidentur enim PELvar

temporalia sunt $\langle 67\rangle]$ temporalia 7576 , ad tempus sunt 7789

quae autem $51545861\langle 67\rangle 7788$ ] quae $271 \mathrm{PEL}^{\text {var, }}$, quae uero AMst, nam quae $P E L^{B}$

non uidentur AMst $\left.{ }^{\text {var }}\right]$ non cernuntur AMsted

aeterna sunt $51545^{8} 7778(88) 271$ ] aeterna 6477 AMst $^{\text {var }}$, aeter[... 67

5:1 Present: $5^{1} 545^{86164677576777888} 89$ 271 AMst PEL SPM

scimus enim ] scimus autem 88 AMst PEL ${ }^{\mathrm{A}}$, scimus PEL ${ }^{\text {var }}$

quoniam si $\mathrm{AMst}^{\mathrm{var}}$ ] quia si $64 \mathrm{AMst}^{\mathrm{ed}}$, si PEL var

terrestris ] terrena $64 \mathrm{SPM}^{\mathrm{var}}$

domus nostra $\left.\left\langle 5^{1}\right\rangle\right]$ nostra domus 6477 , domus nostrae $\mathrm{AMst}^{\mathrm{D}}$, domum PELvar

huius habitationis $\left(271^{*}\right)$ ] habitationis 64

dissoluatur ] om. $61^{*}$, [de]soluitur $61^{\mathrm{C}}$, resoluatur $64 \mathrm{SPM}^{\text {var }}$, desoluatur 77 , dissoluantur 271

quod $\mathrm{AMst}^{\mathrm{AC}} \mathrm{AMst}^{\mathrm{R}}$ ] om. 64, quia $\mathrm{AMst}^{\mathrm{ed}}$

aedificationem $51545^{8} 88271$ ] habitaculum 6177 , ad aedificationem 67 ; + habemus 64

ex deo ] a deo SPM

habeamus PEL ${ }^{\text {var }}$ SPM $^{\text {var }}$ ] habemus $51586175767778271 \mathrm{PEL}^{\mathrm{AB}}$ SPM $^{\mathrm{ed}}$, om. $64 \mathrm{PEL}^{\mathrm{var}}$

domum non ] donum non $75^{*}$

manufactam SPM ${ }^{\mathrm{var}}$ ] manufacta 64271 , factam SPM${ }^{\mathrm{ed}}$; + sed 586189271 PEL var

aeternam in caelis 515458677788 ] aeterna in caelis amen 271 , in caelis PELvar

5:2 Present: 515458616467757677788889 AMst PEL SPM

nam et ] etenim 616477 AMst PEL $^{\mathrm{B}}$, nam PEL ${ }^{\text {var }}$ SPM, om. PEL var

in hoc ] hoc 6789 AMst $^{\text {var }}$

ingemescimus $515458\langle 64\rangle 78$ ] gemimus 757689 SPM

habitationem nostram quae 5154586177 ] habitaculum nostrum quod $\langle 64\rangle$

757689 SPM, habitatione nostra quae 88 , habitaculo nostro quod AMst ${ }^{\text {ed }}$, habitaculo quod AMst ${ }^{\text {var }}$, habitationem quae PEL var

de caelo est 54587778 ] in caelo est 51

superindui $\mathrm{SPM}^{\mathrm{var}}$ ] superinduere $61 \mathrm{PEL}^{\mathrm{var}}$, superinduere uel superindui

(77), semper indui AMst ${ }^{\mathrm{var}}$, superinducere $\mathrm{PEL}^{\mathrm{B}}$, superuestiri SPM ${ }^{\text {ed }}$

cupientes 
5:3 Present: 515458616467757677788889 AMst PEL SPM

si tamen ] si [...] 64, siquidem AMst; + et 647576

uestiti $78 \mathrm{PEL}^{\text {var }}$ ] expoliati $61777^{\mathrm{gl}} \mathrm{PEL}^{\mathrm{AB}}$, induti 6489 AMst, spoliati 7576 SPM, expoliati corpore PEL ${ }^{\text {var }}$ + et 5188 SPM $^{\text {var }}$

non nudi inueniamur ] nudi non inueniamur PEL var

5:4 Present: 5154586164677576777888 89 AMst PEL sPM

nam et ] etenim $61\langle 64\rangle 77 \mathrm{PEL}^{\mathrm{B}}$, nam AMst $\mathrm{SPM}^{\mathrm{var}}$, om. PEL ${ }^{\mathrm{var}}$

qui ] cum $6177 \mathrm{AMst}^{\mathrm{ed}} \mathrm{PEL}^{\mathrm{B}}$, dum $\mathrm{AMst}{ }^{\mathrm{AD}}$

sumus in ] sum in AMst ${ }^{\text {var }}$, in PEL var

tabernaculo PEL var ] hoc habitaculo 5189 , tabernaculo isto 58 , corpore isto $61 \mathrm{PEL}^{\mathrm{B}}$, hanc habitationem $\left(64^{*}\right)^{\mathrm{C}}$, hoc corpore $67 \mathrm{AMst}^{\mathrm{R}} \mathrm{PEL}^{\mathrm{A}}$, habitaculo isto 7576 , habitaculo hoc $77 \mathrm{SPM}$, tabernaculo hoc 78 , hoc tabernaculo isto $88^{*}$, hoc tabernaculo $88^{\mathrm{C}} \mathrm{PEL}^{\mathrm{var}}$, isto corpore AMst ${ }^{\mathrm{ed}}$, hoc sumus corpore PEL var

ingemescimus $5154\langle 64\rangle 7678$ ] gemimus $89 \mathrm{sPM}$

grauati 64 ] onerati 757689

eo quod ] in quo 647576 , in eo quod SPM

nolumus ] nolimus 61, uolumus $\mathrm{AMst}^{\mathrm{A}}$

expoliari $\langle 64\rangle 75>678$ ] spoliari $6177 \mathrm{PEL}^{\mathrm{var}} \mathrm{SPM}$, [3]poliari 88*, exui AMst, grauari PELA

sed superuestiri ] sed superindui AMst, sed suprauestire PEL ${ }^{\text {var }}$, sed superuestire PELvar; + cupientes SPM

ut absorbeatur ] ut absorbeatis $54^{*}$

quod mortale est ] mortale hoc 617789 AMst PEL $^{B}$ SPM, mortale $\langle 64\rangle 7576$, hoc mortale PEL var

a uita ] + expoliari sed superuestiri ut absorbeatur mortale hoc a uita $89 *$

5:5 Present: 5154586164677576777888 89 AMst PEL sPM

qui autem ] quis autem $\mathrm{AMst}^{\mathrm{A}}$

efficit $5^{8}$ ] perficit 6177 AMst $P E L^{B}$, operatus est 64, effecit PEL var

nos ] lac. 64

in hoc ipsum ] in hoc ipsud 89

deus ] + est $\mathrm{AMst}^{\mathrm{R}}$

qui ] + et $\mathrm{AMst}^{\text {ed }} \mathrm{PEL}^{\text {var }}$, + etiam $\mathrm{AMst}^{\mathrm{R}}$

dedit nobis pignus $\langle 64\rangle \mathrm{SPM}^{\mathrm{var}}$ ] dedit nobis arram $\mathrm{SPM}^{\mathrm{ed}}$, dedit nobis arma $\mathrm{SPM}^{\mathrm{var}}$

spiritus ] spiritum 64 AMst 
5:6 Present: 5154586164677576777888 89 AMst PEL

audentes ] audientes $5458768889^{\mathrm{C}}$, gaudentes 61 , confidentes 64 , audeientes $89^{*}$, fidentes AMst

igitur ] ergo 64

semper et scientes $\langle 64\rangle(75)$

quoniam ] quod 757689 , quia AMst $\mathrm{PEL}^{\mathrm{A}}$

dum sumus ] quamdiu sumus 58 , cum sumus 6177 , inmanentes 64 , inhabitantes AMst ${ }^{\text {ed }}$, habitantes AMst ${ }^{\text {var }}$; + uel peregrinamur 77

in corpore ] in corpora isto 61, corpore 64, in hoc corpore $88 \mathrm{AMst}^{\mathrm{R}} \mathrm{PEL}^{\mathrm{var}}$ peregrinamur $61\langle 64\rangle$

a domino ] a deo 61757677

5:7 Present: 515458616467757677788889 AMst PEL

per fidem enim ambulamus $\langle 64\rangle$

et ] om. 647576 AMst

non per speciem ] non per spem $5^{1} 5^{8}$

5:8 Present: 5154586164677576777888 89 AMst PEL

audemus ] audiemus 61 , confidi [mus] 64 , audemur $77^{\mathrm{C}}$

autem ] ergo 61 $\mathrm{AMst}^{\mathrm{ed}}$, etiam 75 76, ergo uel autem 77, om. $\mathrm{AMst}^{\mathrm{R}}$

et ] om. 7576

bonam uoluntatem habemus $54^{*}\langle 67\rangle 78$ ] consentimus $61777^{8{ }^{\mathrm{gl}}}$ AMst $\mathrm{PEL}^{\mathrm{B}}$, bene arbitramur 64 , uoluntatem habentes 7576 , tam uoluntatem habemus $89^{*}$, tam uoluntatem habentes $89^{\mathrm{C}}$, bonam uoluntatem habeamus PEL var

magis peregrinari $67\langle 64\rangle]$ magis peregrinare $75 \mathrm{PEL}^{\mathrm{var}}$, magis peregrinamur AMst $^{\text {var }}$ PEL $^{\text {var }}$

a corpore et ] de corpore et $6177 \mathrm{AMst}^{\text {var }} \mathrm{PEL}^{\mathrm{B}}$

praesentes esse $51545^{8} 6167767889$ ] [in]maner[e] 64, adesse $777^{8 \mathrm{gl}} \mathrm{PEL}^{\mathrm{B}}$ ad deum ] ad dominum $5^{1}\langle 64\rangle 7576777889{\text { AMstrar }{ }^{\text {PEL }}}^{\mathrm{A}}$

5:9 Present: 5154586164677576777888 89 AMst PEL

et ideo $\mathrm{AMst}^{\mathrm{R}}$ ] et 7576 89, ideo et 77 , ideo AMst ${ }^{\text {ed }}$, ideo enim AMst ${ }^{\text {var }}$

contendimus AMst ${ }^{\mathrm{var}}$ ] abimus 64, conuicimur 77, enitamur AMsted, imitamur $\mathrm{AMst}^{\mathrm{A}}$, conamur AMstvar, conitimur $\mathrm{PEL}^{\mathrm{B}}$, contendamus PEL ${ }^{\text {var }}$ siue absentes ] lac. 64 , siue praesentes $7576 \mathrm{AMst}_{\mathrm{PEL}}{ }^{\mathrm{var}}$, siue peregrinantes 89

siue praesentes 5154586167777889 ] siue peregrinantes $\langle 64\rangle$ AMst, siue absentes 7576 PEL var

placere illi ] lac. 64, placentes illi 77 ; + [esse] 64, + sitis 77 
5:10 Present: 515458616467757677788889 AMst PEL tes omnes enim $\langle 64\rangle]$ nam omnes 757689 , omnes TES ${ }^{\text {ed }}$, om. TES var $^{\text {var }}$ nos manifestari oportet $\langle 64\rangle]$ nos manifestare oportet ${ }_{51} \mathrm{PEL}^{\mathrm{var}}$, manifestari oportet $54 \mathrm{TES}^{\mathrm{var}}$, nos oportet manifestari 61, uos manifestari oportet 75 76, nos oportet AMst ${ }^{\mathrm{R}}$, manifestari nos oportet $\mathrm{AMst}^{\mathrm{var}}$, oportet nos stare TES ${ }^{\text {var }}$, uos manifestare oporteat TES $\mathrm{S}^{\mathrm{var}}$

ante tribunal christi $(75)$ ] ante tribunal domini TES ${ }^{\text {var }}$; + assistere $\mathrm{AMst}^{\mathrm{R}}$ ut referat $\left.\mathrm{PEL}^{\text {var }}\right]$ [2]f[erat] 64, ut ferat 7576 89, ut recipiat uel reportet 77 , ut recipiat AMst PEL ${ }^{\mathrm{A}}$ TES $^{\mathrm{var}}$, ut reportet $\mathrm{PEL}^{\mathrm{B}}$ TES $^{\text {ed }}$ unusquisque $\langle 64\rangle]+$ uestrum $\mathrm{TES}^{\mathrm{var}}$, + nostrum $\mathrm{TES}^{\mathrm{var}}$ propria corporis 78 ] propria corporis sui $61, \mathrm{om} .64$, quae per corpus $7^{8 \mathrm{gl}}$, facta corporis $\mathrm{AMst}^{\mathrm{R}}$, sui corporis propria TES $\mathrm{S}^{\mathrm{ed}}$, sui corporis TES $\mathrm{S}^{\mathrm{var}}$ prout gessit $]$ secund $[u] \mathrm{m}[e a]$ quae per $\mathrm{c}[o]$ rpus ge $[s s i t] 64$, secundum quod gessit 757689 , ut gessit AMstrar, tunc quod gessit TES ${ }^{\text {var }}$, secundum quae egit TES ${ }^{\text {ed }}$

siue bonum siue malum $\langle 64\rangle 67$ ] siue bona siue mala AMst ${ }^{\text {ed }}$ TES $^{\text {ed }}$, seu bona seu mala $\mathrm{AMst}^{\mathrm{A}}$, bona siue mala $\mathrm{TES}^{\mathrm{var}}$

5:11 Present: $3^{2} 5^{1} 545^{8} 6164677576777888$ 89 AMst PEL CAr scientes ergo ] fratres scientes 32 , scientes igitur $\langle 64\rangle 757689$ AMst timorem domini ] timorem dei 89 hominibus suademus deo autem $\langle 64\rangle]$ hominibus suademur deo autem CArvar

manifesti sumus $\left.\left\langle 3^{2}\right\rangle \mathrm{AMst}^{\mathrm{var}}\right]$ manifestati sumus $5861\langle 64\rangle \mathrm{AMst}^{\mathrm{ed}}$ spero autem AMstrar ${ }^{\mathrm{va}}$ spero $\mathrm{AMst}^{\mathrm{ed}}$, abs. CAr

et in ] lac. 64, in 89, abs. CAr

conscientiis uestris $\langle 32\rangle 5488$ ] lac. 64 , conscientiis nostris AMst ${ }^{\mathrm{var}}$, abs. CAr manifestos $\left.\left\langle 3^{2}\right\rangle\langle 64\rangle\right]$ nos manifestos $77 \mathrm{AMst}^{\text {ed }} \mathrm{PEL}^{\mathrm{B}}$ abs. CAr nos AMstrar ] lac. 32 64, non 67, om. $77 \mathrm{AMst}^{\mathrm{ed}} \mathrm{PEL}^{\mathrm{B}}$, uos 89, abs. CAr esse $\langle 32\rangle]$ lac. $64, a b s$. CAr

5:12 Present: $3^{2} 5^{1} 545^{8} 6164677576777888$ 89 AMst PEL

non iterum $\left.\left\langle 3^{2}\right\rangle\right]$ lac. 64 , non igitur $\mathrm{PEL}^{\mathrm{B}}$

nos commendamus 3254 ] [nos]met ipsos comme[ndamus] 64, commendamus nosmet ipsos 78, nos commodamus AMst ${ }^{\text {var }}$, commendamus nos PEL ${ }^{\text {var }}$

uobis sed occasionem $\left.757789^{C *}\right]$ uobis $51,[\ldots]$ casionem 64

damus uobis $]$ om. 51 , uobis damus $61, \mathrm{~d}[a] \mathrm{n}[$ tes ... $]$ 64, dantes nobis 7576 , dantes uobis 77 , damus $\mathrm{PEL}^{\mathrm{B}}$

gloriandi ] lac. 64, gloriam AMst ${ }^{\mathrm{var}}$ 
pro nobis ] lac. 64, pro uobis $77 \mathrm{PEL}^{\mathrm{B}}$, de nobis $\mathrm{AMst}^{\mathrm{R}}$ ut ] lac. 64, sed 77; + nos 61, + aliquid AMst ${ }^{\mathrm{R}}$

habeatis ad 3258 ] lac. 64 , habeamus aduersus $\mathrm{AMst}^{\mathrm{R}}$ eos qui in facie ] lac. 64, eos qui in faciem 89 gloriantur $\langle 32\rangle$ ] lac. 64, glorificantur uel glorificantes 77 et non in corde $\langle 32\rangle]$ lac. 64

5:13 Present: 3251545861677576777888 89 AMst PEL siue enim AMstrar $]$ siue ergo AMst ${ }^{\text {ed }}$ mente excedimus AMst ${ }^{\mathrm{var}}$ ] mente excidimus $32546777^{*} \mathrm{AMst}^{\mathrm{ed}} \mathrm{PEL}^{\mathrm{A}}$, excidimus mente 7589 , excedimus mente 76 deo siue ] siue $\mathrm{AMst}^{\mathrm{var}}$ sobrii sumus ] sumus sobrii 51 , sanum sapimus AMst ${ }^{\text {ed }}$, sanum sapiamus $\mathrm{AMst}^{\mathrm{var}}$ uobis ] om. PEL $^{\text {var }}$; sapimus AMst ${ }^{\mathrm{AD}}$

5:14 Present: $3^{2} 5^{1} 545^{8} 616467757^{6} 777888$ 89 AMst PEL caritas enim christi ] lac. 64 urget nos $3254^{c} 677576777888$ ] arguet nos $54^{*}$, lac. 64, urget uos $\mathrm{PEL}^{\mathrm{B}}$ aestimantes hoc 51586788 ] iudicantes hoc $3261757677 \mathrm{PEL}^{\mathrm{B}}$, aestimantes autem hoc $\left(54^{*}\right)^{\mathrm{C}}$, lac. 64 , hoc iudicantes AMsted, certe hoc iudicantes $\mathrm{AMst}^{\mathrm{D}}$; + iudicantes PEL ${ }^{\mathrm{var}}$

quoniam ] quod $6177 \mathrm{AMst}^{\mathrm{PEL}} \mathrm{B}^{\mathrm{B}}$, lac. 64 , quia 78 si $\mathrm{AMst}^{\mathrm{var}} \mathrm{PEL}^{\mathrm{var}}$ ] om. $6175767789 \mathrm{AMst}^{\mathrm{ed}} \mathrm{PEL}^{\mathrm{AB}}$, lac. 64 unus pro omnibus mortuus $\langle 32\rangle\langle 64\rangle$ est ergo omnes mortui $\langle 64\rangle]$ ergo omnes mortui PEL ${ }^{\text {var }}$ sunt ] lac. 64 , sumus 89

5:15 Present: $3251545^{8} 6164677576777888$ 89 AMst PEL FOR et ] om. $\mathrm{FOR}^{\mathrm{ed}}$, christus $\mathrm{FOR}^{\mathrm{var}}$

pro omnibus ] pro omnes 89 ; + uero $\mathrm{AMst}^{\mathrm{R}}$, + et $\mathrm{FOR}^{\mathrm{var}}$ mortuus est $\langle 64\rangle]$ + christus $5458647778 \mathrm{AMst}^{\mathrm{var}}$, + unus 61, + ergo omnes mortui sunt et pro omnibus mortuus est 88 ut et (54) FOR ${ }^{\text {var }}$ ] ut $516475767788^{*} 89 \mathrm{PEL}^{\mathrm{B}} \mathrm{FOR}^{\text {ed }}$, et AMst ${ }^{\mathrm{var}}$, om. FOR ${ }^{\mathrm{var}}$ qui uiuunt (64) ] uiuentes 77 iam non $\mathrm{AMst}^{\mathrm{var}}$ ] non 7576 89, non iam $77 \mathrm{AMst}^{\text {ed }}$, iam PEL ${ }^{\text {var }}$ sibi uiuant ] sibi ipsi uiuant 7576 89, sibi uiuunt 78 PEL $^{\text {var }}$ sed ] lac. 64 ei qui pro ] pro ei $77^{*}$, pro $77^{\mathrm{C}}$, illi qui pro $89 \mathrm{FOR}^{\mathrm{var}}$ ipsis ] illis $7576 \mathrm{FOR}^{\mathrm{var}}$, eis $77 \mathrm{PEL}^{\mathrm{var}}$, ipsi $\mathrm{PEL}^{\mathrm{B}}$; + et FOR ${ }^{\mathrm{var}}$ 
mortuus est ] mortuo 77

et resurrexit $54\langle 64\rangle$ ] et surrexit 7576 , et resurgenti 77 , et reuixit $\mathrm{FOR}^{\mathrm{var}}$

5:16 Present: $3^{2} 5^{1} 545^{8} 6164677576777^{8} 88$ 89 AMst PEL

itaque ] igitur 757689

nos ] om. 51 AMst $^{\mathrm{R}}$

ex hoc neminem nouimus ] neminem nouimus $32 \mathrm{PEL}^{\mathrm{A}}$, amodo neminem nouimus $\langle 64\rangle$ (75) 76 89, ex hoc uel a nunc neminem nouimus 77, ex hoc tempore neminem nouimus AMsted, neminem ex hoc nouimus $\mathrm{AMst}^{\mathrm{R}}$, neminem nouimus ex hoc $\mathrm{PEL}^{\mathrm{B}}$, ex hoc nos neminem PEL ${ }^{\text {var }}$

secundum carnem

et si ] quamuis 89 , si PEL var

cognouimus ] noue[ra]mus 64

secundum carnem christum ] christum secundum carnem 517576

sed nunc iam non $\langle 64\rangle$ ] sed nunc non iam 77, sed nunc non AMst ${ }^{\text {var }}$

nouimus AMst ${ }^{\text {var }}$ ] cognoscimus AMst ${ }^{\text {ed }}$

\pm eum secundum carnem esse 61, secundum carnem 757677

5:17 Present: $3^{2} 5^{1} 545^{8} 6164677576777888$ 89 AMst PEL

si qua ergo ] si qua $61^{*}$, igitur si qua 64, itaque si qua 75767789 , quare si qua est AMst

in christo noua creatura $(51)\langle 64\rangle]+$ est $54^{\mathrm{C}},+$ intellegens PEL ${ }^{\text {var }}$

uetera transierunt $\langle 32\rangle]+$ et AMst ${ }^{\text {ed }}$

ecce facta sunt $\left.\left\langle 3^{2}\right\rangle\langle 64\rangle\right]$ facta sunt $\mathrm{AMst}^{\text {var }}$

noua $\left.\left\langle 3^{2}\right\rangle\right]$ omnia noua $54586189 \mathrm{AMst}^{\mathrm{ed}} \mathrm{PEL}^{\mathrm{B}}$

5:18 Present: $3^{2} 5^{1} 545^{8} 6164677576777^{8} 88$ 89 AMst PEL

omnia autem ex deo $\langle 64\rangle]$ om. 61, omnia ex deo AMst ${ }^{\text {var }}$ + gratias autem deo $61 \mathrm{PEL}^{\mathrm{B}}$

qui reconciliauit ] qui reconciliabit $64 ;$ + uel reconciliante uel reconciliare

77

nos sibi

per christum ] lac. 32, per iesum christum $\mathrm{AMst}^{\mathrm{R}} \mathrm{PEL}^{\mathrm{B}}$

et dedit $\langle 32\rangle\langle 64\rangle]$ deditque $\mathrm{AMst}^{\mathrm{R}}$; + uel dante 77

nobis ministerium reconciliationis $\langle 64\rangle$

5:19 Present: $3^{2} 5^{1} 545^{8} 6164677576777^{8} 88$ 89 AMst PEL quoniam quidem ] quidem 51 , quemadmodum quia 64 deus erat in christo $\langle 32\rangle]$ deus erat in christum PEL var mundum $\langle 64\rangle]$ saeculum 7576 
reconcilians sibi non $\langle 32\rangle 54$

reputans $\langle 64\rangle]$ imputans $\mathrm{AMst}^{\mathrm{R}}$

illis ] illi AMst ${ }^{\mathrm{var}} \mathrm{PEL}^{\mathrm{var}}$, om. PEL $^{\mathrm{var}}$

delicta ipsorum $\langle 32\rangle 61$ ] delicta eorum $5^{1} 647576$ 89, delicta illorum PEL ${ }^{\text {var }}$

et posuit in $\mathrm{AMst}^{\mathrm{R}}$ ] et ponens in $\langle 64\rangle 77 \mathrm{AMst}^{\mathrm{ed}} \mathrm{PEL}^{\mathrm{B}}$

nobis ] [2] bis 64, uobis $\mathrm{PEL}^{\mathrm{B}}$

\pm euangelium $617^{8 \mathrm{gl}}$, adnuntiationem $757^{6} 89$, euangelii 77

uerbum $\langle 32\rangle]$ uerba AMstvar

reconciliationis $\langle 32\rangle 54$ ] reconciliantis $\mathrm{PEL}^{\mathrm{B}}$; + doctrinae euangelii 58

5:20 Present: $3^{2} 51545^{8} 6164677576777888$ 89 AMst PEL

\pm et 58 , quod 77

pro christo ] pro quo christo 7576 , per christum $\mathrm{AMst}^{\mathrm{AD}}$

ergo $\langle 64\rangle \mathrm{AMst}^{\mathrm{R}} \mathrm{PEL}^{\text {var }}$ ] pro quo $61 \mathrm{AMst}^{\mathrm{ed}} \mathrm{PEL}^{\mathrm{AB}}$, om. 75767789 PEL ${ }^{\text {var }}$

legationem $\left\langle 3^{2}\right\rangle \mathrm{AMst}^{\mathrm{var}} \mathrm{PEL}^{\mathrm{var}}$ ] legatione $5^{1} 545^{8} 6175767888 \mathrm{AMst}^{\mathrm{ed}}$ $\mathrm{PEL}^{\mathrm{A}}$, leuatione PEL ${ }^{\mathrm{B}}$

fungimur 61$][f] \operatorname{ung}[i] \mathrm{m}[2] 3^{2}$, fungimus AMst ${ }^{\text {var }}$

tamquam 77

deo ] lac. 64, a deo 77 ; + uos AMst ${ }^{\mathrm{R}}$

exhortante per nos $5461\langle 64\rangle 7888$

obsecramus PEL var ] abs. 32, oramus 61, obsecrantes 757689 PELA $^{\mathrm{A}}$, orantes uel obsecrantes 77 , orantes $\mathrm{AMst}^{\mathrm{ed}} \mathrm{PEL}^{\mathrm{B}}$, rogamus $\mathrm{AMst}^{\mathrm{R}}$ pro christo $\langle 64\rangle]$ abs. 32 , per christum $6177 \mathrm{PEL}^{\text {var }}$; + uos AMst ${ }^{\text {var }}$ reconciliamini deo $\mathrm{PEL}^{\mathrm{var}}$ ] abs. 32, reconciliari deo 616475767789 AMst PEL ${ }^{A B}$

5:21 Present: 515458616467757677788889 AMst PEL

eum qui non ] om. 77 , eum enim qui $\mathrm{AMst}^{\mathrm{R}}$, eum qui cum non $\mathrm{PEL}^{\mathrm{B}}$

nouerat peccatum $\langle 64\rangle]$ cognoscentem peccatum 77 , cognouerit peccatum PEL $^{\mathrm{B}}$

pro nobis peccatum ] peccatum pro nobis $\mathrm{AMst}^{\mathrm{D}} \mathrm{PEL}^{\mathrm{var}}$

fecit $\left.78^{\mathrm{gl}}\right] \mathrm{f}[\ldots] 64$, factus est $78 \mathrm{AMst}^{\mathrm{var}}$, factum est PEL ${ }^{\mathrm{var}}$ ut nos ] ut PEL var

efficeremur ] essemus 6177 AMst PEL ${ }^{\text {var }}$, simus 64, efficemur 88*, efficeremus PEL var

iustitia dei 515488

in ipso ] lac. 67, illo 7576 89, ipso 88, per ipsum $\mathrm{AMst}^{\mathrm{R}}$, in ipsum $\mathrm{PEL}^{\mathrm{B}}$ 
6:1 Present: $3^{2} 5^{1} 545^{8} 6164677576777888$ 89 AMst PEL SPE \pm quin et $\mathrm{AMst}^{\mathrm{R}}$

adiuuantes ] abs. 32, [...]an[...] 64, cooperantes uel adiuuantes 77, adiurantes uel adiuuantes $\left(\mathrm{AMst}^{\mathrm{A}}\right)$, adiurantes $\mathrm{SPE}^{\mathrm{var}}$

autem ] abs. 32, lac. 67, om. AMst ${ }^{\mathrm{R}}$; + simul 61 $\mathrm{PEL}^{\mathrm{B}}$

et PEL var ] abs. 32, om. $51545^{8} \mathrm{AMst}^{\mathrm{AR}} \mathrm{PEL}^{\mathrm{A}} \mathrm{SPE}^{\mathrm{var}}$, lac. 67 ; + [ fratres] 32

exhortamur $\langle 32\rangle 547888$ ] obsecrantes $6177 \mathrm{PEL}^{\mathrm{B}}$, rogamus 64 , exhortantes

757689 , obsecramus AMsted, obsecramur AMstrar; + uos $\langle 32\rangle 54$

ne in uacuum gratiam dei $\langle 64\rangle$ ] lac. 32, ne in uacuum gratia dei 54677576 SPEvar

recipiatis ] [...]piatis 32 , suscipiatis 64 , excipiatis $7576 \mathrm{PEL}^{\mathrm{A}}$, accipiatis PEL $^{\text {var }}$ + uel excipiatis uos 77

6:2 Present: $3^{2} 51545^{8} 6164677576777884878889251$ AMst PEL CAr SPE ait ] ait enim in esaiam 58 , in esaia enim ait 61, lac. 64, om. $757677 \mathrm{AMst}^{\text {ed }}$ $\mathrm{PEL}^{\mathrm{var}}$, abs. 8487 251, dicit enim $\mathrm{AMst}^{\mathrm{R}}$, in esaia PEL ${ }^{\mathrm{var}}$, lac. CAr enim tempore $\langle 32\rangle\langle 64\rangle\langle 67\rangle]$ tempore $58 \mathrm{PEL}^{\mathrm{var}} \mathrm{CAr}$, tempore enim 61 (75) 7677 AMst $^{\text {ed }}$, abs. 8487251 , in tempore $\mathrm{AMst}^{\mathrm{R}}$; + inquit 617576 , + dicit 77 accepto ] acce[... 32 , acceptabili $61\langle 64\rangle 77 \mathrm{PEL}^{\mathrm{B}}$, lac. 67 , abs. 8487251 , oportuno CAr

exaudiui te ] lac. $3^{2}$ 67, abs. 8487 251, exaudiuit te PELA*

et in die salutis $\langle 32\rangle\langle 64\rangle$ ] lac. $67, a b s .8487251$, om. PEL ${ }^{\mathrm{var}}$, et dies salutis $\mathrm{CAr}$

adiuuaui te $89^{C *}$ ] lac. 32 , adiuui te $51545^{8} 6164757677788889^{*}$ AMst $\mathrm{PEL}^{\mathrm{AB}}$ SPE, lac. 67, abs. 8487251 , om. PEL ${ }^{\mathrm{var}}$; + fratres 8487251

ecce nunc tempus acceptabile $\left.\langle 32\rangle 6_{1}\langle 64\rangle\langle 251\rangle\right]$ lac. 67 , ecce nunc tempus acceptum $7576 \mathrm{CAr}$; + et 61

ecce ] abs. 84

nunc dies 87 ] lac. 32, abs. 84, nunc tempus uel dies $\mathrm{AMst}^{\mathrm{A}}$, dies $\mathrm{AMst}^{\mathrm{var}}$ salutis $\langle 64\rangle$ ] lac. 32 , abs. 84

6:3 Present: $3^{2} 5^{1} 545^{8} 616467757^{6} 7778878889251$ AMst PEL SPE

nemini dantes ullam $\langle 32\rangle\langle 67\rangle(87)\langle 251\rangle$ ] [nullam in quoq] uam dantes 64 , nullam in nullo dantes 7576 , ullam in nemine dantes 77 , in nullam in nullo dantes 89

offensionem $\langle 32\rangle$ ] lac. 64

ut non uituperetur $\langle 251\rangle]$ ut $\mathrm{n}[\ldots]$ 32, ut inreprehensible sit 61 $\mathrm{PEL}^{\mathrm{B}}$, lac. 64, ne uituperetur AMst

ministerium ] lac. 32 64; + nostrum $\left\langle 3^{2}\right\rangle\left(5^{1}\right) 545^{8} 6167757677788889251$ AMst PEL SPE 
6:4 Present: $3^{2} 5^{1} 545^{8} 616775767778878889$ 251 AMst PEL sPE sed in omnibus 54 ] lac. 32 , sed [...] 251

exhibeamus 5488 ] lac. 32, exhibuimus 61, ex[...] 67, commendantes 7576

89 AMst $^{\text {ed }}$, exhibentes 77, commendemus AMst ${ }^{\text {var }}$

nosmet ipsos ] lac. 32, [...] ipsos 67

sicut $\langle 32\rangle$ ] ut 61 AMst, ut uel sicut 77

dei ministros $\langle 32\rangle]$ dei ministri 7576 AMst; + uel ministri 77

in multa patientia 515488251 ] lac. $3^{2} 67$, in patientia multa 61757677

in tribulationibus $\left.\langle 32\rangle 88251 \mathrm{AMst}^{\mathrm{var}}\right]$ in pressuris $61 \mathrm{AMst}^{\mathrm{ed}}$, lac. 67

in necessitatibus $\langle 32\rangle$ ] lac. 67

in angustiis 61 ] lac. 3267

6:5 Present: $3^{2} 5^{1} 545^{8} 61677576777887888925^{1}$ AMst PEL sPE

in plagis ] lac. 3267

in carceribus $\langle 67\rangle$ ] lac. 32 , carceribus $\mathrm{PEL}^{\mathrm{B}}$ *

in seditionibus ] lac. $3^{2} 67$, in lacerationibus $61 \mathrm{PEL}^{\mathrm{B}}$, in iactationibus 89 , in perturbationibus AMst

in laboribus ] lac. 3267 , in tribulationibus 78

in uigiliis in ieiuniis 88 ] lac. $3^{2} 67$, in ieiuniis in ieiuniis $77^{*}$

6:6 Present: $3^{2} 5^{1} 545^{8} 616775767778878889$ 251 AMst PEL sPE

in castitate in scientia 54 ] lac. 3267 , in castitate $88^{*}$

in longanimitate $6 r \mathrm{AMst}^{\mathrm{R}}$ ] lac. $3^{2}$ 67, in magnanimitate $7576 \mathrm{AMst}^{\mathrm{ed}}$, om.

$88^{*}$, in magna animitate 89

\pm in beneuolentia 61 , in aequitate 89

in suauitate 87 ] lac. 32 67, in benignitate 757677 AMst

in spiritu sancto in caritate ] [...] in caritate 32 , lac. 67

non ficta ] lac. 3267 , non simulata AMst

6:7 Present: $3^{2} 5^{1} 545^{8} 6175767778878889$ 251 AMst PEL sPe

in uerbo ueritatis in uirtute dei ] lac. 32 , in uerbo ueritati dei $S P E^{\text {var }}$

per arma iustitiae a dextris $\langle 32\rangle 5^{1} 5458618788(89)$

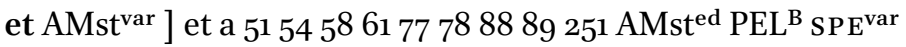

sinistris $\langle 32\rangle 87]+$ nostris 61

6:8 Present: $3^{2} 5^{1} 545^{8} 616775767778878889$ 251 AMst PEL sPE per gloriam ] lac. 3267

et ignobilitatem $\left\langle 3^{2}\right\rangle 87$ AMst $^{\text {var }}$ ] lac. 67 , in nouitatem 7576 , ignobilitatem $78^{*}$, et ignominiam $\mathrm{AMst}^{\mathrm{ed}}$, et nobilitatem $\mathrm{PEL}^{\mathrm{B}}{ }^{\mathrm{*}}$

per infamiam et bonam famam $\left.\left\langle 3^{2}\right\rangle\left\langle 25^{1}\right\rangle\right]$ lac. 67 , per infamia et bona 
famam 87 , per bonam et malam famem 89 , per infamiam sed bonam famam $\mathrm{AMst}^{\mathrm{R}}$

ut seductores ] lac. 67 , sicut seductores 89

et ueraces $\langle 67\rangle\langle 251\rangle]$ lac. 32 , sed ueraces $\mathrm{PEL}^{\mathrm{B}}$

6:9 Present: $3^{2} 5^{1} 545^{8} 616775767778878889$ 251 AMst PEL SPE sicut qui ] [...]ui 32, ut qui $61757677 \mathrm{PEL}^{\mathrm{B}}$, ut $\mathrm{AMst}{ }^{\mathrm{ed}}$, quasi $\mathrm{AMst}^{\mathrm{var}}$, et $\mathrm{AMst}^{\mathrm{var}}$, si qui PEL ${ }^{\text {var }}$

ignoti $87 \mathrm{PEL}^{\mathrm{var}}$ ] igno[...] 32, ignoremur 6189 , ignoramur $7576 \mathrm{PEL}^{\mathrm{B}}$, ignoramus uel ignoramur 77 , ignorati $\mathrm{PEL}^{\mathrm{A}}$

et $\mathrm{AMst}^{\mathrm{var}}$ ] lac. 32 , aut 58 , et qui $\mathrm{AMst}^{\mathrm{ed}}$, sed qui $\mathrm{AMst}^{\mathrm{R}}$

cogniti AMst ${ }^{\mathrm{var}}$ ] lac. $3^{2} 67$, ignoti $58^{*}$, cognoti $5^{8^{\mathrm{C}}} 87$, cognoscimur 61 $77^{\mathrm{C}} \mathrm{AMst}^{\mathrm{ed}} \mathrm{PEL}^{\mathrm{B}}$, cognoscimus $757^{6} 89$, cognoscemur $77^{*}$, cognoscamur $\mathrm{AMst}^{\mathrm{var}}$

quasi morientes $\left\langle 3^{2}\right\rangle$ ] lac. 67 , ut morientes 7576 AMst, sicut qui moriamur 89

et ecce uiuimus $\langle 32\rangle]$ [...] uiuimus 67 , sed ecce uiuimus $\mathrm{AMst}^{\mathrm{R}}$ ut $\langle 32\rangle]$ et 7576 , sicut qui 89 , quasi AMst

castigati $\left.\langle 67\rangle \mathrm{PEL}^{\mathrm{var}}\right][$ [... $] \mathrm{ti}$ 32, temptati $61757677 \mathrm{AMst} \mathrm{PEL}^{\mathrm{AB}}$, temptemur 89

et ] lac. 67 , ut 7576, om. 87 , sed AMst

non mortificati $\langle 32\rangle$ ] lac. 67 , morti non efficimur 89 , non morti traditi $\mathrm{AMst}^{\mathrm{ed}}$, morti non traditi AMst ${ }^{\mathrm{var}}$, morti traditi AMstrar

6:10 Present: $3^{2} 5^{1} 545^{8} 616775767778878889$ 251 AMst PEL SPE quasi tristes $\left.\langle 32\rangle{ }^{251}\right]$ ut tristes 7576 AMst, sicut tristes 89 semper autem gaudentes $\langle 87\rangle$ ] lac. 67 , semper aut gaudentes 88 sicut ] lac. 67, sicut et 757689 , ut AMst egentes $\langle 32\rangle]$ egeni 6177 , pauperes $\mathrm{AMst}^{\mathrm{R}}$ multos autem ] mult[2] autem 32 , multo autem 67 , sed multos 89 , multos enim $\mathrm{AMst}{ }^{\mathrm{AD}}$

locupletantes 3287 ] ditantes AMst tamquam $\langle 32\rangle 7778$ ] ut 61 AMst, quasi $\mathrm{PEL}^{\mathrm{B}}$ nihil habentes 51545887

et omnia possidentes $326_{1}\langle 67\rangle 87$ ] omnia autem possidentes AMst ${ }^{\text {ed }}$, et omnia autem possidentes $\mathrm{AMst}^{\mathrm{var}}$, omnia possidentes $\mathrm{SPE}^{\mathrm{var}}$

6:11 Present: 5154586167757677788889 251 AMst PEL os nostrum patet ad uos

o $\mathrm{AMst}^{\mathrm{var}}$ ] om. $5^{1} 757^{6} \mathrm{AMst}^{\mathrm{ed}}$ 
corinthii cor nostrum $586177^{*} 88$

dilatatum est ] dilatum est $54^{*}$

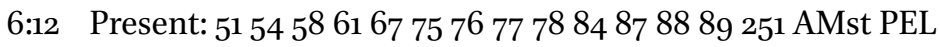

\pm fratres 8487

non angustiamini 84$]$ non angustemini 54 , non an[...] 67

in nobis ] lac. 67, abs. $84 ;+\operatorname{sed} 51$

angustiamini 54 ] coartamini $61 \mathrm{PEL}^{\mathrm{B}}$, abs. 84

autem ] om. 51 AMst ${ }^{\text {var }}$, abs. 84

in uisceribus uestris $\left(54^{*}\right)$ ] abs. 84 , in uisceribus nostris $88^{*} \mathrm{AMst}^{\mathrm{var}}$

6:13 Present: 515458616775767778878889 251 AMst PEL SPE

eandem autem habentes ] eandem habentes $61 \mathrm{AMst}_{\mathrm{PEL}}{ }^{\mathrm{B}}$, eandem autem

75767789 , abs. SPE

remunerationem ] mercedis remunerationem 58 , mercedis retributionem

61 $\mathrm{AMst}^{\mathrm{ed}}$, mercedem retributionis AMst ${ }^{\mathrm{var}}$, abs. SPE

tamquam $78 \mathrm{AMst}^{\mathrm{R}}$ ] sicut $6177 \mathrm{AMst}^{\mathrm{ed}}$, abs. SPE

filiis dico ] fidelis dico $87, a b s$. SPE

dilatamini et uos $\langle 67\rangle 87\langle 251\rangle$

6:14 Present: 32515458616775767778878889251 AMst PEL tes SPE

\pm uos autem fratres 32 , et $5^{1} 5^{8} 61757677788788^{*} 89{ }_{251}$ AMst $^{\text {ed }}$ PEL ${ }^{A B}$ SPE var $^{\text {va }}$

nolite ] non uel ne 77

iugum ducere $\left.\left\langle 3^{2}\right\rangle\right]$ sitis subiugati uel subiugantes uel esse 77 , coniungi TES $^{\text {ed }}$, iungi TES ${ }^{\text {var }}$

cum infidelibus $\left(78^{*}\right)$ ] cum fidelibus AMst ${ }^{\text {var }}$

quae enim $\left\langle 3^{2}\right\rangle 58617787 \mathrm{TES}^{\mathrm{var}}$ ] quae autem ${ }_{51} \mathrm{AMst}^{\mathrm{var}} \mathrm{TES}^{\text {ed }}$, quae 54

participatio 251 ] portio $61 \mathrm{AMst}^{\mathrm{R}}$; + enim 54 , + est $61 \mathrm{TES}^{\mathrm{ed}}$, + uel portio 77 iustitiae 515458768788 ] luminis TE Svar $^{\text {va }}$

cum iniquitate (87) $\mathrm{AMst}^{\mathrm{AR}} \mathrm{TES}^{\mathrm{var}}$ ] et iniquitati $\mathrm{AMst}^{\mathrm{ed}} \mathrm{TES}^{\mathrm{ed}}$, cum tenebrae TES ${ }^{\text {var }}$, cum iniquitatis TES ${ }^{\text {var }}$

aut quae $\left.\left\langle 3^{2}\right\rangle 515458617787 \mathrm{AMst}^{\mathrm{var}}\right]$ et quae AMsted, uel TES $\mathrm{S}^{\mathrm{var}}$

societas $\left.\left\langle 3^{2}\right\rangle\right]$ communio TES $S^{\text {ed }}$, conuentio TES ${ }^{\mathrm{var}}$, communicatio TES ${ }^{\mathrm{var}}$; + est TES $S^{\text {ed }}$

luci TES ${ }^{\text {var }}$ ] lucis $3^{2} 757687 \mathrm{PEL}^{\mathrm{var}}$, lucis uel luci 77 , luminis $89 \mathrm{AMst}^{\mathrm{var}}$, luce 251, lumini AMsted ${ }^{\text {TES }} S^{\text {ed }}$, lucit PEL ${ }^{\text {var }}$, om. TES ${ }^{\text {var }}$

ad tenebras ] cum tenebris $61 \mathrm{AMst}^{\mathrm{ed}} \mathrm{PEL}^{\mathrm{var}}$, tenebris AMstvar, a tenebris PEL $^{\text {var }}$, et tenebrarum TES ${ }^{\text {var }}$, om. TES ${ }^{\text {var }}$ 
6:15 Present: $3^{2} 51545^{8} 616775767778878889251$ AMst PEL TES $^{\dagger}$ SPE quae autem $\left.51545^{8} 6177_{7}\left\langle 87^{C}\right\rangle\right]$ autem $87^{*}$, quaeue AMsted, quae uel AMst ${ }^{\text {tar }}$, quae $\mathrm{AMst}^{\mathrm{var}}$, quid autem PEL ${ }^{\mathrm{var}}$

conuentio ] communicatio 61 , conuenientia uel communicatio 77 , consensio AMst, contentio $\mathrm{SPE}^{\mathrm{var}}$

christi AMst ${ }^{\mathrm{var}} \mathrm{TES}^{\mathrm{var}}$ ] in christo 77 , christo AMst ${ }^{\mathrm{ed}}$

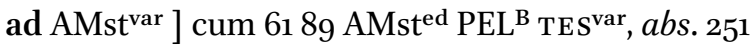

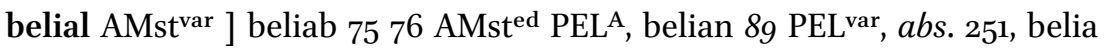
AMst $^{\text {var }}$, belzebub TES ${ }^{\text {var }}$

aut quae pars $515458676777(87) \mathrm{AMst}^{\mathrm{R}}$ ] lac. 251, aut quae participatio AMsted

fideli ] fidelium 87 , abs. 251, fidelibus $\mathrm{SPE}$ var

cum infidele ] cum infideli $5^{1} 5^{8} 61767789$ AMst $^{\text {var }}$ PEL $^{\mathrm{B}}$ SPE ${ }^{\text {ed }}$, abs. 251, cum infidelibus PEL ${ }^{\text {var }}$, infidele SPEvar

6:16 Present: $3^{2} 5^{1} 545^{8} 616775767778878889$ AMst PEL sPe

qui ] quis $54 \mathrm{PEL}^{\mathrm{B}}$, quis uel quae 77 , quae $88^{*} 89$, uel quae $\mathrm{AMst}$, quid $P E L^{\text {var }}$ autem ] om. AMst

consensus ] consensus uel consensio 77, conuentio AMst

templo ] templi 88 AMst $^{\text {var }}$

dei cum idolis $\left.\langle 32\rangle 51545861 \mathrm{PEL}^{\mathrm{var}}\right]$ cum idolis PEL ${ }^{\mathrm{var}}$

uos ] nos 7576

enim ] autem 87 PEL $^{\text {var }} \mathrm{SPE}^{\mathrm{var}}$, om. PEL var

estis templum dei uiui $\langle 32\rangle]$ templum dei sumus uiui 7576 , templum dei estis uiui 7789 AMst $^{\text {ed }}$, templum estis dei uiui AMst ${ }^{\text {var }}$, estis templo dei uiui SPEvar

sicut dicit $\left.\left\langle 3^{2}\right\rangle\right]$ sicut dixit 5467 AMst, dicit enim 6175767789

deus ] scriptura 61, dominus $\mathrm{AMst}^{\mathrm{A}} \mathrm{PEL}^{\text {var }} \mathrm{SPE}$

quoniam ] lac. 67, abs. 87, quia $\mathrm{AMst}^{\mathrm{A}}$

inhabitabo $\langle 32\rangle$ ] lac. $67, a b s .87$, habitabo 89 AMst $^{\text {var }}$ PEL $^{\text {var }}$

in illis et $\langle 32\rangle]$ in eis et 61 , lac. 67, abs. 87

inambulabo ] in eis ambulabo 51 , ambulabo 58 , inter eos ambulabo $\langle 32\rangle 61$ 757677 AMst $^{\text {ed }}$, lac. 67, abs. 87, inter illos ambulabo 89, inter eos inambulabo AMst ${ }^{\mathrm{var}} \mathrm{SPE}^{\mathrm{var}}$, intra eos ambulabo $\mathrm{PEL}^{\mathrm{B}}$, inambulabo inter eos SPEvar

et ero illorum deus $\langle 32\rangle]$ lac. 67 , et ero ipsis uel illis deus 77 , abs. 87

$\dagger{ }_{2}$ Cor 6:15. In TES, the words christi cum belzebub from this verse are found in a variant to the quotation of 6:14, but the rest is not cited. 
et ipsi $\langle 32\rangle$ ] lac. $67, a b s .87$, et ipse 88

erunt mihi populus $\left\langle 3^{2}\right\rangle 5^{8}$ ] lac. 67 , abs. 87 , erit mihi populus 88 , mihi erunt populus AMstrar, erant mihi populus PEL ${ }^{\text {var }}$

6:17 Present: $3^{2} 5^{1} 545^{8} 61\langle 67\rangle^{\dagger} 757^{6} 77788889$ AMst PEL SPE propter quod $\left.\langle 32\rangle\left(78^{*}\right)\right]$ quapropter $\mathrm{AMst}^{\mathrm{R}}$

exite de medio eorum AMst ${ }^{\mathrm{var}}$ ] lac. 32, exite de medio illorum 61 $\mathrm{AMst}^{\mathrm{ed}}$ et separamini dicit dominus $\left.\left\langle 3^{2}\right\rangle 6_{1}\right]+$ uos qui portatis uasa domini 89 et inmundum 76 ] inmun [dum] 32 , et ab inmundo 77 ne tetigeritis $\left(54^{*}\right)$ ] lac. 32 , ne tangamini 77 , nolite tangere AMst, non tetigeritis PEL var

6:18 Present: $3^{2} 5^{1} 545^{8} 61\langle 67\rangle^{\dagger} 757^{6} 777888$ 89 AMst PEL SPE et ego recipiam uos et $\langle 32\rangle$ ] et ego 51, et ego suscipiam uos et 6177 AMst ero uobis in patrem $\left\langle 3^{2}\right\rangle$ ] ero uobis in patre 7788 et uos eritis mihi $\left.\langle 32\rangle 545^{8}\right]$ et eritis mihi $\mathrm{SPE}^{\mathrm{var}}$ in filios $\langle 32\rangle]$ in filiis 54 et ] + in 89 filias ] filiabus 54 dicit dominus omnipotens $(88)]$ di $[\ldots] 3^{2}$

7:1 Present: $3^{2} 5^{1} 545^{8} 6167757677788889$ AMst PEL CAr SPE has ] lac. 32, haec 6 AMst $^{\text {ed }}$, hac 75 76, hoc AMstrar igitur $\langle 32\rangle$ ] ergo 61 AMst, igitur uel ergo 77 habentes promissiones ] ha[...] 32, repromissiones habentes 58 , promissa habentes 61, habentes repromissiones 77, habentes promissa AMst carissimi $517778 \mathrm{AMst}^{\mathrm{var}}$ ] lac. 32, fratres carissimi AMst ${ }^{\mathrm{ed}}$ mundemus ] lac. 32, emundemus 58 nos $\mathrm{AMst}^{\mathrm{var}}$ ] lac. 32 , nosmetipsos $\mathrm{AMst}^{\mathrm{ed}}$, nos ipsos $\mathrm{AMst}^{\mathrm{R}}$ ab omni inquinamento carnis (61) ] lac. 32 et spiritus ] lac. 32, om. 54 AMst, abs. CAr perficientes ] lac. 32, ut perficiamus $\mathrm{AMst}^{\mathrm{R}}$, abs. CAr sanctificationem ] lac. 32 , sanctitatem $\mathrm{AMst}^{\mathrm{ed}}$, sanctitates $\mathrm{AMst}^{\mathrm{A}}$, abs. CAr; + spiritus 5461 AMst PEL $^{\mathrm{B}}$ in timore dei ] lac. 32 , in timorem dei $8889 \mathrm{SPE}^{\mathrm{var}}, a b s$. CAr

$\dagger{ }_{2}$ Cor. 6:16-18. The quotation in VL 67 appears to have been written in red ink and is now illegible. 


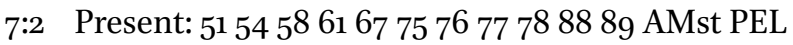

capite nos 75 ] capite uos $54^{*}$, capaces estote nostri AMst

neminem ] nemini AMst ${ }^{\text {var }}$

laesimus 515458677888 ] nocuimus 6175767789 AMst PEL $^{\mathrm{B}}$

neminem

corrupimus $\mathrm{AMst}^{\mathrm{R}}$ ] corrumpimus $\langle 54\rangle 5^{8} 617576 \mathrm{PEL}^{\mathrm{A}}$, grauauimus AMst $^{\text {ed }}$, corripuimus PEL ${ }^{\text {var }}$

neminem circumuenimus

7:3 Present: 51545861677576777888 89 AMst PEL

non ad condemnationem 51586188 ] + uestram 5458 , + uestri hoc $\mathrm{AMst}^{\mathrm{R}}$

dico praedixi 515461677788 ] dico praediximus $58 \mathrm{PEL}^{\text {var }}$

enim ] + uobis $58,+$ iam uobis $\mathrm{AMst}^{\mathrm{R}}$

quod $\left.\mathrm{AMst}^{\mathrm{R}}\right]$ quia AMst ${ }^{\text {ed }}$

in cordibus nostris ] in cordibus uestris $77^{*}$

estis (88) ] om. PEL var

ad conmoriendum 515461677576777888

et ad ] et $547788^{*}$ PEL $^{\text {var }}$, ad 67

conuiuendum $88^{*}$ ] conuiuentium 54 *

7:4 Present: $5^{1} 545^{861} 67757677788488$ 89 AMst PEL

multa ] om. $54^{*}$, fratres multa 84

mihi fiducia 58 ] om. $54^{*}$, mihi libertas $84 \mathrm{PEL}^{\mathrm{B}}$

est ] om. $5154^{*} 61757677788489$ AMst PEL $^{\mathrm{B}}$

apud uos $\left.5158 \mathrm{AMst}^{\mathrm{AR}}\right]$ om. $54^{*}$, ad uos $7576778488^{*} 89 \mathrm{AMst}^{\mathrm{ed}} \mathrm{PEL}^{\mathrm{B}}$; + est 51757689

multa mihi gloriatio $545^{8}$ ] multa gloriatio 7576 , abs. 84

pro uobis ] abs. 84, de uobis AMst ${ }^{\mathrm{R}} \mathrm{PEL}^{\mathrm{var}}$; + est 61

repletus sum ] abs. 84

consolatione 61 AMst $^{\text {var }}$ ] exhortatione 7576 , abs. 84, exhortationem 89, consolationem AMsted

superabundo gaudio 515889 AMst $\left.^{\text {ed }}\right]$ semper abundo gaudio 61 AMst ${ }^{\mathrm{var}}$, abs. 84

in omni $\mathrm{AMst}^{\mathrm{R}}$ ] lac. 67, in multa $757689 \mathrm{PEL}^{\mathrm{B}}$, abs. 84, super omni AMst $^{\text {ed }}$

tribulatione $\left(78^{*}\right)$ ] pressura 61 AMst, lac. 67 , abs. 84

nostra ] uestra $5^{16} 1^{\mathrm{C}}$, lac. 67, abs. 84 


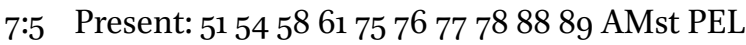

nam et cum ] nam cum $61{ }_{1}$ PEL $^{\text {var }}$, etenim $7788^{*}$, nam cum et 78 , etenim cum AMst

uenissemus 89 ] uenissem 51 , conuenissemus 61 , uenientibus nobis $7788^{*}$ macedoniam $88^{c} \mathrm{PEL}^{\mathrm{var}}$ ] in macedoniam $6175767788^{*} 89 \mathrm{AMst}^{\mathrm{var}} \mathrm{PEL}^{\mathrm{A}}$, in macedonia PEL ${ }^{\mathrm{B}}$

nullam

requiem habuit ] requiem inuenit 54 , requiem habunt 7576 , habuit requiem 89

caro nostra sed

omnem ] omnem in omnibus 58 , in omnibus $6175767788^{*} 89$ AMst PEL ${ }^{\mathrm{B}}$, omni $88^{\mathrm{C}}$, in omni PEL ${ }^{\text {var }}$

tribulationem passi ] sumus adflicti $61 \mathrm{AMst}^{\mathrm{ed}} P E L^{B}$, tribulatio 7576 , tribulationibus passi $88^{*}$, tribulatione passi $88^{\mathrm{C}} \mathrm{PEL}^{\mathrm{var}}$, adflicti fuimus $A M s t^{R}$, tribulationibus positi PEL ${ }^{\text {var }}$; sumus $545^{8}$

foris pugnae 51545888 ] foris pugna PEL var

intus ] intus autem 61, et intus AMst ${ }^{\text {var }} \mathrm{PEL}^{\text {var }}$ timores ] + uel formidines 77

7:6 Present: 51545861757677788889 AMst PEL sed ] + deus $58,+$ is 88

qui consolatur humiles 61 ] + et 51 , + deus AMstvar consolatus est $6 r$ nos deus ] nos AMstrar, et nos deus PEL ${ }^{\text {var }}$ in aduentu ] in praesentia $6188^{*}$, in aduentum $757689 \mathrm{AMst}^{\mathrm{var}} \mathrm{PEL}^{\mathrm{B}}$; + uel praesentia 77 titi 545888 ] suo 51

7:7 Present: $51545^{8} 61757^{6} 77788889$ AMst PEL non solum autem $\mathrm{AMst}^{\mathrm{A}}$ ] non solum $5^{1} 77$ 89, et non solum AMsted in aduentu ] in aduentum $54757689 \mathrm{AMst}^{\mathrm{var}} \mathrm{PEL}^{\mathrm{B}}$, in praesentia uel in aduentu 77 , in praesentia $88^{*}$, om. PEL var eius sed etiam AMst ${ }^{\text {var }}$ ] eius sed et 7789 AMst $^{\text {ed }}$, eius sed $88^{*}$ AMst $^{\text {var }}$ in ] om. 61 solacio $88^{C}$ ] consolatione $54777888^{*} \mathrm{AMst}^{\mathrm{PEL}} \mathrm{L}^{\mathrm{B}}$, solacione 58 , consolatio 61, consolationem 757689 quo ] qui 51 , qua $587576777^{8} 88^{*} 89 \mathrm{AMst}$, quia $\mathrm{PEL}^{\mathrm{B}}$, quod $\mathrm{PEL}^{\text {var }}$ consolatus est 61 ] consolatus $\mathrm{PEL}^{\mathrm{B}}$; + ipse $\mathrm{AMst}^{\mathrm{R}}$ in uobis ] in nobis AMst ${ }^{\text {var }} \mathrm{PEL}^{\mathrm{var}}$ referens ] nuntians 61757677 , adnuntians $88^{*}$ AMst 
nobis ] uobis PEL var

uestrum desiderium 61 ] uestrum fletum $\mathrm{PEL}^{\mathrm{B}}$; + uel maerorem 77

uestrum fletum ] uestrum maerorem $7576 P E L^{B}$, uestrum memorem $88^{*}$, uestrum desiderium PEL var

uestram aemulationem $\langle 51\rangle 54586177>888$

pro me ita ut magis ] ita magis $\mathrm{PEL}^{\mathrm{var}}$

gauderem ] gaudendum sit 89

7:8 Present: 515458617576777888 89 AMst PEL SPE

quoniam et si ] quia et si $88 \mathrm{AMst}^{\text {ed }}$, si autem 89 , unde et si $\mathrm{AMst}^{\mathrm{R}}$, quoniam et SPEvar

contristaui uos in epistula ${ }_{51} 617677788889$ ] + mea 75767778 , + prima PEL ${ }^{\text {var }}$

non me paenitet 515458617888 ] non paenitet me 77 , non me paeniteret AMst $^{\text {var }}$

et si $(77)$

paeniteret 5154587788 ] paeniteret me 61 , paenitet $7576 \mathrm{SPE}^{\mathrm{var}}$, me paeniteret 78 , me paeniterit $89^{*}$, paeniterit $89^{\mathrm{C}}$, paenituisset $\mathrm{AMst}^{\mathrm{R}}$, paenitere AMst $^{\text {var }}$

uidens $\mathrm{AMst}^{\mathrm{R}}$ ] uideo $61757^{6} 777^{8} 89 \mathrm{AMst}^{\mathrm{ed}} \mathrm{PEL}^{\mathrm{B}}$, uidet $88^{*}$

quod ] quoniam 51, quia $61 \mathrm{AMst}^{\mathrm{var}} \mathrm{PEL}^{\mathrm{B}}$, enim quod $777^{8} 88^{\mathrm{C}}$, quia et $\mathrm{AMst}^{\mathrm{ed}}$, quod et $\mathrm{AMst}^{\mathrm{R}}$

epistula illa et si ${ }_{51} 617677788889$ AMst $^{\mathrm{R}}$ ] illa epistula et si $A M t^{e d}$

ad horam uos contristauit 54 ] ad horam contristauit uos $75768889 \mathrm{PEL}^{\mathrm{B}}$, contristauit uos ad horam AMst ${ }^{\mathrm{ed}}$, contristauit ad horam AMst ${ }^{\mathrm{AD} *}$

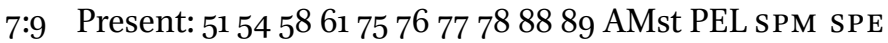

nunc gaudeo ] et nunc gaudeo 58 , gaudeo 7576 , nunc autem gaudeo $S P E^{\text {var; }}$

+ gaudeo autem $61 \mathrm{PEL}^{\mathrm{B}}$

non quia contristati estis sed

quia contristati estis ] contristati estis 89

ad $\left.\mathrm{AMst}^{\mathrm{AR}}\right]$ in ad 77, om. $88^{*}$, in $\mathrm{AMst} \mathrm{ed}^{\mathrm{ed}}$

paenitentiam $5154586176777888^{\circ}$ ] om. $88^{*}$

contristati enim estis ] nam contristati estis $61 \mathrm{PEL}^{\mathrm{B}}$, contristati estis enim

77, om. $88^{*}$, contristati enim AMst ${ }^{\mathrm{var}}$, contristati estis $\mathrm{SPE}^{\mathrm{var}}$; + ad paenitentiam $88^{\mathrm{Cl}}$ (om. $\left.88^{\mathrm{C} 2}\right)$

secundum deum ] deum secundum 54 , coram deo 89

ut in nullo ] om. 88, ut in illum 89, ita ut in nullo $\mathrm{AMst}^{\mathrm{R}}$, ut nullo $\mathrm{SPE}^{\mathrm{var}}$

detrimentum ] om. 88 , detrimento PEL ${ }^{\mathrm{A} *}$

patiamini ex nobis ] patiamini a nobis 51 , om. 88, patiemini ex nobis $\mathrm{PEL}^{\mathrm{var}}$ 
7:10 Present: 51545861647576777888 89 AMst PEL SPM SPE quae enim $5154586188^{c}$ ] lac. 64 , nam 75767789 SPM, om. $88^{*}$ secundum deum ] lac. 64, secundum $78^{*}$, om. $88^{*}$

tristitia est ${ }_{51}{ }_{54} \mathrm{PEL}^{\mathrm{var}}$ ] lac. 64, tristitiam 7576 , tristitia $7788^{*} 89$ SPM, est tristitia AMst PEL ${ }^{\mathrm{AB}}$

paenitentiam 5154586176777888 ] lac. 64

in salutem stabilem operatur ] lac. 64 , in salutem inpenitendam operatur $88^{*}$, ad salutem stabilem operatur AMst, stabilem in salutem operatur SPEvar

saeculi autem tristitia $51545^{8}$ ] saeculi enim tristitia 61 , [...]am 64, nam huius saeculi tristitia 7576 , mundi uel saeculi autem tristitia 77 , mundi autem tristitia 88 , huius autem mundi tristitia 89 , tristitia enim huius mundi AMst ${ }^{\mathrm{ed}}$, tristitia uero huius mundi $\mathrm{AMst}^{\mathrm{AD}}$, tristitia autem huius mundi AMst ${ }^{\mathrm{var}}$, saeculi autem tristitiam PEL ${ }^{\mathrm{var}}$, nam huius mundi tristitia SPM

mortem operatur ] morte operatur 89

7:11 Present: $5^{1} 545^{8} 61647576777888$ 89 AMst PEL sPE ecce enim (75) ] et enim SP Evar

hoc ] in hoc 61 , id $6488^{*}$

ipsum ] ipsud $89 ;+$ quod 78

secundum deum ] secundum dominum $88^{*}$

contristari ] contristaui $78 \mathrm{PEL}^{\mathrm{var}} \mathrm{SPE}^{\mathrm{var}}$, contristare AMst ${ }^{\mathrm{var}} \mathrm{PEL}^{\mathrm{var}}$

uos ] om. $647788 * 89$ AMst

quantam ] quantum $89 \mathrm{AMst}^{\text {var }} \mathrm{PEL}^{\mathrm{B}} \mathrm{SPE}^{\mathrm{var}}$, quanta $\mathrm{AMst}^{\mathrm{var}}$

in uobis operatur ] perfecit uobis 64 , operatus est uobis 7576 , operatus est in uobis 77, [5-6] in uobis $88^{*}$, operatus est nobis 89, efficit in uobis AMsted $\mathrm{PEL}^{\mathrm{B}}$, perfecit in uobis AMstvar, perficit in nobis $\mathrm{AMst}^{\mathrm{var}}$

sollicitudinem $5^{8}$ ] industriam 64 , lac. $88^{*}$

sed defensionem ] sed excusationem sed defensionem $54^{*}$, sed excusationem $61757677 \mathrm{AMst}^{\mathrm{ed}}$, sed ex curationem 64, lac. 88*, sine excusatione $\mathrm{AMst}^{\mathrm{A}}$, non excusationem $\mathrm{AMst}^{\mathrm{R}}$, abs. SPE

sed indignationem $\mathrm{AMst}^{\mathrm{DR}}$ ] sed refectionem 64, lac. 88* om. AMst ${ }^{\mathrm{ed}}$, abs. SPE

sed timorem $\langle 64\rangle]$ abs. SPE

sed desiderium 61 ] om. 58 , sed desiderio AMst ${ }^{\mathrm{var}}$, abs. SPE; + nostrum PEL var sed aemulationem sed uindictam in omnibus $5154 \quad 586178$ 88 ] sed aem $[u]$ lationem sed uindicta in omnibus $64, a b s$. SPE

exhibuistis uos 5475$]$ c $[\mathrm{om}]$ mendatis uos 64 , lac. $88^{*}$, castos uos exhibuistis AMst, abs. SPE 
incontaminatos esse 54 ] sinceres esse $61 \mathrm{PEL}^{\mathrm{B}}$, castos esse 64757689 , incontaminatos uel castos esse 77 , lac. $88^{*}$, esse AMst, abs. SPE

negotio 51 ] re ipsa 64 , in negotio 7576 , lac. $88^{*}$, in eo negotio $\mathrm{AMst}^{\mathrm{R}}, a b s$. SPE

7:12 Present: $51545^{8} 61647576777888$ 89 AMst PEL CAr

igitur et si ] igitur et 58 , igitur si 757689

scripsi uobis $64 \mathrm{AMst}^{\mathrm{var}}$ ] scripsi $757689 \mathrm{AMst}^{\mathrm{ed}} \mathrm{PEL}^{\mathrm{A}}\langle\mathrm{CAr}\rangle$

non propter eum ] nec propter eum 76

qui fecit iniuriam 78 ] qui iniuriam fecit $5^{1} 6175^{*} 7677\left(\mathrm{PEL}^{\mathrm{B}}\right) \mathrm{CAr}$, qui tamen

fecit iniuriam 58 , qui iniquitatem fecit 64 , qui iniuriam passus est $75^{\mathrm{C}}$, qui iniuriam fẹçịt $75^{\mathrm{C} 2}$, iniuriatorem $78^{\mathrm{gl}}$, qui $[\ldots] 88^{*}$, qui inique uersatus est AMst; + uel non propter iniuriatorem $77,+$ sed AMst ${ }^{\text {ed }},+$ tantum PEL var nec ] neque $\langle 64\rangle 777888^{*}$ AMst $\mathrm{PEL}^{\mathrm{B}}$, ne PEL ${ }^{\mathrm{var}}$, abs. CAr propter eum ] propter eum [4-8] 88*, propter ipsum PEL ${ }^{\text {var }}$, abs. CAr qui ] quia 58 , qui [4-6] $88^{*}$, lac. CAr \pm iniuriam $6175767778 \mathrm{PEL}^{\mathrm{B}}$, iniquitatem 64, inique AMst passus est sed ] tractatus est sed AMst, sed PEL ${ }^{\text {var }}$, abs. CAr ad manifestandam 78 ] propter manifestandam $64777^{\mathrm{gl}} 88^{*}$, ut manifestetur $757689 \mathrm{AMst}^{\mathrm{AD} *}$, ut manifestaretur AMst ${ }^{\mathrm{ed}}$, abs. CAr

sollicitudinem nostram $5861\left(88^{\mathrm{C}}\right)$ ] industriam uestram 64 , sollicitudo nostra $75^{*} 89$ AMst, sollicitudo uestra $\left(75^{\mathrm{C}}\right) 76$, soll[8] nostram $88^{*}$, abs. CAr

quam ] om. $6488 * 89$, quae est 757677 , qui PEL ${ }^{\text {var }}$, quia PEL ${ }^{\text {var }}$, abs. CAr pro uobis habemus ] pro nobis $\langle 64\rangle 757677$, pro uobis $88^{*} 89$, abs. CAr ad uos ] om. $5^{1} 6189$ AMst PEL $^{\mathrm{A}}$, abs. CAr coram deo ] coram domino $88^{*}$, om. PELA, abs. CAr

7:13 Present: $51545^{8} 61647576777888$ 89 AMst PEL ideo ] in deo 89; + et $546177 \mathrm{AMst}^{\text {ed }} \mathrm{PEL}^{\mathrm{B}},+$ autem $5^{8}$ consolati sumus $61(88)$ ] consolationem accepimus AMst ${ }^{\text {ed }}$, consolationem accipimus AMst ${ }^{\text {var }}$

in consolatione autem 61 ] in consolationem autem $64 \mathrm{AMst}^{\mathrm{var}}$ nostra ] nostram $\langle 64\rangle \mathrm{AMst}^{\mathrm{var}}$, uestra PEL var abundantius $51545^{86188^{C}}$ ] plus 64, [2-4] 88*, magis AMst; + autem 5489 magis ] om. 89, magisque AMst gauisi sumus $61(88)$ ] gaudio repleti sumus $\mathrm{AMst}^{\mathrm{R}}$ super ] in $6488^{*}$ gaudium AMst ${ }^{\text {var }}$ ] gaudio $648889 \mathrm{AMst}^{\mathrm{ed}} \mathrm{PEL}^{\mathrm{B}}$, consolatione $\mathrm{AMst}^{\mathrm{R}}$ titi quia 5888 ] quia 5 , titi quoniam 7576 
refectus est ] perfectus est 7576 , requieuit AMst, profectus est $P E L^{B}$ spiritus eius ] spiritus $88^{*} \mathrm{PEL}^{\text {var }}$, spiritus meus AMstrar ab omnibus uobis ] in omnibus uobis AMst

7:14 Present: 51545861647576777888 89 AMst PEL

et si quid 54 ] quoniam si quid $647788^{*} 89 \mathrm{PEL}^{\mathrm{B}}$, quia si quid 7576 , quoniam $\mathrm{AMst}^{\mathrm{ed}}$, et quoniam $\mathrm{AMst}^{\mathrm{R}}$ apud $51545^{8} 75$ ] om. $647788^{*}$, in AMsted, cum AMstvar illum ] illi 64, ei 77, quibuscumque AMst; + gloriatus sum 6177 de $\mathrm{AMst}^{\mathrm{R}}$ ] pro AMsted uobis ] + in ueritate $61 \mathrm{PEL}^{\mathrm{B}}$ gloriatus sum ] locutus sum 61, om. 77 non sum confusus 61 ] non erubui 64 , non spiritu confusus 89 , non [...] 88* sed sicut ] sed quomodo 64 , sed quemadmodum AMst, lac. 88*; + uobis 64 omnia ] + uel omnino 77

uobis ] de uobis $5^{1} 58,[1]$ uobis $54^{*}$, om. 616477

in ueritate locuti sumus (88) 89 ] in ueritate locutus sum PEL ${ }^{\text {var; }}$ + de uobis $61,+$ uobis 77

ita et gloriatio 88 ] ita et gloria 5477 nostra ] uestra AMst ${ }^{\text {var }}$

quae fuit ad titum $51545^{8} 6177^{c} 88$ ] quae ad titum fuit 757689 , quae ad titum $77^{*}$, apud titum AMst, quae fuit de tito PEL var ueritas facta est

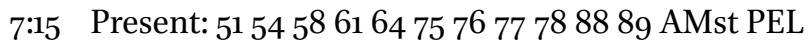
et uiscera eius ] et uiscera illius AMst

abundantius in uos PEL ${ }^{\text {var }}$ ] abundantius in uobis $51545861647576777^{8}$ 88 AMst PEL ${ }^{\mathrm{AB}}$

sunt ] sint AMst ${ }^{\mathrm{var}}$

reminiscentis ] reminiscens $61 \mathrm{PEL}^{\mathrm{A}}$, rememorantes 757689 , reminiscentes 88 AMst $^{\mathrm{AD}}$ PEL $^{\text {var }}$

omnium uestrum ] uestram 89, omnium uestri $\mathrm{AMst}^{\mathrm{R}}$, omnium nostrum AMst $^{\text {var }}$

oboedientiam $51545^{8} 6188$ ] obaudientiam 7576 AMst PEL ${ }^{\mathrm{B}}$, oboeditionem 77 , oboedientium 78

quomodo ] quam 89 cum timore et tremore $(75)(76)]$ cum timore (89)

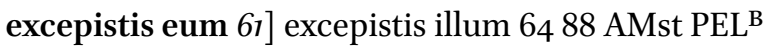


7:16 Present: 51545861647576777888 89 AMst PEL

gaudeo quod in omnibus

confido in uobis $\left(61^{*}\right)$ ] confido uobis 51 , confido de uobis $6488^{*}$, uobis confido 89, uobis PEL var

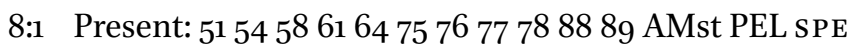

notam autem ] notescimus autem 77 , notum autem $89 \mathrm{AMst}^{\mathrm{A} * \mathrm{D} *}$

facimus uobis $\langle 64\rangle \mathrm{PEL}^{\mathrm{var}}$ ] fecimus 51 , uobis facimus $6188 \mathrm{PEL}^{\mathrm{AB}} \mathrm{SPE}$, uobis

77 , uobis facio 89 , uobis fecimus PEL var

fratres $\langle 64\rangle]$ om. AMst ${ }^{\text {var }}$

gratiam dei ] gratia domini 89, gratiam $\mathrm{AMst}^{\mathrm{var}}$, gloriam domini $\mathrm{PEL}^{\mathrm{B}}$

quae data est 5154586188 ] datam 77 ; + nobis PEL ${ }^{\text {var }},+$ mihi SPEvar in ] om. PEL var

ecclesiis macedoniae 51545861647576788889

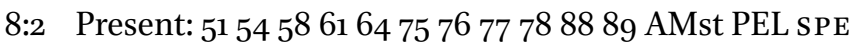

quod in ] quod $61 \mathrm{SPE}^{\mathrm{var}}$, quia in $64, \mathrm{qu}[2]$ in $88^{*}$, quoniam in PEL var

multo experimento $\left.\left(89^{*}\right)\right]$ experimento 61, multam $\mathrm{p}[$ rob]ationem 64 , multa probatione $75767788^{*}$ AMst; + et 89

tribulationis ] lac. 64, tribulatione 89, pressurae AMst; + uel tribulatione 77 abundantia gaudii 51545861 ] lac. 64, abundat gaudium AMst, abundantiae gaudii $S \mathrm{PE}^{\mathrm{var}}$

ipsorum ] lac. 64, eorum $757688 * 89$; + fuit 58 PEL $^{\mathrm{B}}$

et $\mathrm{AMst}^{\mathrm{R}} \mathrm{SPE}^{\mathrm{var}}$ ] lac. 64, et quod $\mathrm{AMst}^{\mathrm{ed}}$, om. $\mathrm{SPE}^{\mathrm{ed}}$

altissima paupertas ] profunda paupertas 61757677 88* AMst PEL ${ }^{\text {var, }}$, lac.

64

eorum ] ipsorum 51, illorum 58 AMst, lac. 64

abundauit $5154^{c} 5861$ ] abundabit $54^{*}$, lac. 64, abundau[2] $89^{*}$

in ] lac. 64, ut in 89, om. AMstvar

diuitias $\left.\mathrm{AMst}^{\mathrm{R}}\right]$ diuitiis $5^{1} 54757677\left\langle 89^{*}\right\rangle \mathrm{AMst}^{\mathrm{ed}}$, lac. 64

simplicitatis eorum 61 ] simplicitas ipsorum $5^{1} 5^{8} \mathrm{PEL}^{\mathrm{B}}$, lac. 64

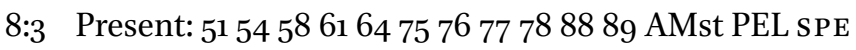

quia ] quoniam $6177 \mathrm{PEL}^{\mathrm{B}}$, lac. 64, quod $\mathrm{AMst}^{\mathrm{R}}$

secundum uirtutem ] pro uiribus 6177 AMst $\mathrm{PEL}^{\mathrm{B}}$, lac. 64, secundum uir[es] $88^{*}$

testimonium AMst ${ }^{\mathrm{var}}$ ] testimonii 58 , lac. 64, testimonio AMsted $\mathrm{PEL}^{\mathrm{B}}$

illis ] lac. 64, om. $75767788 * 89$

reddo ] lac. 64, perhibeo $88^{*}$, sum AMst, sumus $\mathrm{PEL}^{\mathrm{B}}$; + uel testor reddo 77 et ] quod $88^{\mathrm{C}} \mathrm{PEL}^{\mathrm{var}} \mathrm{SPE}^{\mathrm{var}}$ 
supra $A M s t^{R}$ ] ultra $61 \mathrm{AMst}^{\text {ed }} \mathrm{PEL}^{\mathrm{B}}$, per uel ultra 77 , super $89 \mathrm{PEL}^{\mathrm{var}}$ SPEvar

uirtutem ] uires $616488^{*}$ AMst $\mathrm{PEL}^{\mathrm{B}}$, uires uel uiribus 77 , uirtute 89 uoluntarii fuerunt ] sua sponte 757689 , uoluntarie fuerunt PEL var

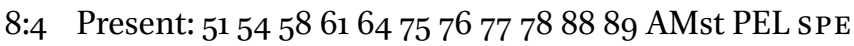
cum $\langle 64\rangle$ ] et cum 61, tum AMst ${ }^{\text {var }}$ multa exhortatione $547888^{c}$ ] precibus multis 61 , multis precibus $6488^{*}$ AMst, multa prece 7576 , multa precatione uel adhortatione 77 , multa obsecratione PEL ${ }^{\text {var }}$, multa exhortatio SPE ${ }^{\text {var }}$

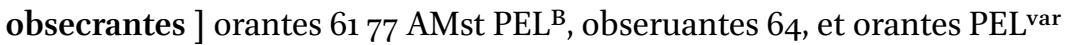
nos ] nostram $6177 \mathrm{AMst}^{\text {ed }} \mathrm{PEL}^{\mathrm{B}}$, suscipi in $\mathrm{AMst}^{\mathrm{R}}$ gratiam et communicationem 61 ] gratiam et communionem 64 (88*) AMst ministerii ] ministrationis 64, misterii $\mathrm{PEL}^{\mathrm{B}}$ quod ] quae 64, quam $77 \mathrm{PEL}^{\mathrm{B}}$, [4-6] qu[1] 88* fit $\mathrm{AMst}^{\mathrm{R}}$ ] habemus $77 \mathrm{PEL}^{\mathrm{B}}$, sit 88 , est $\mathrm{AMst}^{\text {ed }}$ in sanctos

8:5 Present: $51545^{8} 61647576777888$ 89 AMst PEL sPE et non ] sed non $\mathrm{AMst}{ }^{\mathrm{AD}}$ sicut ] quomodo $6488^{*}$, ut $\mathrm{AMst}^{\mathrm{R}}$ sperauimus ] speramus 7576 , sperabamus $\mathrm{AMst}^{\mathrm{R}} \mathrm{PEL}^{\mathrm{B}}$ sed ] quin immo $\mathrm{AMst}^{\mathrm{R}}$, sed et PEL var semet ipsos ] se ipsos $6488 * 89$, semet ipsi 7576 dederunt primum ] tradiderunt primum 64, [1-3]diderunt primum $88^{*}$, primum dederunt AMst domino ] deo $616478 \mathrm{AMst}^{\mathrm{PEL}} \mathrm{B}^{\mathrm{B}}$ deinde nobis ] et nobis $6488^{*}$, deinde et nobis 77 per uoluntatem dei 54 ] pro uoluntate dei AMstrar, per uirtutem dei SPEvar

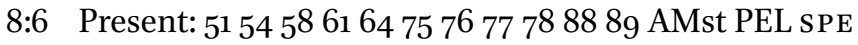

ita ut rogaremus ] ad deprecandum $6488^{*}$, ita ut exhortemur 89 , ita ut rogemus AMst ${ }^{\text {var; }}+$ nos 77

titum ut 58 ] titum quod $\mathrm{AMst}^{\mathrm{R}}$

quemadmodum ] sicut $61 \mathrm{PEL}^{\mathrm{B}}$, quomodo $6488^{*}$, sicuti 77 coepit ita 51545861 ] coepit sic $6488^{*}$

et ] ut 78, om. 89 AMst PEL ${ }^{\text {** }}$

perficiat ] consummet $64 \mathrm{AMst}^{\mathrm{ed}}$, lac. $88^{*}$, consumet AMst ${ }^{\mathrm{var}}$, perficiet $\mathrm{PEL}^{\mathrm{A} *} \mathrm{SPE}^{\mathrm{var}}$

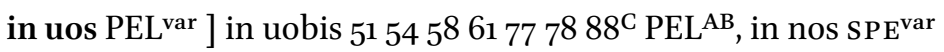


etiam (75) (76) ] om. 58 89 PEL var

gratiam istam ] gratiam ipsam $61^{*}$

8:7 Present: 5154586164757677788889 AMst PEL SPE

sed ] et 89 , uos autem AMst

sicut ] quomodo $6488^{*}$, quemadmodum AMst; + semper $64757688^{*} 89$

in omnibus abundatis $\left.5154^{c}{ }_{5} 861 \mathrm{AMs} t^{e d}\right]$ abundatis in omnibus 64, abun-

datis [...] $88^{*}$, in omnia abundatis 89 , in omnibus abundetis AMst ${ }^{\text {var }}$ $P E L^{\text {var }}$, in omnibus abundantes $P E L^{\text {var }}$; + in PEL ${ }^{\mathrm{B}}$

fide ] + spe 58

et sermone et scientia ] et uerbo et scientia (64) $7788^{*}$

et omni sollicitudine $5867(89)$ ] et omni studio 64 , et omni [...] 88*

et $\mathrm{AMst}^{\mathrm{R}}$ ] insuper et $5^{1} 545^{861}{ }_{78} \mathrm{PEL}^{\mathrm{B}}$, et insuper $75767789 \mathrm{AMst}^{\mathrm{ed}} \mathrm{PEL}^{\mathrm{var}}$, lac. $88^{*}$, et super AMst ${ }^{\text {var }}$

caritate uestra in nos $\left(8^{\mathrm{C}}\right)$ ] eam quae ex nobis in uobis est caritatem 64 , uestra in nos caritate 757677 (89), [...] caritate $88^{*}$, illa quae ex nobis in uobis est caritate AMst ${ }^{\text {ed }}$, illam quae ex nobis et uobis est caritatem $\mathrm{AMst}^{\mathrm{var}}$, illa quae ex nobis est caritate AMstrar, in caritate uestra in nos $\mathrm{PEL}^{\mathrm{B}}$; + facite $\mathrm{AMst}^{\mathrm{R}}$

ut et in ] ut $54^{*}$, et ut in 7576 , ut in $88^{*}$ AMstvar, et in 89

hac gratia 88 ] hac quoque gratia $\mathrm{AMst}^{\mathrm{R}}$

abundetis $5154^{c} 5861$ ] abundatis $88^{*}$; + magis AMsted

8:8 Present: 51545861647576777888 89 AMst PEL sPE

non quasi imperans ] non ut imperans 61 , non secundum imperium 64 AMst, non quasi inspirans $\mathrm{PEL}^{\mathrm{B}}$, non [...] 88*

dico sed ] lac. 88*

per PEL var ] propter $58616475767789 \mathrm{AMst}^{\mathrm{P}} \mathrm{LL}^{\mathrm{AB}}$, pro PEL ${ }^{\mathrm{var}}$, lac. 88*

aliorum ] om. $\mathrm{SPE}^{\mathrm{var}}$, lac. $88^{*}$

sollicitudinem 5861 ] studium 64 , sollicitudine PEL ${ }^{\text {var }}$, lac. 88*

etiam ] et 647576 AMst, ut 89 , lac. $88^{*}$

uestrae caritatis $51545^{8} 7788^{C}$ ] lac. $88^{*}$

ingenitum bonum conprobans ] ingenium bonum conprobans 51545861 $7888^{\mathrm{C}} \mathrm{PEL}^{\mathrm{var}} \mathrm{SPE}$, carissimum probans 64 , ingenium probans $757^{6} 89$, bonum conprobans $77 \mathrm{AMst}^{\mathrm{var}}$, lac. 88*, bonum probans AMsted, bonum animum probans $\mathrm{AMst}^{\mathrm{R}}$, ingenium conprobans $\mathrm{PEL}^{\mathrm{AB}}$

8:9 Present: 51545861647576777888 89 AMst PEL CAr SPE

scitis ] nostis AMst, scimus SPEvar

enim ] om. PEL var 
gratiam domini ] gratia domini 6489

nostri iesu christi AMst ${ }^{\mathrm{var}}$ ] iesu christi $89 \mathrm{AMst}^{\mathrm{ed}}$

quoniam PEL var ] quia $61757677 \mathrm{AMst}^{\mathrm{ed}} \mathrm{PEL}^{\mathrm{AB}}$, quem 89, qui $\mathrm{AMst}^{\mathrm{var}}$, abs.

CAr

propter uos AMst ${ }^{\text {var }}$ ] propter nos $5158^{*} \mathrm{AMst}^{\mathrm{ed}}$, abs. CAr

egenus ] pauper $616475767788^{*}$ AMst PEL $^{\mathrm{B}}$, abs. CAr

factus est cum $\left(5^{*}\right)$ ] fuit ut 89 , abs. CAr

esset diues ut 54 PEL $^{\text {var }}$ ] diues esset ut 61647576778889 AMst $^{\text {ed }}$ PEL $^{\mathrm{AB}}$, ditaremini ut $\mathrm{AMst}^{\mathrm{R}}$, abs. CAr

illius inopia $S P E^{\mathrm{var}}$ ] illius paupertatem 64 , in illius paupertate uel inopia (77), illius paupertate $88^{*}$, illius egentia $\left(89^{*}\right)^{\mathrm{C} *}$, inopia illius SPE ${ }^{\mathrm{ed}}$, abs. CAr

uos diuites essetis ] uos ditaremini $6488^{*}$, uos ditaremini uel diuites essetis 77 , nos diuites essemus 89 , uos honestaremini $\mathrm{PEL}^{\mathrm{B}}$, diuites essetis SPE ${ }^{\mathrm{var}}$, abs. CAr

8:10 Present: 51545861647576777888 89 AMst PEL sPE

et consilium in hoc do ] et consilium in hoc de 89 *

hoc enim uobis $(78)$ ] hoc enim $\mathrm{PEL}^{\mathrm{B}}$

utile est ] prodest $6488^{*}$

qui AMstrar ] quia $647778 \mathrm{AMst}^{\text {ed }} \mathrm{PEL}^{\text {var }}$, om. 89, quod $\mathrm{PEL}^{\mathrm{B}}$, quoniam PELvar

non solum ] non tantum 61 AMst PEL ${ }^{\mathrm{B}}$, non tantum uel solum 77

facere sed et uelle ] autem uellem 89

coepistis 5154586188 ] + laborare 7576

ab anno priore ] ab anno praeterito $61 P E L^{B}$; + uel praeterito 77

8:11 Present: $51545^{8} 61647576777888$ 89 AMst PEL SPE

nunc uero ] nunc ergo 61 AMst, nunc autem $647788^{*} \mathrm{PEL}^{\mathrm{B}}$, nunc uere $\mathrm{PEL}^{\mathrm{var}}$ et ] etiam $\mathrm{AMst}^{\mathrm{R}}$, om. AMst ${ }^{\mathrm{var}}$

facto (78) $\mathrm{AMst}^{\mathrm{R}}$ ] facio 7576 , facere $\mathrm{AMst}^{\mathrm{ed}}$

perficite ut quemadmodum ] consummate ut quemadmodum AMst

promptus $5488 S P E$ ] prompta $6177 \mathrm{AMst}^{\mathrm{PEL}}{ }^{\mathrm{B}}$, animus 7576

est ] fuit $\mathrm{AMst}^{\mathrm{R}}$

animus ] animis 54, uoluntas 61 AMst, om. 757677 PEL ${ }^{\mathrm{B}}$; + in uobis 6177 AMst PEL ${ }^{B}$

uoluntatis $54 * 89$ ] benefaciendi 61 , uoluntas 77 , uolendi $78 \mathrm{AMst}^{\mathrm{R}}$, uelle AMsted, uoluntas faciendi PEL ${ }^{\mathrm{B}} ;+[\ldots] 78^{\dagger}$

$\dagger{ }_{2}$ Cor. 8:11. There is a blank space left at this point in both Latin and Greek columns of $\mathrm{VL} 78$. 
ita sit et perficiendi ] ita sit ad perficiendum $51 ;+$ uel consummandi uel finiendi 77

ex eo quod habetis 54 ] ex eo quod quisque habet 64 , ex abundantia 7576 89 , ex habentia uel ex eo quod habetis 77 , ex eo quod [4-6] habetis $88^{*}$

8:12 Present: 51545861647576777888 89 AMst PEL tes SPE

si enim ] nam si 757689 , si TES

uoluntas prompta est $5467(78)\left(88^{\mathrm{C}}\right) \mathrm{SPE}^{\mathrm{var}}$ ] prompta uoluntas est $5^{1}$, promptus est animus 6489 , promptus animus est 7576 , uoluntas prompta uel proposita est 77 , promptus est $88^{*}$, uoluntas est $\mathrm{PEL}^{\mathrm{B}}$, uoluntas prompta PEL ${ }^{\mathrm{var}} S P E^{\text {ed }}$

secundum ] lac. $88^{*}$

id quod habet 54 ] quod habet 757689 , lac. $88^{*}$, facultatem AMsted, uoluntatem AMstvar, quodcumque habuerit TES $S^{\text {ed }}$, quod quisque habuerit $\mathrm{TE} \mathrm{s}^{\mathrm{var}}$, quod unusquisque habuerit TES ${ }^{\mathrm{var}}$, quod habuerit TES $\mathrm{S}^{\mathrm{var}}$

accepta 54 ] acceptabilis 64 AMst, acceptus 7576 89, bene acceptum 77, lac. $88^{*}$, acceptabile $\mathrm{TES}^{\mathrm{ed}}$, neque acceptabilis $\mathrm{TES}^{\mathrm{var}}$, iucundumquodque plus acceptabile TES $\mathrm{S}^{\mathrm{var}}$

est non ] est [...] 64, lac. $88^{*}$

secundum quod $\left.\left(64^{*}\right)\right]$ secundum id quod $545888 \mathrm{PEL}^{\mathrm{B}}$

non habet $\left.54 \mathrm{TES}^{\mathrm{var}}\right][\ldots]$ habet $88^{*}$, non habuerit $\mathrm{TES}^{\mathrm{ed}}$

8:13 Present: $51545^{8} 61757677788889$ AMst PEL tes SPE

non ] quis non 77 , neque TES $S^{\text {ed }}$, ut TES ${ }^{\text {var }}$

enim ] om. 61 TES; + dico 58

ut aliis ] ut alii AMst ${ }^{\mathrm{var}}$, sit aliis TES ${ }^{\mathrm{var}}$, aliis TES $\mathrm{S}^{\mathrm{var}}$

sit remissio ] refectio sit $6177^{*}$, refectio $77^{\mathrm{C}} \mathrm{PEL}^{\mathrm{B}}$, sit refectio $88^{*}$, remissio

89 , refrigerium sit $\mathrm{AMst}^{\mathrm{ed}}$, relaxatio sit $\mathrm{AMst}^{\mathrm{R}}$, refrigerium $\mathrm{TES}^{\mathrm{ed}}$, sit refrigerium TES $S^{\text {var }}$

uobis autem TES var $^{\text {] uobis }} 757688^{*} \mathrm{TES}^{\mathrm{ed}}$

tribulatio ] angustia $6188^{*} \mathrm{AMst}^{\mathrm{ed}} \mathrm{PEL}^{\mathrm{B}}$, angustias AMst ${ }^{\mathrm{var}}$, praessura TEs; + sit 89 TES $^{\text {ed }}$

sed ex ] sed pro $88^{*}$, sed et ex SPE ${ }^{\mathrm{var}}$, abs. TES

aequalitate $587888 \mathrm{AMst}^{\mathrm{R}}$ ] qualitate $5^{1} 54$, aequitate $757^{6 \mathrm{AMst}^{\mathrm{ed}}} P E L^{B}$, abs. TES

8:14 Present: $51545^{8} 61757677788889$ AMst PEL TES SPE

in praesenti tempore $545^{8} 7888^{C} 89$ ] in hoc tempore praesenti 51 , in hoc praesenti tempore 61 , in hoc tempore $757^{6} 77^{\mathrm{C}} 88^{*} \mathrm{AMst}_{\mathrm{PEL}}^{\mathrm{B}}$, in hoc nunc tempore $77^{*}$, abs. TES 
uestra ] ut uestra $586188 * 89$ AMst, nostra autem TES ${ }^{\text {var }}$ abundantia 51545861$]+$ inopiam 58

illorum $\mathrm{AMst}^{\mathrm{R}}$ ] ad illorum $7576 \mathrm{AMst}^{\mathrm{ed}}$, sit ad illorum $7788^{*}$, sit illorum $88^{\mathrm{C}}$, abundet in aliorum 89 , fiat ad illorum AMst ${ }^{\mathrm{var}}$

inopiam ] om. 58 , inopia $7576 \mathrm{PEL}^{\text {var }}$ TES $^{\mathrm{var}}$

suppleat ] om. $75767788^{*} 89$ AMst, impleat PEL var

ut et $S P E^{\text {var }}$ ] et 61 AMst PEL var TES ${ }^{\text {var }} S P E^{\text {ed }}$, et ut PEL var TES ${ }^{\text {var }}$, ut PEL var $S P E^{\text {var }}$

illorum abundantia 5154586188$]$ + sit ad $6175767789,+$ lac. $88^{*},+$ fiat ad $\mathrm{AMst}^{\mathrm{ed}},+$ sit PEL ${ }^{\mathrm{ed}},+\operatorname{sic} \mathrm{PEL}^{\mathrm{B}}$

uestrae inopiae 58 ] inopiae uestrae 51 , uestra inopiae 54 , uestram inopiam 75767789 AMst $^{\text {ed }}$, uestr[1-2] inopi[1-2] 88*, uestram suppleat inopiam $\mathrm{AMst}^{\mathrm{R}}$, uestra inopia TES $\mathrm{S}^{\mathrm{var}}$

sit supplementum ] supplementum $61 \mathrm{PEL}^{\mathrm{B}}$, om. $75767788^{*} 89$ AMst, est supplementum TE $\mathrm{S}^{\mathrm{var}}$

ut fiat ] et sit TES $S^{\text {ed }}$, et sit TES ${ }^{\text {var }}$, ut et sit TES ${ }^{\text {var }}$; + in omnibus 61

aequalitas $51545888 \mathrm{AMst}^{\mathrm{var}}$ ] aequitas $77 \mathrm{AMst}^{\mathrm{ed}}$

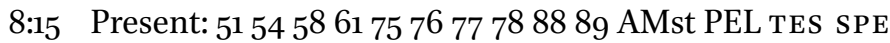

sicut scriptum est ] + in exodo 61

qui multum Tes ${ }^{\text {var }}$ ] qui habuit multum 51, qui multum habuit $54^{\mathrm{C}} 5^{8} 61$ AMst Tes ${ }^{\text {ed }}$, qui multum habet 88

non abundauit 5154586188 ] non abundabit $P E L^{v a r} \mathrm{TES}^{\mathrm{var}} \mathrm{SPE}^{\mathrm{var}}$

et ] ut AMstrar, om. TES ${ }^{\text {var }}$

qui modicum non

minorauit ] minuit $77,[6-8] 88^{*}$, minorabit $\mathrm{PEL}^{\mathrm{var}} \mathrm{SPE}^{\mathrm{var}}$, indiguit TES

8:16 Present: $51545^{8} 61757677788889$ AMst PEL SPE

gratias 88 ] gratia 757677

autem deo ] agimus deo PEL var

qui dedit ] danti 77

eandem ] [3] dem studium 88*, hanc ipsam AMst, eam PEL var

sollicitudinem 5861 ] om. $88^{*}$, sollicitudium $88^{\mathrm{C}}$

pro uobis ] om. $\mathrm{PEL}^{\mathrm{B}}$

in corde titi 58 ] in cor titi AMst ${ }^{\mathrm{var}}$

8:17 Present: 51545861757677788889 AMst PEL SPE

quoniam ] qui $S P E^{\text {var }}$

exhortationem quidem $5478 \quad 88^{C}$ AMst $^{\mathrm{R}}$ ] consolationem quidem 61 $\mathrm{AMst}^{\text {ed }} \mathrm{PEL}^{\mathrm{B}}$, quidem exhortationem 7576 , quidem consolationem 77 , [6-10] onem quidem $88^{*}$ 
suscepit ] accipit 61 AMst ${ }^{\mathrm{var}}$, accepit $77 \mathrm{AMst}^{\mathrm{ed}} \mathrm{PEL}^{\mathrm{B}} \mathrm{SPE}^{\mathrm{var}}$, suscipit SP Evar; + a me $5^{1}$

sed cum sollicitior esset 51 ] sed dum sollicitior esset $545^{8}$, cum autem sit sollicitior 61, sollicitior autem cum esset $757_{6}^{6} 77$, lac. $88^{*}$, cum sit autem sollicitior AMst PEL ${ }^{\mathrm{B}}$

sua uoluntate ] uoluntarius $757677 \mathrm{AMst}^{\mathrm{PEL}} \mathrm{L}^{\mathrm{B}}$, lac. 88*

profectus est ad uos ] [...] ad uos $88^{*}$

8:18 Present: 51545861757677788889 AMst PEL SPE

misimus $6177^{*}$ ] missus PEL $L^{\text {var }}$

etiam $\mathrm{AMst}^{\mathrm{R}}$ ] autem $75767789 \mathrm{AMst}^{\mathrm{ed}} \mathrm{PEL}^{\mathrm{B}}$ SPE, om. 88*

cum illo ] [2] um eo $88^{*}$, om. $\mathrm{PEL}^{\mathrm{B}}$

fratrem ] fratrem nostrum $546189 \mathrm{AMst}^{\mathrm{PEL}} \mathrm{L}^{\mathrm{B}}$, fratrem nostrum lucam $5^{8}$, fratres PEL ${ }^{\text {var }}$

cuius laus est in euangelio $5458 \mathrm{AMst}^{\mathrm{var}}$ ] cuius laus in euangelio est 6188 AMst $^{\text {ed }} P E L^{B}$, cuius laus in euangelio 75767789

per omnes ecclesias ${ }_{51} 61$ ] per omnem ecclesiam $\mathrm{SPE}^{\mathrm{var}}$

8:19 Present: 51545861757677788889 AMst PEL SPE

non solum autem ] om. PELA

sed ] uerum $5177 \mathrm{PEL}^{\mathrm{B}}$, hoc uerum 61

et ] etiam $5^{1} 6177$ AMst PEL ${ }^{\mathrm{B}}$, om. $7576 \mathrm{PEL}^{\text {var }}$

ordinatus ab ecclesiis $5154^{c}$ ] ordinatus est ab ecclesiis 58 6r $77^{*}\left(88^{*}\right)$ AMst $^{\text {ed }}$ PEL $^{\mathrm{B}}$

comes peregrinationis nostrae 545888 ] comes nostrae peregrinationis 51 , comes peregrinationis meae 61 , comes noster 75767789

in ] cum $6175767788^{*} 89 \mathrm{PEL}^{\mathrm{B}}$

hac gratia 88 ] hanc gratiam $54 \mathrm{AMst}^{\mathrm{R}}$, gratia hac 77 , hac gloria $\mathrm{AMst}^{\mathrm{AD} *}$, hac gratiae $S P E^{\text {var }}$

quae ministratur $5^{1} 545^{8} 6188$ ] ministrata uel quae ministratur 77 , quae ministrat $\mathrm{PEL}^{\mathrm{B}}$, qui ministratur $\mathrm{SP} \mathrm{E}^{\mathrm{var}}$, ministratur $\mathrm{SPE}^{\mathrm{var}}$

a nobis ] om. 89, nobis PEL var

ad $\left.\left(88^{*}\right) \mathrm{AMst}^{\mathrm{R}}\right]$ per AMsted; + eandem $5^{1}$

domini ] deum PEL ${ }^{\mathrm{B}}$

gloriam et ] gratiam et $61^{*}$

destinatam uoluntatem nostram $54^{*} 61$ ] destinatam nostram uolun 75 76 , destinatam nostram uoluntatem 77 , lac. $88^{*}$, sollicitudinem nostram $\mathrm{AMst}^{\mathrm{ed}}$, promptitudinem uestram $\mathrm{AMst}^{\mathrm{R}}$, distinctam uoluntatem nostram $\mathrm{SPE}^{\mathrm{var}}$ 
8:20 Present: $51545^{8} 61757677788889$ AMst PEL SPE

deuitantes hoc 61 ] + quod PELvar

ne quis nos uituperet $\left(54^{*}\right) \mathrm{AMst}^{\mathrm{var}}$ ] ne quis nos reprehendat $516188^{*} \mathrm{PEL}^{\mathrm{B}}$, ne quis uituperet nos $\mathrm{AMst}^{\mathrm{ed}}$, ne quis uos reprehendat $\mathrm{PEL}^{\mathrm{var}}$

in hac plenitudine ] in plenitudine hac 757677

quae 5154586188 ] om. 77 , qui SPEvar

ministratur ] ministrata 77 , ministrat PEL var

a nobis ] nobis $S \mathrm{E}^{\mathrm{var}}$

\pm in gloriam dei 51 , in gloria domini 54 , in gloriam domini 58 , in gloria dei $88^{\mathrm{C}}$, in domini gloriam $\mathrm{AMst}^{\mathrm{R}}$

8:21 Present: 515458617576777888 89 AMst PEL sPM SPE

prouidemus enim ] prouidimus enim PEL ${ }^{\text {var }}$, prouidentes enim SPM ${ }^{\text {var }}$ bona ] bonam PELvar

non solum ] non tantum PEL var SPM

coram deo ] coram domino 757677

sed etiam $\mathrm{AMst}^{\mathrm{var}}$ ] sed et $587789 \mathrm{SPE}^{\mathrm{var}}$, uerum etiam $61 \mathrm{AMst}^{\mathrm{ed}} \mathrm{PEL}^{\mathrm{B}}$, sed

7576

coram hominibus ] coram omnibus $54^{\mathrm{C}} \mathrm{SPE}^{\mathrm{var}}$, coram omnibus hominibus $5^{8}$

8:22 Present: 515458617576777888 89 AMst PEL CAr

misimus autem 61 ] misimus etiam 51 , misimus $P L^{B}$

cum illis ] cum eis $88^{*}$, om. $\mathrm{PEL}^{\mathrm{B}}$, cum illo CAr

et ] om. $61^{*} 75767788 \mathrm{CAr}$, ad AMstvar

fratrem nostrum ] + apollo $58,+$ cum illis $\mathrm{PEL}^{\mathrm{B}}$

quem probauimus (89) ] quem probabimus $7576 \mathrm{CAr}^{\mathrm{var}}$

in multis saepe 515458617778 ] saepe in multis 88 , saepe CAr

sollicitum 5861 ] sollicitantem uel sollicitum 77 , studiosum $88^{*}$, abs. CAr

esse nunc autem multo 54] esse nunc uero multo $7576 \mathrm{AMst}^{\mathrm{PEL}} \mathrm{L}^{\mathrm{B}}$, abs. CAr sollicitiorem 5861 ] lac. $88^{*} \mathrm{CAr}$; + multa 61 AMst

confidentia $88^{C}$ ] confidentia[1-2] $88^{*}$, confidentiam 89, fiducia AMst, lac.

CAr; + mihi $58,+$ uestri AMst PEL ${ }^{\mathrm{B}}$

multa ] om. 6189 AMst, quae 77 , abs. CAr

in uos ] in uobis $5^{1} 7789 \mathrm{PEL}^{\mathrm{var}}$, in nobis 75 76, om. AMst $\mathrm{PEL}^{\mathrm{B}}$, lac. CAr; + habuit 89

8:23 Present: 515458617576777888 89 AMst PEL

siue pro tito 58 ] siue per titum AMst

qui est socius meus ] socius meus $75767788^{*} 89$ 
et ] om. $89 \mathrm{AMst}^{\mathrm{AD}}$

in uos adiutor $\mathrm{PEL}^{\text {var }}$ ] adiutor in uobis $617576 \mathrm{AMst}^{\mathrm{PEL}} \mathrm{L}^{\mathrm{B}}$, in uobis adiutor $7788^{\mathrm{C}}$ PELA $^{\mathrm{A}}$, in uos cooperatur $88^{*}$

siue fratres nostri apostoli ] siue pro fratribus nostris apostolis 51 , siue pro his qui sunt fratres nostri apostoli 61, siue per fratres nostros apostolos AMst

ecclesiarum gloriae christi 5154586188 ] ecclesiarum gloria christi $757^{6} 77$ 7889

8:24 Present: 515458617576777888 89 AMst PEL

ostensionem $\mathrm{AMst}^{\mathrm{R}}$ ] et ostensionem $88^{*}$, ostentationem $\mathrm{AMst}^{\mathrm{ed}}$ ergo ] om. $88 * 89$ quae est $51545^{8}$ ] om. $617576777^{8} 88^{*}$ AMst, quae sit 89 caritatis ] caritas $\mathrm{PEL}^{\mathrm{B}}$ uestrae et nostrae $51545^{8}(75)(76) 7788$ ] nostrae et uestrae $61^{*}$ gloriae pro uobis $515488^{c}$ ] pro uobis gloriae $5888^{*}$, gloriae uel exultationis pro uobis 77 , pro uobis exultationis $\mathrm{AMst}^{\mathrm{ed}}$, pro uobis exhortationis $\mathrm{AMst}^{\mathrm{AD} *}$, exultationis pro uobis $\mathrm{PEL}^{\mathrm{B}}$

in ] demonstrate in AMst

illos PEL var $]$ illis 54 PEL $^{\text {var, ipsos }} 757^{6} 77,[1-2] \mathrm{s}[1-2] 88^{*}$, illo 89 PEL $^{\mathrm{A}}$, eos $\mathrm{AMst}^{\mathrm{R}}$, ipsis AMst ${ }^{\text {ed }} \mathrm{PEL}^{\mathrm{B}}$, illos PEL var

ostendite 54 ] ostendentes $75767788^{*} 89$, om. AMst in faciem AMst ${ }^{\mathrm{var}} \mathrm{PEL}^{\mathrm{var}}$ ] in facie $515889 \mathrm{AMst}^{\mathrm{ed}} \mathrm{PEL}^{\mathrm{AB}}$ ecclesiarum $\left.5_{1} 6188^{*}\right]+$ dei $5158,+$ domini PEL $^{\mathrm{B}}$

9:1 Present: 51545861757677788889 AMst PEL SPE nam de ] iam de 58 , de quidem enim 77 , nam $\mathrm{SPE}^{\mathrm{var}}$ ministerio ] misterio 58 quod fit in sanctos ] quod fit in sanctis $88^{\mathrm{C}}$ ex abundanti $51545^{8} \mathrm{SPE}^{\mathrm{var}}$ ] ex abundantia $6189 \mathrm{PEL}^{\text {var }} \mathrm{SPE}^{\mathrm{ed}}$, abundanti[1] $77^{*}$, abundantius $77^{\mathrm{C}}$, superuacuum AMst ${ }^{\text {ed }}$, superfluum $\mathrm{AMst}^{\mathrm{R}}$ est mihi scribere uobis $5^{8}$ ] mihi est scribere uobis $5^{1} 777^{*}$

9:2 Present: 51545861757677788889 AMst PEL SPE scio enim promptum $54\langle 76\rangle 88 S P E]$ scio enim $6{ }_{17} \mathrm{PEL}^{\mathrm{B}}$, scio enim promptam AMst; + [...] 88* animum uestrum ] uoluntatem uestram $6177 \mathrm{AMst}^{\mathrm{PEL}} \mathrm{L}^{\mathrm{B}}$ pro $\mathrm{AMst}^{\mathrm{R}}$ ] de $757689 \mathrm{AMst}^{\mathrm{ed}}$, om. $77 \mathrm{PEL}^{\mathrm{B}}$, lac. $88^{*}$ quo ] qua 61 AMst $\mathrm{PEL}^{\mathrm{B}}$, quam 77 , lac. $88^{*}$

de uobis glorior $\mathrm{AMst}^{\mathrm{R}}$ ] glorior de uobis 75 76, lac. $88^{*}$, glorior in uobis 89 , pro uobis glorior AMst ${ }^{\text {ed }}$ 
apud macedonas $\left.545^{8} 7576 A M t^{e d}\langle\mathrm{SPE}\rangle\right]$ apud macedones $5^{1} 6188^{*}$ $\mathrm{AMst}^{\mathrm{var}} \mathrm{PEL}^{\mathrm{B}}$, apud macedoniam 89 quoniam ] quia 75767789 ; + et $51545^{8}$ achaia parata est ] achaia praeparata est $75767789 \mathrm{PEL}^{\text {var }}$ ab anno ] et anno AMst ${ }^{\mathrm{var}}$, om. AMst ${ }^{\mathrm{var}}$ praeterito $51545^{8} 6188^{C}$ ] priore $75767889 \mathrm{AMst}^{\mathrm{ed}}$, praeterito uel priore 77 , $\operatorname{pr}[16-20] 88^{*}$, primo AMst ${ }^{\mathrm{var}}$, om. AMst ${ }^{\mathrm{var}}$ et ] lac. $88^{*}$, om. AMst $^{\text {var }} \mathrm{PEL}^{\mathrm{var}}$, ut PEL ${ }^{\mathrm{var}}$

uestra aemulatio $51545^{8} 7888^{c}$ ] aemulatio uestra 6189 , quae ex uobis est aemulatio 7576 , quae ex uobis aemulatio 77 , lac. $88^{*}$, uestra aemulatione $\mathrm{AMst}^{\mathrm{var}}$, uestra enim aemulatio PEL ${ }^{\text {var }}$, uestrae aemulatio PEL ${ }^{\text {var }}$ prouocauit ] prouocabit 7576 , lac. $88^{*}$, prouocat AMst ${ }^{\mathrm{var}}$ plurimos ] plures $61757677 \mathrm{AMst}$, multos 78 , lac. 88*

9:3 Present: 51545861757677788889 AMst PEL SPE misi ] misimus $545^{8} 6177^{88^{\mathrm{C}}} \mathrm{AMst}^{\mathrm{var}} \mathrm{PEL}^{\mathrm{B}} \mathrm{SPE}^{\mathrm{var}}$, lac. 88* autem ] lac. $88^{*} ;+$ et $\mathrm{AMst}^{\mathrm{ed}}$, + hos $\mathrm{AMst}^{\mathrm{R}}$ fratres ] lac. 88*, fratrem AMstrar ut ne ] ut $61 \mathrm{PEL}^{\mathrm{B}}$, uti non 75 76, lac. $88^{*}$, uti ne 89 , ne AMst \pm exultatio nostra $6177 \mathrm{PEL}^{\mathrm{B}}$, gloria nostra AMst quod ] quam $61 \mathrm{PEL}^{\mathrm{B}}$, om. 77 , lac. 88*, id quod 89, qua AMst; + uos AMst ${ }^{\text {ed }}$ gloriamur ] habemus 61, gloriamus 7576 lac. $88^{*}$, om. $77 \mathrm{PEL}^{\mathrm{B}}$, praeferimus AMst

de uobis ] om. 77 AMst, lac. 88*; + habemus PEL ${ }^{\mathrm{B}}$ euacuetur ] non euacuetur $61 \mathrm{PEL}^{\mathrm{B}}$, exinaniatur 7576 89, lac. $88^{*}$, inanis fiat AMst in hac parte ] in parte hac 77 , lac. $88^{*}$, in hanc partem $\mathrm{AMst}^{\mathrm{var}}$ ut ] et 54 , et ut 58 , lac. $88^{*}$ quemadmodum ] lac. $88^{*}$ dixi ] dixeram 757689 ; + uobis PEL var parati sitis $\mathrm{AMst}^{\mathrm{R}}$ ] praeparati sitis $\mathrm{AMst}^{\mathrm{ed}}$

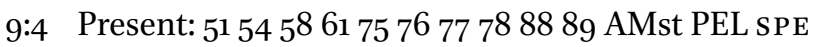
ne ] ut 58 , et ne 7576 , ne si 89 cum uenerint ] cum uenerunt 5461 mecum macedones 61757689 ] macedones mecum AMstrar, mecum in macedones PEL var et inuenerint ] inuenerint 61 uos ] nos $78 \mathrm{PEL}^{\mathrm{B}}$ inparatos 587778$]+$ et 7576 
erubescamus ] confundamur 757689 , lac. 88*

$\left.\operatorname{nos}\left(5^{*}\right) \mathrm{AMst}^{\mathrm{R}}\right]$ om. AMsted; + in hac parte ${ }_{54}{ }^{\mathrm{C}} 5861 \mathrm{PEL}^{\mathrm{B}}$

ut non ] ne 7576 , om. $89 \mathrm{AMst}^{\mathrm{A}}$, ut nos AMsted

dicamus $\mathrm{SPE}^{\mathrm{var}}$ ] abdicamus 58 , dicam $6175767788 \mathrm{AMst}^{\mathrm{ed}} \mathrm{PEL}^{\mathrm{B}} \mathrm{SPE}^{\mathrm{ed}}$, om.

$89 \mathrm{AMst}^{\mathrm{A}}$; + omnes $61 \mathrm{AMst} \mathrm{PEL}^{\mathrm{B}}$

uos ] om. 89, nos AMst ${ }^{\mathrm{AD}}$, uobis PEL var

in hac substantia $54\left(78^{*}\right)$ ] om. $61 \mathrm{PEL}^{\mathrm{B}}$, in substantia hac 77 , in hac parte AMst; + gloriae 58

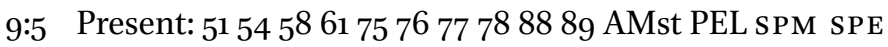
necessarium ergo $\mathrm{SPM}^{\mathrm{var}}$ ] necessarium autem $89 \mathrm{PEL}^{\mathrm{var}} \mathrm{SPM}^{\mathrm{ed}}$ existimaui ] exaestimaui $\mathrm{AMst}^{\mathrm{var}} P E L^{A}$, existimarent $\mathrm{AMst}^{\mathrm{var}}$, exaestimabit $S_{P M}{ }^{\text {var }}$

rogare fratres ut ] ut fratres $\mathrm{AMst}^{\mathrm{var}}$, fratres rogare ut $\mathrm{PEL}^{\mathrm{var}}$

praeueniant $51545^{8} 7788^{c} \mathrm{SPE}^{\mathrm{var}}$ ] praecedant 61 , pergant $757689 \mathrm{SPM}$, prae $[t] \mathrm{e}[r e]$ ant $88^{*}$, praecederent AMst, perueniant $\mathrm{PEL}^{\mathrm{B}}$, peruenirent $S P E^{\text {ed }}$, reuenirent $S \mathrm{PE}^{\mathrm{var}}$

ad uos $\left.P E L^{\text {ed }}\right]$ om. $P E L^{A}$, ad nos $P E L^{B}$

et ] ut $757688^{\mathrm{C}}$

praeparent $\left.5154587788 \mathrm{AMst}^{\mathrm{var}} \mathrm{SPE}^{\mathrm{var}}\right]$ praepararent $61 \mathrm{AMst}^{\mathrm{ed}} \mathrm{SPE}^{\mathrm{ed}}$, praeparant PEL ${ }^{\mathrm{var}}$; + ante $75767789 \mathrm{PEL}^{\mathrm{B}}$ SPM, + [...] 88*

repromissam PEL ${ }^{\text {var }}$ ] promissam 7576777889 AMst PEL $^{\mathrm{AB}} \mathrm{SPM}$ benedictionem 54 PEL $\left.^{\mathrm{var}}\right]$ hanc benedictionem $78\left\langle 88^{*}\right\rangle$ PEL $^{\mathrm{AB}}$, benedictionem hanc AMst; + uestram 7778 AMst PEL $^{\mathrm{B}}$

hanc ] om. 7778 AMst PEL ${ }^{\mathrm{A}}$, lac. $88^{*}$; + sed sic eam AMst ${ }^{\mathrm{R}}$

paratam esse 54 ] paratam $88^{*}$, esse paratam $\mathrm{AMst}{ }^{\mathrm{var}}$, om. $\mathrm{PEL}{ }^{\mathrm{var}}$; + sed AMst ${ }^{\text {ed }}$ SPM

sic ] om. AMst $^{\mathrm{R}}$

quasi benedictionem 5488 ] quo si benedictis SPM ${ }^{\text {var }}$

non quasi auaritiam ${ }_{51} 88$ ] non quasi circumuentionem AMst

9:6 Present: 5154586175767778848889 AMst PEL tes SPM SPE

hoc autem ] hoc autem dico $51545^{8} 61788^{\mathrm{C}} \mathrm{AMst}^{\mathrm{P}} \mathrm{LL}^{\mathrm{B}} \mathrm{SPM} \mathrm{SPE}^{\mathrm{var}}$, fratres hoc dico 84 , hoc est autem dico PEL var, om. TES

qui $\mathrm{AMst}^{\text {var }}$ ] quoniam qui 58 , quia qui $617884 \mathrm{AMst}^{\mathrm{ed}} \mathrm{SPM} \mathrm{SPE}^{\mathrm{var}}$, quia AMst $^{\mathrm{A}}$

parce seminat $\mathrm{SPM}^{\mathrm{var}}$ ] seminat parce $757677 \mathrm{SPM}^{\mathrm{ed}}$, parcit ${ }^{\dagger} 84$, seminat confidens 89, parce seminet AMst ${ }^{\mathrm{var}}$, sparget et seminat TES $\mathrm{S}^{\mathrm{var}}$

$\dagger \quad 2$ Cor. 9:6. Dold reconstructs the final word of VL 84 as parciter. 
parce et metet ] abs. 84, confidenter et metet 89, parce et metit $\mathrm{AMst}^{\mathrm{var}}$ $\mathrm{PEL}^{\mathrm{var}}$, sparget et metet TES ${ }^{\mathrm{var}}$, parcet metet TES ${ }^{\mathrm{var}}$, et metet SPM ${ }^{\mathrm{var}}$ et qui ] abs. 84, qui $89 \mathrm{PEL}^{\mathrm{B}} \mathrm{TES}^{\mathrm{var}} \mathrm{SPM}^{\mathrm{var}}$, qui autem AMst seminat in benedictionibus $88^{C} \mathrm{PEL}^{\mathrm{var}}$ ] seminat in benedictione 617576 $7788^{*}$ PEL $^{\mathrm{AB}}$ TES $^{\text {ed }}$ SPM, abs. 84, seminat in benedictionem $89 \mathrm{TES}^{\mathrm{var}}$, in benedictione seminat AMst

de $\mathrm{AMst}^{\mathrm{R}}$ ] in $6177 \mathrm{AMst}^{\mathrm{ed}}$, abs. 84, lac. 88*, ex 61 ${ }^{\text {alt }} \mathrm{PEL}^{\mathrm{B}}$, om. TES ${ }^{\mathrm{var}}$ benedictionibus PEL ${ }^{\text {var }}$ ] benedictione $6175767788^{*} 89$ AMst PEL $^{\mathrm{AB}}$ TES $^{\text {ed }}$ SPM, abs. 84, benedictionem TES ${ }^{\text {var }}$, om. TES $S^{\text {var }}$ et $\mathrm{AMst}^{\mathrm{var}}$ ] om. $61 \mathrm{AMst}^{\mathrm{ed}} \mathrm{PEL}^{\mathrm{B}} \mathrm{SPM}^{\mathrm{var}}, a b s .84$, in TES ${ }^{\mathrm{var}}$ metet ] abs. 84; + uitam aeternam 89 TES $^{\text {var }}$ SPM

9:7 Present: 515458617576777888 89 AMst PEL TES SPM SPE unusquisque ] + autem TES, + enim SPE ${ }^{\text {ed }}$ prout destinauit corde suo $\left.\mathrm{PEL}^{\text {var }}\right]$ prout destinauit in corde suo 5458 $\mathrm{PEL}^{\mathrm{AB}} \mathrm{SPE}^{\mathrm{var}}$, secundum propositum cordis $6177 \mathrm{AMst}^{\mathrm{ed}}$, sicut proposuit corde $757689 \mathrm{SPM}$, lac. 88*, secundum propositum cordis sui $\mathrm{AMst}^{\mathrm{AD}}$, secundum propositum cor AMst ${ }^{\text {var }}$, sicut corde proposuit TES ${ }^{\text {ed }}$, sic aut corde proposuit TES ${ }^{\text {var }}$

non ] lac. 88*, ut non $\mathrm{AMst}^{\mathrm{AD}}$; + quasi TES $\mathrm{Sd}^{\mathrm{ed}}$

ex tristitia aut $5154(89)$ ] ex tristitia uel TES $S^{\text {ed }}$

ex necessitate $A M s t^{\text {var }} \mathrm{SPM}^{\mathrm{var}}$ ] necessitate $617576 \mathrm{AMst}^{\text {ed }} \mathrm{PEL}^{\mathrm{var}} \mathrm{SPM}^{\mathrm{ed}}$, necessitatem 89 SPM $^{\text {var }}$ + agerent $\mathrm{AMst}^{\mathrm{AD}}{ }^{*}$

hilarem enim datorem 5154 ] hilarem dolorem $\mathrm{TES}^{\text {var }}$

diligit 61 ] dicit $75^{*}$, eligit $S \mathrm{PM}^{\mathrm{var}}$

deus ] om. PEL var, dominus TES ${ }^{\text {var }}$

9:8 Present: $5^{1} 545^{86175767778} 88$ 89 AMst PEL SPE

potens est ] potest 777888 , potens 89

autem deus ] deus $\mathrm{AMst}^{\mathrm{R}}$, enim deus PEL var

omnem ] om. AMst var

gratiam 88 ] gloriam 58 , scientiam gratiam $\mathrm{PEL}^{\mathrm{A} *}$

abundare 515861 ] abunda[1-3] 88*

facere AMst ${ }^{\mathrm{var}}$ ] om. 75767789 AMst $^{\mathrm{ed}}$

in uobis ] in nobis PELvar

ut in omnibus AMst ${ }^{\text {var }}$ ] ut ubique 7576 , ubique 89, in omnibus AMst ed $^{\text {ed }}$ semper ] om. 77 PELvar

omnem sufficientiam 54 ] sufficientiam 61

habentes ] habeatis 61; + et 61, + ut AMst ${ }^{\text {ed }}$

abundetis $5^{1} 5^{861}$ ] abundemus $P E L^{B}$, om. PEL ${ }^{\text {var }}$ 
in omne ] ad omne 89

opus bonum ] bonum opus 61, opere bono AMstvar

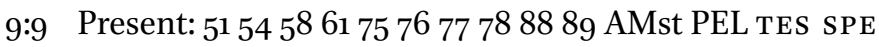

sicut ] sicuti TES ${ }^{\mathrm{var}}$, om. TES ${ }^{\mathrm{var}}$; + in psalmo cxi 61

scriptum est

dispersit ] sparsit 7576 , distribuit TES

dedit pauperibus iustitia eius $515488^{C}(89)$ ] dedit pauperibus $88^{*}$

manet in

aeternum 54 ] saeculum saeculi $5158617778 \mathrm{AMst}^{\mathrm{var}} \mathrm{TES}^{\mathrm{var}}$, saeculum $\mathrm{AMst}^{\mathrm{A}}$

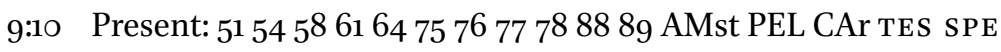
qui autem ] lac. 64, qui CAr TES

administrat ] ministrat $54^{*} 89 \mathrm{AMstr}^{\mathrm{var}}$, subministrat $6175767788^{*} \mathrm{AMst}^{\mathrm{ed}}$ CAr, lac. 64

semen PEL ${ }^{\mathrm{var}}$ ] lac. 64, om. PEL var

seminanti ] lac. 64, seminat $\mathrm{TES}^{\mathrm{var}}$, seminante $\mathrm{TE} \mathrm{S}^{\mathrm{var}}$, seminantis $\mathrm{TES} \mathrm{S}^{\mathrm{var}}$ et panem ] lac. 64

ad manducandum $\left.\mathrm{AMst}^{\mathrm{var}} \mathrm{TES}^{\mathrm{var}}\right]$ in escam $617576 \mathrm{CAr}^{\mathrm{ed}}$, lac. 64, ad uel in escam manducandum 77 , et [...] $88^{*}$, ad manducan [2-3] $89^{*}$, ad edendum AMsted ${ }^{\text {TES }}{ }^{\text {ed }}$, in esca $P E L^{\mathrm{B}}$, ab esca CArvar, et ad edendum TES $^{\mathrm{var}}$

praestabit $5154587888^{c} \mathrm{AMst}^{\mathrm{var}}$ ] ministrabit 61 AMsted, lac. 64, subministrauit $7576 \mathrm{CAr}^{\mathrm{var}}$, ministrabit uel tribuet 77 , om. $88^{*}$, tribuet 89 , subministrabit PEL ${ }^{\mathrm{B}} \mathrm{CAr}^{\mathrm{ed}}$, administrabit TES ${ }^{\mathrm{var}}$, tribuit TES $\mathrm{S}^{\mathrm{var}}$

et multiplicabit ] lac. 64, et multiplicauit 5489 AMst $^{\mathrm{var}} \mathrm{PEL}^{\mathrm{var}} \mathrm{TES}^{\mathrm{var}} \mathrm{SPE}^{\mathrm{var}}$, et multiplicabit $88^{*}$, abs. CAr

semen uestrum TES $\left.{ }^{\text {var }}\right]$ lac. $64, \operatorname{semin}\left[5^{-7}\right]$ uestram $88^{*}$, seminationem uestram $\mathrm{TES}^{\mathrm{ed}}$, abs. CAr

et augebit 64 ] et amplificabit AMsted, et amplificauit AMstrar, et ampliabit $\mathrm{AMst}^{\mathrm{var}} \mathrm{PEL}^{\mathrm{B}}$, abs. CAr; + uel ampliabit 77

incrementa frugum ] opera 61 , nascentes fruges 64 , nascentia 757689 , opera uel nascentia 77 , fruct $[1] 88^{*}$, fructum AMst, incrementum frugum PEL ${ }^{\text {var }}$, frugum PEL ${ }^{\mathrm{var}}$, incrementa TES ${ }^{\mathrm{var}}$, incrementum fructum TES ${ }^{\mathrm{var}}$, abs. CAr iustitiae uestrae $51545861\langle 64\rangle 778889]$ abs. CAr

9:11 Present: 51545861647576777888 89 AMst PEL tes SPE

ut ] om. 6475768889 , et TES ${ }^{\text {var }}$

in omnibus ] + uos 61

locupletati (51) ] ditati 64, om. 88*, locupletemini AMst TES ${ }^{\text {ed }}$, locupletimini TES var 
abundetis $51586188^{c}$ ] om. $6475767788^{*} 89$ AMst, abs. TES

in omnem simplicitatem $\langle 64\rangle(89) \mathrm{AMst}^{\mathrm{R}}$ ] in omni simplicitate $61 \mathrm{AMst}^{\mathrm{ed}}$, $a b s$. TES

quae operatur $\left.51545^{8} 7888 \mathrm{AMst}^{\mathrm{var}}\right]$ si quis operatur $89 \mathrm{AMst}^{\mathrm{ed}}$, abs. TES per $\langle 64\rangle]$ om. PEL ${ }^{\text {var }}$, abs. TES

nos ] ụos 51, lac. 64, uos $77^{*}$, abs. TES

gratiarum actionem 515488 ] gratiam actionem 89, abs. TES

deo ] domino 64 , om. $88^{*}$, abs. TES

9:12 Present: $5^{1} 545^{8} 6164757677788889$ AMst PEL TES SPE

quoniam $\langle 64\rangle$ ] quia 75767789 , abs. TES

ministerium $\langle 64\rangle$ ] ministratio 757677 , ministrationem 89, administratio TES

huius officii ] administrationis officii huius 61, om. 64 88*, officii huius 7576 $\mathrm{AMst}^{\mathrm{var}}$, officii uel administrationis huius 77, officii eius $89 \mathrm{AMst}^{\mathrm{ed}}$, administrationis huius officii $\mathrm{PEL}^{\mathrm{B}}$

non solum ] non tantum TES

supplet ea 586488 ] replens ea 7576 , supplet uel est ad supplenda ea 77 , ea supplet 78 , replens est ea 89 , supplebit ea TES ${ }^{\text {ed }}$, impleuit ea TES ${ }^{\text {var, }}$, suppleuit TES ${ }^{\text {var }}$ + et TES ${ }^{\text {var }}$

quae $5154^{c} 58617788$ ] q[1] $54^{*}$, lac. 64

desunt sanctis TES ${ }^{\text {var }}$ ] sanctis desunt TES ${ }^{\text {ed }}$

sed etiam ] sed 64 TES $^{\text {var }}$, sed et $75767788^{*}$ AMst TES ${ }^{\text {ed }}$

abundat $51586188^{C} \mathrm{AMst}^{\mathrm{R}}$ ] abundare faciet 64 , abunda [1] $88^{*}$, om. 89, abundabit $A M s t^{\text {ed }}$ TES $^{\text {ed }}$, abundauit AMst ${ }^{\mathrm{var}} \mathrm{TES}^{\mathrm{var}}$, abundet $\mathrm{SPE}^{\mathrm{var}}$

per ] lac. 64

multas ] multos 51, multarum 6164757677 AMst $^{\text {var }}$ PEL $^{\text {B }}$ TES ${ }^{\text {var }}$, multa[3] $88^{*}$, multorum AMst ${ }^{\text {ed }}$, multam TES ${ }^{\text {ed }}$, multa TES ${ }^{\text {var }}$

gratiarum 88 ] om. $64^{*}$

actiones $5488^{c} \mathrm{AMst}^{\mathrm{R}}$ ] actione $5^{1}$ 64, actionem $75767788^{*} \mathrm{AMst}^{\text {ed }} \mathrm{PEL}^{\mathrm{B}}$ TES, actionum $\mathrm{AMst}^{\mathrm{A} * \mathrm{D} *}$

in domino $\mathrm{TES}^{\mathrm{var}}$ ] deo $616475767788^{*} 89 \mathrm{AMst}^{\mathrm{ed}} \mathrm{PEL}^{\mathrm{B}}$, in deo $88^{\mathrm{C}} \mathrm{AMst}^{\mathrm{var}}$, in domino deo $\mathrm{TES}^{\mathrm{var}}$, in deum $\mathrm{TES}^{\mathrm{ed}}$

9:13 Present: $5^{1} 545^{861} 647576777888$ 89 AMst PEL sPE

per probationem $\langle 64\rangle 88$ ] per experimentum 6189 , per expedimentum 75 76

ministerii ] ministrationis 64757689 , minist [5-6] 88*

huius glorificantes $\langle 64\rangle]$ huius magnificantes 6175767789 AMst PEL $^{\mathrm{B}}$

deum ] lac. 64, dominum 89 PEL $^{\text {var }}$; + in uestram 89 
oboedientia $5154586188^{c}$ ] in obsequio 64 , in obaudientiam 7576 , in subiectione $77 \mathrm{AMst}^{\mathrm{ed}}$, in ob $\left[5^{-7}\right] 88^{*}$, ị confestinationem 89 , in subiectionem $\mathrm{AMst}^{\mathrm{var}} \mathrm{PEL}^{\mathrm{B}}$, in obaudientia PEL ${ }^{\mathrm{var}}$, in oboedientiam $\mathrm{SPE}^{\mathrm{var}}$

confessionis uestrae 54587788 ] confessionis ṇostrae 5 , per oboedientiam 89

in euangelium christi $54\langle 64\rangle$ ] in euangelio christi $5861757^{6} 88^{\mathrm{C}} 89$ AMst PEL $^{\text {var, }}$, in angelum christi PEL ${ }^{\text {var }}$, om. SPE et $\mathrm{AMst}^{\mathrm{R}}$ ] om. $89 \mathrm{AMst}^{\mathrm{ed}}$; + in 617789 AMst simplicitate 61 ] simplicitatem $64\left\langle 88^{*}\right\rangle \mathrm{SPE}^{\mathrm{var}}$ communicationis $61\langle 64\rangle]+$ uestrae $78 \mathrm{AMst}^{\mathrm{ADR}} \mathrm{PEL}^{\text {var }}$ in illos ] in ipsos $\mathrm{AMst}^{\mathrm{R}}$, om. $\mathrm{AMst}^{\mathrm{var}}$, in illo PEL var, illius $\mathrm{SPE}^{\mathrm{var}}$; + sit PEL $\mathrm{PLr}^{\mathrm{var}}$ et in omnes $\langle 64\rangle \mathrm{AMst}^{\mathrm{R}}{ }^{\text {] om. }} 6189 \mathrm{AMst}^{\mathrm{ed}}$, in omnes PEL var

9:14 Present: 5154586164757677788889 AMst PEL SPE

et ] lac. 64; + in 51546164 AMst $^{\text {var }}$

ipsorum ] eorum 757689

obsecratione ] praedication[em] 64, obsecrationem $75767889 \mathrm{PEL}^{\mathrm{var}}$, oratione $77 \mathrm{AMst}^{\mathrm{AR}} \mathrm{PEL}{ }^{\mathrm{var}}$, precatione $88^{*}$, orationes $\mathrm{AMst}^{\mathrm{ed}}$, obsecrationes $\mathrm{PEL}^{\mathrm{B}}$, om. PEL var

pro uobis $\langle 64\rangle]$ pro ụobis 58

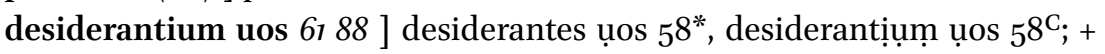
uidere AMst

propter eminentem 89 ] propter superabundantem $6_{1} A M s t^{e d} \mathrm{PEL}^{\mathrm{B}}$, propter excellentem $\langle 64\rangle 88^{*}$, propter abundantem $A M s t^{A}$; + uel excellentem 77 gratiam dei 88 ] gloriam dei $545^{8}$

in uobis ] in ụobis 58, om. 64

9:15 Present: $5^{1} 545^{8} 6164757677788889$ AMst PEL SPE

gratias $88 \mathrm{PEL}^{\text {var }}$ ] gratia 757689 ; + ago ${ }_{51} 6{ }_{1} 64 \mathrm{PEL}^{\mathrm{B}} \mathrm{SPE}^{\mathrm{ed}},+$ autem $78 \mathrm{AMst}^{\mathrm{R}}$ PEL ${ }^{\mathrm{A}}$

deo ] + meo PEL ${ }^{B}$

super $\langle 64\rangle$ ] per AMst ${ }^{\mathrm{var}}$, semper SPE ${ }^{\mathrm{var}}$

inenarrabili $545^{8}\langle 64\rangle$ ] enarrabili $61757_{6} 88^{*} \mathrm{PEL}^{\mathrm{B}}$, inenarrabilia 89, ineffabili AMst ${ }^{\mathrm{R}}$, inenarrabile PEL ${ }^{\text {var }}$, enarrabile $\mathrm{PEL}^{\mathrm{var}} \mathrm{SPE}^{\mathrm{var}}$

dono eius ] deo nostro 64, eius dono 757677 , dona eius 89, donum eius PEL var

10:1 Present: 51545861647576777888 89 AMst PEL ipse autem ego paulus $\left(75^{*}\right)$ ] ipse ego $p$ [aulus] 64 obsecro uos per mansuetudinem et $61\langle 64\rangle$ 
modestiam christi ] oboedientiam christi PEL ${ }^{\text {var }}$

qui ] qui uel quae 77 , quia $89 \mathrm{AMst}^{\mathrm{var}} \mathrm{PEL}^{\mathrm{var}}$

in ] ad 647576 AMst, ad uel secundum 77 , lac. 88*

facie quidem ] faciem quidem $\langle 64\rangle 757^{6} 77\left\langle 88^{*}\right\rangle$ 89 AMst PEL ${ }^{\text {var }}$

humilis ] om. PEL var; + sum $58617^{\mathrm{C}}$ AMst PEL ${ }^{\mathrm{B}}$

inter uos $\mathrm{AMst}^{\mathrm{R}}$ ] in uobis $6488^{*} \mathrm{AMst}^{\mathrm{ed}}$, om. 89 ; + humilis PEL var

absens autem ] absens uero $\mathrm{AMst}^{\mathrm{R}}$

confido in uobis $\langle 64\rangle$ ] audeo in uobis 7576 , audeo in uos 89

10:2 Present: 5154586164757677788889 AMst PEL

rogo autem ] precor autem $6488^{*}$, obsecro autem 75 76, om. 89; + uos $\mathrm{AMst}^{\mathrm{R}}$

ne ] ut non 61757677 AMst PEL ${ }^{\mathrm{B}}$, ut ne 89

praesens $51545861\langle 64\rangle 7788$

audeam ] confidentius agam 64 , lac. $88^{*}$

per eam $\langle 64\rangle \mathrm{AMst}^{\mathrm{R}}$ ] om. 757689 , per $77 \mathrm{AMst}^{\mathrm{ed}} \mathrm{PEL}^{\mathrm{B}}$

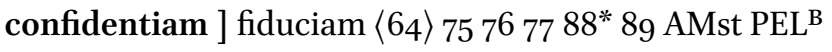

qua ] quam $5^{1} 5464$, qu[...] 88*, quae $89 \mathrm{PEL}^{\text {var }}$, quia AMstvar $\mathrm{PEL}^{\mathrm{A}}$; + me habere 64

existimo ] existimor $51545^{8617576} 777^{8} 88^{\mathrm{C}} \mathrm{AMst} \mathrm{Aar}^{\mathrm{var}} \mathrm{PEL}$, lac. $88^{*}$, aestimor AMst ${ }^{\text {ed }}$, exaestimor $A M s t^{A D}$

audere ] habere 51 , audet 54, om. $64 \mathrm{AMst}^{\mathrm{var}}$, audire $7576 \mathrm{AMst}^{\mathrm{var}} \mathrm{PEL}^{\mathrm{var}}$, lac. $88^{*}$

in quosdam $\langle 64\rangle \mathrm{AMst}^{\mathrm{R}}$ ] lac. $88^{*}$, in aliquos $\mathrm{AMst}^{\mathrm{ed}} \mathrm{PEL}^{\mathrm{B}}$

qui arbitrantur ] arbitrantes uel existimantes 77 , lac. $88^{*}$, qui arbitrabantur AMst $^{\text {var }}$

nos ] de nobis 6177 , lac. $88^{*}$

tamquam 77 ] quas [i] 64, lac. $88^{*}$, quod tamquam $89 \mathrm{PEL}^{\mathrm{B}}$, om. AMst

secundum carnem $\langle 64\rangle$

ambulemus ] militamus 61 , ambulantes $7788^{*}$, ambulamus $\langle 64\rangle 88^{\mathrm{C}}$, ambulare AMst

10:3 Present: $51545^{8} 61647576777888$ 89 AMst PEL SPM

in carne enim $\langle 64\rangle]$ in carne autem ${ }_{51}^{1}$, nam in carne 61 AMst $^{\text {ed }}$, in carnem enim 89, non in carne AMstvar

ambulantes ] ambulamus AMst ${ }^{\mathrm{var}}$ non secundum carnem $\langle 64\rangle\left(88^{*}\right)$

militamus 64 ] militantes AMstvar 
10:4 Present: $5^{1} 545^{8} 6164757677788889$ AMst PEL SPM nam arma ] arma enim 61 $\langle 64\rangle 757677$ AMst PEL ${ }^{B}$ SPM, arma [4] 88* militiae nostrae non $515458\langle 64\rangle 77(88)$ carnalia ] sunt carnalia 58 , carnalia sunt $\mathrm{AMst}^{\mathrm{R}}$ sed potentia $54\langle 64\rangle]$ s sed fortia $6177 \mathrm{AMst}^{\mathrm{PEL}}{ }^{\mathrm{B}}$, sed potentiae PELvar deo ] a deo $5^{861}$, dei AMst ${ }^{\text {var }}$

ad destructionem $61\langle 64\rangle 7888^{*}$ ] ad destruendam $7576 \mathrm{SPM}^{\mathrm{var}}$ munitionum consilia ] munitio[3] consilia 64, munitionem consilia 7576 $88^{*} \mathrm{SPM}^{\mathrm{var}}$, qui in superbia sensus gloriantur 89 , munitionum cogitationes AMst, inimicorum cogitationes $\mathrm{PEL}^{\mathrm{B}}$ destruentes 6178 ] destruens AMstrar

10:5 Present: $5^{1} 545^{8} 6164757677788889$ AMst PEL sPM et ] om. 89

omnem altitudinem SPM ${ }^{\mathrm{var}}$ ] lac. 64 , omnem exaltationem $89 \mathrm{PEL}^{\mathrm{var}} \mathrm{SPM}^{\mathrm{ed}}$ extollentem ] lac. 64, om. PELvar

se aduersus ] lac. 64, aduersus 77 , sed aduersus $\mathrm{SPM}^{\mathrm{var}}$ scientiam ] lac. 64, cognitionem $\mathrm{AMst}^{\mathrm{R}}$, cogitationem AMst ${ }^{\mathrm{ed}}$ dei ] christi 61, lac. 64 et $\mathrm{AMst}^{\mathrm{R}}$ ] om. 6175767789 AMst $^{\mathrm{ed}} \mathrm{PEL}^{\mathrm{B}}$ SPM, lac. 64 in captiuitatem redigentes ] captiuantes $6175767788^{*} \mathrm{PEL}^{\mathrm{B}}$, lac. 64, captiuum ducentes 89

omnem intellectum SPM${ }^{\mathrm{var}}$ ] lac. 64 , omnem cogitationem $\mathrm{SPM}^{\mathrm{ed}}$; + et AMst $^{\text {ed }}$

in obsequium ] ad oboediendum $617789 \mathrm{PEL}^{\mathrm{B}}$, lac. 64, ad obaudiendum 75 76 , [10-14] 88*, in obsequio $88^{\mathrm{C}}$, ad obaudientiam AMst; + uel in oboedientiam 77

christi ] christo $617576 \mathrm{PEL}^{\mathrm{B}}$, lac. 64, christo uel christi 77 , domino 89 \pm perducentes $6175767789 \mathrm{AMst}^{\mathrm{ed}} \mathrm{SPM}^{\mathrm{ed}}$, lac. 64, perducere SPM ${ }^{\mathrm{var}}$

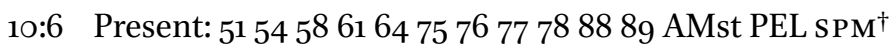

et in promptu habentes $54^{*} \mathrm{SPM}^{\mathrm{var}}$ ] et in prompto habentes 58 , et parati omnem 61, lac. 64, et promptu habentes 77 , et [8] habentes $88^{*}$, et parati $\mathrm{AMst}^{\mathrm{ed}}$, et parati in omnibus $\mathrm{AMst}^{\mathrm{R}}$, et parat $\mathrm{AMst}^{\mathrm{var}}$, et in promptum habentes $P E L^{\text {var }}$

ulcisci ] om. 61, lac. 64, uindicare 75767789 AMst, ulciscionem PEL ${ }^{\mathrm{B}}$

$\dagger 2$ Cor. 10:6. Only the first four words of this verse are present in SPM as a variant continuing from the previous verse. 
omnem AMst ${ }^{\mathrm{R}}$ ] om. $61 \mathrm{PEL}^{\mathrm{B}}$, lac. 64, in omnem 89, in omnibus AMsted

inoboedientiam $5154586188 \mathrm{AMst}^{\mathrm{R}}$ ] [...] entiam 64, inobaudientiam 7576 AMst $^{\text {ed }}$ PEL $^{\text {var }}$, oboedientiam $7889 ;+$ uindicare 61

cum impleta 61 ] cum conpleta 6488

fuerit ] fuerint $\mathrm{PEL}^{\mathrm{var}}$; + prior $\langle 64\rangle 88$

uestra oboedientia $51545^{8}$ ] oboedientia nostra 6189 , oboedientiam ueram

$\langle 64\rangle$, obauditio nostra 7576 AMst $^{\text {ed }}$, oboeditio nostra 77 , oboedientiam uestram $88^{*}$, oboedientia uestra $88^{\mathrm{C}} \mathrm{AMst}{ }^{\mathrm{var}}$, obauditio uestra $\mathrm{PEL}^{\mathrm{B}}$, uestra obaudientia PELvar

10:7 Present: 5154586164757677788788 89 AMst PEL CAr SPM

quae secundum $51545^{8} 6176777888$ ] abs. 87 CAr; quae autem secundum SPM $M^{\text {var }}$

faciem sunt $88^{c}$ ] personam sunt $\langle 64\rangle 88^{*}$, abs. $87 \mathrm{CAr}$

uidete ] cauete $\mathrm{SPM}^{\mathrm{var}}$, abs. 87

si quis ] fratres si quis 87, abs. SPM

confidit sibi ] confidet 7576 , confidet sibi 7789 PEL $^{\text {var }}$, confidit 78 AMst, sibi confidit $\langle 64\rangle 88$, abs. SPM

christi se esse 54 ] christi seruum se esse 58 PEL $^{\text {var }}$, esse seruum christi 61 $\mathrm{PEL}^{\mathrm{B}}$, se christi seruum esse 757678 , christi seruus esse 77 , christi esse $\langle 64\rangle 88$, se christi esse 89, esse se seruum christi AMst ${ }^{\text {ed }}$, esse se christi $\mathrm{AMst}^{\mathrm{R}}$, se esse seruum christi $\mathrm{AMst}^{\mathrm{var}}$, om. CAr, abs. SPM; + iterum $5^{1}$

hoc cogitet ] hoc exaestimet $89^{*}$, hoc existimet $89^{\mathrm{C}}$, hoc cogitat $\mathrm{AMst}{ }^{\mathrm{var}}$, abs. SPM

iterum ] om. $5164^{*}$, item PEL ${ }^{\text {var }}$, abs. SPM

apud se $5158647587\langle\mathrm{CAr}\rangle]$ intra se $6177, a b s$. SPM

quia sicut ] quia [quom] odo 64, lac. 88*, abs. CAr SPM; + et 54

ipse christi est ] ipse christi 77, abs. CAr SPM

ita et nos ] sic et nos $6488^{*}$, ita nos PEL ${ }^{\text {var }}$, abs. CAr SPM

10:8 Present: 5154586164757677788788 89 AMst PEL

nam et si ] si enim $61757677 \mathrm{PEL}^{\mathrm{B}}$, siue enim 64, nam si $8789 \mathrm{AMst}^{\mathrm{var}}$, lac. 88*, nam si et PEL var

amplius aliquid 54 ] abundantius aliquid 61 , [abun] dantius 64, aliquid abundantius 757677 , aliquid amplius 87 AMst PEL ${ }^{\mathrm{A}}$, lac. 88*

gloriatus ] glorificatus $5^{1} 64 \mathrm{AMst}^{\mathrm{var}}$, gloriabor uel gloriatus 77

fuero de potestate nostra quam (64) 87 ] fuero de potestate uestra quam PELvar 
dedit dominus ] nobis dedit dominus 58 88*, nobis dedit deus $6164 \mathrm{PEL}^{\mathrm{B}}$, dedit deus 7576 , dedit deus nobis 77 , dedit nobis deus $78 \mathrm{PEL}^{\text {var }}$, dedit nobis dominus AMst

in ] et in 7576 , ad 78, om. PEL var

aedificationem $515458\langle 64\rangle 768788$ ] aedificatione AMstrar, om. PEL var

et non in ] et in $51,0 m .54^{*}$ non in AMsted, non AMst ${ }^{\text {var }}$

destructionem $61\langle 64\rangle(75) 78(87) 88$ ] om. $54^{*}$

uestram $\mathrm{AMst}^{\mathrm{AD}}$ ] uestri AMsted

non ] ut non $\mathrm{PEL}^{\mathrm{B}}$

erubescam ] confundar 757689 , erubescant AMst ${ }^{\mathrm{var}}$

10:9 Present: $51545^{8} 6164757677788788$ 89 AMst PEL

ut autem $\mathrm{AMst}^{\mathrm{R}}$ ] ut $647576778788^{*} 89 \mathrm{AMst}^{\text {ed }} \mathrm{PEL}^{\mathrm{B}}$

non ] modo 51

existimer $\langle 64\rangle$ ] existimemur 61757677 AMsted exaestimetur 89*, existimetur $89^{\mathrm{C}}$, exaestimur AMst ${ }^{\mathrm{var}}$

tamquam $\langle 76\rangle 78$ ] quasi (77) $\mathrm{AMst} \mathrm{PEL}^{\mathrm{B}}$

terrere $\mathrm{AMst}^{\mathrm{R}}$ ] terrentes $61757689 \mathrm{AMst}^{\text {ed }} \mathrm{PEL}^{\text {var }}$, terrendo uel terrentes

77 , terrens $\left(\mathrm{PEL}^{\mathrm{B}}\right) \mathrm{PEL}^{\text {ed }}$

uos per epistulas ${ }_{51} 61\langle 64\rangle 767778878889$

10:10 Present: 5154586164757677788788 89 AMst PEL

quoniam ] quia $\langle 64\rangle$ AMst, quo 76 , lac. $88^{*}$

quidem epistulae inquiunt $5154586177788788^{c}$ ] epistulae quidem aiunt

64, quidem epistulae inquit 7576 , lac. $88^{*}$, epistulae quidem $89 A M s t^{e d}$, epistulae quidem inquiunt $A M s t^{R}$, iam aiunt epistulae $P E L^{\text {var }}$

graues $\langle 64\rangle$ ] gẹnțẹs graues 51 , graue 54

sunt et fortes ] et fortes sunt $61\langle 64\rangle 757688^{*} \mathrm{AMst}^{\mathrm{R}} \mathrm{PEL}^{\mathrm{A}}$, et fortes $77 \mathrm{AMst}^{\mathrm{ed}}$ praesentia 51545861778788 ] praes[1] entia $89^{*}$

autem corporis infirma et $\langle 64\rangle$ ] uero corporis infirma et 89

sermo contemptibilis $\left.\langle 64\rangle\left(88^{*}\right)\right]$ sermo contemptibilis suus AMst ${ }^{\text {var }}$; + uel infacundus 77

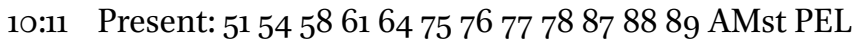

hoc PEL var ] hoc ergo $51545^{\mathrm{C}} 78 \mathrm{PEL}^{\mathrm{AB}}$, ergo hoc $58^{*}$

cogitet qui ] aestimet qui AMsted, aestimet quia $\mathrm{AMst}^{\mathrm{var}}$

eiusmodi $\mathrm{AMst}^{\mathrm{AR}}$ ] huiusmodi 61 $\mathrm{AMst}^{\mathrm{t}}$, huiuscemodi $\mathrm{AMst}^{\mathrm{var}}$, eius est modi PEL var

est ] lac. $6489^{*}$, om. AMstvar PEL ${ }^{\text {var }}$

quia quales sumus 87 ] quoniam quales sumus 6488 
uerbo ] sermone 89

per epistulas absentes $\left.{ }_{51} 61\langle 64\rangle 7677\right\rangle 8878889$

tales ] om. 87 ; + sumus $64757688^{*}$ AMst PEL var

et praesentes $51545^{861}\langle 64\rangle 7788$ ] om. 8789 ; + sumus $5^{8} 6189 \mathrm{PEL}^{\mathrm{B}}$

in facto ] foris in facto 58 , in opere $61 \mathrm{AMst}^{\mathrm{ed}} \mathrm{PEL}^{\mathrm{B}}$, opere $6488^{*} \mathrm{AMst}^{\mathrm{var}}$, facto 77 , et facto praesentes 89

10:12 Present: 5154586164757677788788 89 AMst PEL

non enim audemus ] non enim audimus 7576 , non audemus enim 78 ; + uos $5^{8}$

inserere ] conferre $61777^{8} \mathrm{AMst}^{\mathrm{R}} \mathrm{PEL}^{\mathrm{B}}$, [adop] tare 64, coniungere 757689 AMst $^{\text {ed }}$, [3-5] ${\text { ere } 88^{*}}^{*}$

aut conparare nos $58778788^{c}$ ] nos aut conparare 7576 , aut conparare nos [6-8] $88^{*}$, aut conparare nosmetipsos $A M s t^{e d}$, aut conparare nosip$\operatorname{sos} A M s t^{R}$

quibusdam qui se ipsos $\left.\langle 64\rangle \mathrm{AMst}^{\mathrm{var}}\right]$ quibusdam qui semet ipsos $757^{6}$ AMsted, quibusdam se ipsos 77

commendant ] commendantibus 77

sed ipsi in ] sed ipsi intra $757^{2} \mathrm{PEL}^{\mathrm{B}}$, sed ipsi inter 89

nobis AMstvar ${ }^{\mathrm{va}}$ om. $5^{1} 5^{8} 757^{6} 89 \mathrm{AMst}^{\mathrm{ed}} \mathrm{PEL}^{\mathrm{B}}$, se[met] ipsis 64, uobis 78 , lac. $88^{*}$

nosmet ipsos ] nobismet ipsos $58 \mathrm{AMst}^{\mathrm{ed}}$, semet ipsos $64,[\ldots]$ met ipsos $88^{*}$, nọ̣met ipsos $88^{\mathrm{C}}$, uobismetipsis AMstvar

metientes ] mentientes $515487\left\langle 88^{*}\right\rangle$, om. 64 , misurantes 7576 , mensurantes 77 AMst, mortificantes 89

et conparantes 587677788788

nosmet ipsos nobis $\langle 51\rangle]$ [sem] et ipso[s] sibimet ipsis 64 , nobis nosmet ipsos 7576 , nosmet ipsos sibi $87,[\ldots]$ met ipsos [...] 88*, nobismet ipsos 89 , nosmet ipsos uobis $\mathrm{PEL}^{\mathrm{B}} ;+$ non intelle $[$ gun $] \mathrm{t} 64,+[\ldots]$ 88*

10:13 Present: 5154586164757677788788 89 AMst PEL

nos autem non ] non 617576777889 AMst, nos uero non $\langle 64\rangle$ 88*, non autem PEL $^{\mathrm{B}}$, nos autem PEL ${ }^{\text {var }}$

in inmensum $\left.58757678 \mathrm{PEL}^{\mathrm{var}}\right]$ inmensum ${ }_{51} 87 \mathrm{PEL}^{\mathrm{A}}$ *

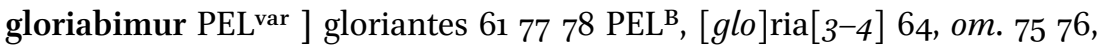
gloriemur 89, gloriabitur AMstvar, gloriamur $\mathrm{PEL}^{\mathrm{A}}$

sed secundum (87) ] sed per PEL ${ }^{\text {var }}$

mensuram regulae 515458778788 ] mensuram [ glo]riae 64

quam mensus ] qua mensus $5^{8} 77^{8 \mathrm{C}} 89$, quam emensus 61 , quam partitus 64 AMst, quam $[6-8] 88^{*}$ 
est nobis deus ] est deus nobis 61, est nobis dominus 7576 , est deus 77 mensuram $\langle 64\rangle]$ mensura 89, om. PEL var pertingendi $6 r\langle 64\rangle]$ ] contingendi 7576 , continentiae 89

usque ] + et 757677 ad uos ] ad nos $\mathrm{PEL}^{\mathrm{B}}$

10:14 Present: $5^{1} 545^{8} 616475767778878889$ AMst PEL non enim quasi non pertingentes 61 ] om. 64

ad uos ] usque ad uos $5^{1} 61 \mathrm{AMst}^{\mathrm{PEL}} \mathrm{L}^{\mathrm{B}}$, om. 64, ad uel in uos 77 superextendimus nos $\langle 64\rangle 87$ ] superextendimus uos $5^{8}$

usque ] ipsos usque 64 , usque et 7576 , usque enim et 77, [2-4] usque $88^{*}$, nam usque $\mathrm{AMst}^{\mathrm{ed}}$, nam et usque AMst ${ }^{\mathrm{var}}$, usque enim PEL ${ }^{\text {var }}$ ad uos ] ad nos 58 , ad [...] 64 enim AMst ${ }^{\text {var }}$ ] om. 77 AMst $^{\text {ed }}$ PEL var peruenimus ] pertingimus 64, perueniamus PEL var in euangelio christi 545861

10:15 Present: $5^{1} 545^{8} 6164757677788488$ 89 AMst PEL non ] lac. 64, abs. 84; + enim 89 in ] om. 51 $54^{*} 61757688^{*}$ AMst $^{\text {var }}$ PEL ${ }^{\text {var }}$, lac. 64, abs. 84 inmensum $58\langle 64\rangle 757^{6} 78$ ] inmensa $77, a b s$. 84, inmenso 89 gloriantes in alienis laboribus $\langle 64\rangle]$ abs. 84 spem autem ] spero autem 51, lac. 64, fratres spem 84, sed spem 89, spem uero AMst

habentes ] lac. 64

crescentis ] crescentes $54 \mathrm{AMst}^{\mathrm{var}}$, crescente 61 PEL ${ }^{\mathrm{var}}$, lac. 64, in crescente 75767789 , crescent $[\ldots]$ 84, crescenti PEL ${ }^{\mathrm{B}}$, credentes PEL ${ }^{\mathrm{var}}$

fidei uestrae $51545^{8} 88$ ] fide uestra 61757677 (89) PEL var, lac. 64, abs. 84, fidei nostrae $P E L^{B}$

in uobis ] lac. 64, in nobis $757678 \mathrm{AMst}^{\mathrm{var}}$, abs. 84 magnificari ] lac. 64, abs. 84, magnificare AMst ${ }^{\mathrm{AD}} \mathrm{PEL}^{\mathrm{var}}$ secundum ] lac. 64, abs. 84, om. 89, per PELvar regulam nostram ] nostram regulam 61, lac. 64, abs. 84

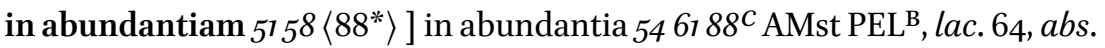
84

10:16 Present: 51545861647576777888 89 AMst PEL etiam PEL var $]$ lac. 64, om. 75767789 AMst $^{\text {ed }}$ PEL $^{\mathrm{AB}}$, et ut etiam AMst $^{\mathrm{R}}$ in illa quae 51545888 ] illis gentibus quae 61, lac. 64, om. 75767789 , in regionibus quae $\mathrm{AMst}^{\mathrm{ed}}$, regionibus quae $\mathrm{AMst}^{\text {var }}$ 
ultra ] lac. 64, om. 89, ultro AMst ${ }^{\mathrm{var}}$ uos ] ṇos 51, lac. 64, om. 89, nos PEL ${ }^{\mathrm{B}}$ sunt ] om. 7576777889 ; + in aliis gentibus $5^{8}$ euangelizare 545861 ] om. 89, euangelizem ut $\mathrm{AMst}^{\mathrm{R}}$ non in ] non $7576 \mathrm{PEL}^{\mathrm{var}}$, om. $\mathrm{PEL}^{\mathrm{B}}$ aliena regula ] alienam regulam 64 , aliena regula $[1] 88^{*}, o m . \mathrm{PEL}^{\mathrm{B}}$ in his 58 ] in eam 64, in 757677 , in ea 89, om. AMst quae praeparata sunt 5154586178 ] praeparata 7576 77, q[1] praeparatae sunt $88^{*}$, quae praeparatae sunt $88^{\mathrm{C}}$, quae praeparati sunt 89 , praeparatis $\mathrm{AMst}^{\text {ed }}$, praeparatus $\mathrm{AMst}^{\mathrm{var}}$, quae praecepta sunt $\mathrm{PEL}^{\mathrm{var}}$ gloriari ] gloriemur $\mathrm{AMst}^{\mathrm{R}}$, ad gloriam $\mathrm{PEL}^{\mathrm{B}}$

10:17 Present: $51545^{8} 61647576777888$ 89 AMst PEL SPE \pm in hieremia enim ait 61 qui autem $\langle 64\rangle$ ] nam qui 757689 ; + in domino $\mathrm{PEL}^{\mathrm{B}}$ gloriatur in domino glorietur ] gloriatur 7576

10:18 Present: 51545861647576777888 89 AMst PEL SPE non enim ] non uel nec enim 77 qui se ipsum $\mathrm{AMst}^{\mathrm{var}}$ ] qui semet 76 , qui se $\mathrm{AMst}^{\mathrm{ed}}$ commendat 5888 ] + et AMstrar ille $\mathrm{AMst}^{\mathrm{R}} \mathrm{PEL}^{\text {var }}$ ] om. 757689 AMsted $^{\text {ed }}$ PEL $^{\mathrm{AB}}$ probatus est sed quem ] est probabilis sed quem 77 dominus commendat $\mathrm{SPE}^{\mathrm{var}}$ ] deus commendat $5^{1} 5^{8} 61 \mathrm{AMst}^{\mathrm{var}} \mathrm{PEL}^{\mathrm{var}}$ SPE ${ }^{\text {ed }}$, deum commendat 75

11:1 Present: 5154586164757677788889 AMst PEL CAr utinam ] + si 54 sustineretis ] pateremini $6177^{*}$, portaretis 7576 , patieremini $77^{\mathrm{C}}$, sustineritis 89

modicum ] paululum 61, pusillum 7576 AMst, paululum uel pusillum 77 quid ] qui $54^{*}$, om. 616475767789 AMst PEL ${ }^{\text {var }} \mathrm{CAr}$ insipientiae meae 51545888 ] inprudentiam meam 6177 AMsted $^{\text {ed }}$ PEL ${ }^{\text {var }}$, insipientiam meam 757689 CAr, prudentiam meam AMst ${ }^{\text {var }}$ sed et ] sed $88 * 89$ AMst $^{\text {var }}$ PEL $^{\text {var }}$, abs. CAr subportate me 5875767888 ] sustinete me 64 , subportate uel paterimini me 77 , sufferte me 89, patimini me AMst, abs. CAr

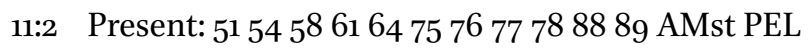
aemulor 5154586178 ] aemulans 7576 89, aemulor uel zelo 77 , zelans AMst 
enim uos $\langle 51\rangle]$ enim sum uos $\mathrm{AMst}^{\mathrm{R}}$

dei AMst ${ }^{\mathrm{var}}$ ] om. 64, deo $757689 \mathrm{AMst}^{\mathrm{ed}} \mathrm{PEL}^{\mathrm{var}}$

aemulatione 515458617888 ] aemulationem 64 , aemulor 757689 , zelo 77 $\mathrm{AMst}^{\mathrm{var}}$, zelor AMsted; + nam AMst ${ }^{\mathrm{R}}$

despondi 54617888 ] dei aptaui 64 , statui 757689 , desponsaui uel spopondi 77, paraui AMst ${ }^{\text {ed }}$, parui $\mathrm{AMst}^{\mathrm{A} * \mathrm{D} * \mathrm{R}}$

enim uos uni uiro uirginem

castam exhibere $\langle 64\rangle 88$ ] exhibere castam 54 , castam adsignare 61 AMst

$\mathrm{PEL}^{\mathrm{B}}$, castam adsignare uel exhibere 77

christo ] deo PEL var

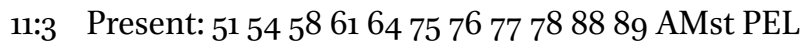

timeo autem ] timeo enim $6189 \mathrm{AMst}^{\mathrm{var}} \mathrm{PEL}^{\mathrm{var}}$

ne ] ṇọn $54^{*}$, ṇe $54^{\mathrm{C}}$; + forte $777^{8}$

sicut serpens

euam seduxit $\left.7588^{*}\right]$ seduxit euam $5^{8} 77$, fefellit $64^{*}$, euam fefellit $64^{\mathrm{C}}$; + de

$7576,+$ in 77

astutia sua 54 ] uersutia sua 64

ita ] tam 51, om. 647576 77, sic 89 AMst PEL ${ }^{\mathrm{B}}$

corrumpantur sensus uestri $\left(88^{*}\right)$ ] corrumpantur et sensus uestri 61 , cor-

rumpantur mentes uestrae 64, corrumpatur sensus uestri 7576 89, corrumpentur sensus uestri $77^{*}$, sensus uestri corrumpantur AMsted, sensus nostri corrumpantur AMstvar, uestri sensus corrumpantur PEL ${ }^{\mathrm{B}}$

et excidant ] ut excidant 51, et excedant 54, om. 6475767789 AMst, et excitant PEL var

a simplicitate 78 ] a simplicitate et castitate 616477 PEL, a castitate et simplicitate 7576 , a castitate $7^{\mathrm{gl}} \mathrm{AMst}$, om. 89 ; + dei AMst

quae est 5154586188 ] qui sunt 89

in christo ] in christo iesu $5^{1} 5^{8} 6164 \mathrm{AMst}^{\mathrm{PEL}}{ }^{\mathrm{B}}$, in christo domini 54 , in christo iesu domino nostro 89

11:4 Present: 51545861647576777888 89 AMst PEL

nam si ] si autem 64 , si quidem enim 77 , nam $P E L^{\text {var }}$

is qui uenit (54) ] qui uenit 6477 , his qui ueniunt $P E L^{B} ;+$ ad uos $P E L^{B}$

alium christum ] alium iesum 647576

praedicat 515458617788 ] praedicant $P E L^{B}$

quem non $\left(7^{*}\right)$ ] quem nos non $61 \mathrm{PEL}^{\mathrm{B}}$

praedicauimus $51545878 \mathrm{AMst}^{\mathrm{var}}$ ] praedicamus $6177 \mathrm{AMst}^{\mathrm{ed}}$

aut ] an AMstvar

alium spiritum ] spiritum alium 757677 , spiritum alterum 89 
accipitis $5489^{c} \mathrm{AMst}^{\mathrm{R}}$ ] accepistis ${ }_{51} 61647576778889^{*} \mathrm{AMst}^{\mathrm{ed}} \mathrm{PEL}^{\mathrm{B}}$ quem non ] om. AMstrar

accepistis 61 ] excepistis 77, om. AMst ${ }^{\mathrm{var}}$, accepimus PEL ${ }^{\mathrm{B}}$, acceperitis PEL ${ }^{\mathrm{var}}$ aut aliud euangelium $545^{8} 61$ ] aut euangelium aliud $757^{6} 77$, aut alium euangelium PEL ${ }^{\text {var }}$ + accepistis 77

quod non ] quem non 6489 , quam non AMstvar, om. $\mathrm{PEL}^{\mathrm{B}}$

recepistis ] accepistis $61 \mathrm{PEL}^{\mathrm{var}}$, suscepistis 647576 89, recepistis uel excepistis $77, \mathrm{om} . \mathrm{PEL}^{\mathrm{B}}$

recte ] bene 75767789

pateremini $(54)$ ] patimini 64 ; + uel patiremini 77

11:5 Present: 51545861647576777888 89 AMst PEL

existimo 64 ] exaestimo $89^{*}$, aestimo AMstrar

enim nihil me ${ }_{57} 54$ ] enim me nihil 586164757689 , me enim in nullo 77

$\mathrm{AMst}^{\text {var }}$, enim me in nullo $\mathrm{AMst}^{\text {ed }} \mathrm{PEL}^{\mathrm{B}}$, nihil me $\mathrm{PEL}^{\text {var }}$

minus $\left(88^{*}\right)$ ] inferiorem $77 \mathrm{AMst}^{\text {ed }} \mathrm{PEL}^{\mathrm{B}}$, infirmiorem $\mathrm{AMst}^{\mathrm{var}}$

fecisse $\langle 64\rangle$ ] esse 77 , fuisse $\mathrm{AMst}^{\mathrm{ed}} \mathrm{PEL}^{\mathrm{B}}$, om. $\mathrm{AMst}^{\mathrm{A}}$

\pm in uobis $5864757688^{\mathrm{C}} 89 \mathrm{PEL}^{\mathrm{var}}$, uobis PEL ${ }^{\mathrm{var}}$

magnis apostolis $78 \mathrm{PEL}^{\mathrm{var}}$ ] a magnis apostolis $51546 \mathrm{1}^{\text {alt }} 5888 \mathrm{PEL}{ }^{\mathrm{AB}}$, his qui supra modum sunt apostoli 61 , ab his qui ualde sunt apostoli $64 \mathrm{AMst}^{\mathrm{ed}}$, ab aliis apostolis 757689 , qui supra modum sunt apostolis 77 , supra modum apostolis $78^{\mathrm{gl}}$, iis qui ualde sunt apostoli AMst ${ }^{\mathrm{R}}$; + qui ante me fuerunt $5^{8}$

11:6 Present: 51545861647576777888 89 AMst PEL

et si ] nam et si $5^{1} 545^{8} \mathrm{AMst}^{\mathrm{R}}$, si autem et $64,\left[5^{-8}\right]$ et si $88^{*}$, sed et si AMsted

inperitus 51545861767888 ] idiota 77 , inpeditus 89

sermone (64) ] sum sermone $51545^{8} 61757688$ (89) PEL ${ }^{\mathrm{B}}$, sermone sum AMst

sed non ] non tamen $78 \mathrm{AMst}^{\mathrm{R}}$, non $\mathrm{AMst}^{\mathrm{var}}$

scientia 54 ] scientiam 89; + sed 64757677

in omnibus autem ] in omnibus $64 \mathrm{PEL}^{\mathrm{B}}$, in omni 7576

manifestatus sum ] manifestatus $54^{*} 7576$, manifestus sum 588889 AMst PEL $^{\text {var, }}$, manifestati facti 64 , manifestantes uel manifestatus sum 77 uobis ] in uobis $61647789 \mathrm{AMst}^{\mathrm{var}} \mathrm{PEL}^{\mathrm{B}}$, in omnes uos 7576

11:7 Present: 51545861647576777888 89 AMst PEL aut numquid 547778 ] an numquid 64, an 7576 89, aut numquam PEL var peccatum feci ] ad peccatum feci PEL var me ipsum ] me ipseum $54^{*}$, me ipse $\mathrm{PEL}^{\mathrm{B}}$ 
humilians ut uos ] humiliando ut uos 64 exaltemini ] exultemini 61, exaltaremini 64 quoniam gratis ] quod gratis 757689 euangelium dei 545861 ] dei euangelium 757677 89, euangelium PEL ${ }^{\text {var }}$ euangelizaui uobis 545864 ] praedicaui uobis $6777 \mathrm{AMst}^{\text {ed }}$, praedicaui in uobis $\mathrm{AMst}^{\mathrm{R}}$

11:8 Present: 51545861647576777888 89 AMst PEL

alias ecclesias 6178

expoliaui $616478 \mathrm{AMst}^{\mathrm{var}}$ ] grauaui 757689 , spoliaui $77 \mathrm{AMst}^{\mathrm{ed}}$

accipiens stipendium ] accipiens ab eis stipendium 64 , acceptis stipendiis

$757^{6} 89^{\mathrm{C}}$, accepistis stipendiis $89^{*}$, accepto stipendio AMst, accipiens PEL $^{\text {var }}$

ad ministerium uestrum ] ad uestram ministrationem 647576 , ad uestrum ministerium 77 , ad ministrationem uestram 89 , in ministerium uestrum $\mathrm{PEL}^{\mathrm{A}}$, ad ministerium stipendium uestrum PEL ${ }^{\text {var }}$

11:9 Present: 51545861647576777888 89 AMst PEL

et cum ] cum PEL var

essem apud uos $51545^{8} 75$ ] apud uos essem 61 AMst PEL $^{\mathrm{B}}$, apud uos fuissem

64

et egerem ] et egens 7576 , et egerim $\mathrm{AMst}^{\mathrm{A} * \mathrm{D} *}$

nulli onerosus 54 ] nulli grauis $61 \mathrm{AMst}^{\mathrm{ed}} \mathrm{PEL}^{\mathrm{B}}$, nemi[...] 64, nemini honore $75^{*}$, nemini oneri $75^{\mathrm{C}} 76$, non grauis 77 , nulli onere 89 , nullis grauis $\mathrm{AMst}^{\mathrm{var}}$, nihil grauis PEL ${ }^{\mathrm{var}}$

fui nam ] lac. 64, fui ulli nam 77 , fuit nam AMst ${ }^{\mathrm{var}}$

quod mihi deerat 58 ] id quod mihi deerat $61 \mathrm{PEL}^{\mathrm{B}}$, lac. 64 , quod deerat mihi

757689 , id quod deerat mihi 77 , indigentiam meam AMst

suppleuerunt fratres ] adimpleuerunt fratres $6{ }_{77} \mathrm{PEL}^{\mathrm{B}}$, lac. 64, fratres suppleuerunt 89

qui uenerunt 61 ] lac. 64, uenientes 77 , qui uenerant $P E L^{v a r}$

a macedonia $54^{c} 617576(89)$ ] ad macedonia $54^{*}$, lac. 64 , de macedonia PEL var

et in ] lac. 64, et $7576 \mathrm{PEL}^{\mathrm{B}}$, in $\mathrm{AMst}^{\mathrm{var}}$

omnibus ] lac. 64 , om. $\mathrm{PEL}^{\mathrm{B}}$; + uobis me 58 , + me PEL var

sine onere 61 ] om. $64 \mathrm{PEL}^{\mathrm{var}}$, sine honore $75^{*} 88 \mathrm{AMst}^{\mathrm{var}} \mathrm{PEL}^{\mathrm{var}}$; + uestro 61 AMst PEL ${ }^{\mathrm{B}}$

me uobis ] uobis me $5_{11} 77$, om. $5^{8} \mathrm{PEL}^{\text {var }}$, lac. 64 , me ipsum AMst, me PEL ${ }^{\mathrm{B}}$ seruaui et seruabo ] lac. 64 , seruam et seruo $\mathrm{PEL}^{\mathrm{B}}$ 


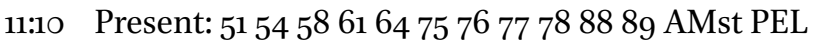

est ueritas christi in me ] est enim ueritas christi in me 61 quoniam $\mathrm{AMst}^{\mathrm{R}}$ ] quia 64 , quod $7576 \mathrm{AMst}^{\mathrm{ed}}$, eo quod 89 haec gloria 515488 ] haec gloriatio 586 6 $\mathrm{PEL}^{\mathrm{B}}$, gloria haec $64757689 \mathrm{AMst}$, gloriatio haec 77 ; + mea 58

non infringetur 61 ] non fringetur 58 , non frangetur uel non obstrueretur uel obstruetur $\left(77^{*}\right)^{\mathrm{C}}$, non infringitur PEL ${ }^{\mathrm{A}}$

in me ] et AMst ${ }^{\text {var }}$

in regionibus achaiae 5154586188 ] in finibus achaiae 75767789 , in partibus achaiae AMst ${ }^{\text {var }}$

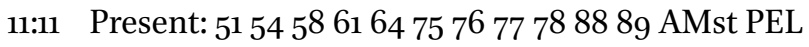

quare quia ] quare qui AMst ${ }^{\mathrm{var}} \mathrm{PEL} \mathrm{Lar}^{\mathrm{var}}$

non diligo uos deus scit ] uos non diligo deus scit $67\langle 64\rangle \mathrm{PEL}^{\mathrm{B}}$, non uos diligo uos deus scit 77

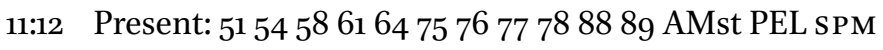

quod autem facio ] quod facio PEL ${ }^{\text {var }}$ + faciam 61

et ] om. $58^{*} 88^{*} \mathrm{AMst}^{\mathrm{var}}$

faciam ] facturus sum $616477 \mathrm{AMst}_{\mathrm{PEL}}^{\mathrm{B}} \mathrm{SPM}^{\mathrm{var}}$

ut amputem occasionem $616475\langle 76\rangle 7789$

eorum qui uolunt ] eorum 54 , uolentium 77, om. $88^{*}$, eis qui uolunt $\mathrm{AMst}^{\mathrm{R}}$, meam qui uolunt AMstrar

occasionem ut 6164757689 ] ut $5488^{*}$; + in eo 64 AMst SPM $^{\text {var }}$

in quo $\mathrm{AMst}^{\mathrm{A}}$ ] et in quo 54, quod $64 \mathrm{AMst}^{\mathrm{ed}} \mathrm{SPM}^{\mathrm{var}}$, de quo $\mathrm{AMst}^{\mathrm{R}}$, quo AMst $^{\text {var }}$

gloriantur

inueniantur ] tales inueniantur $51586188^{\mathrm{C}} \mathrm{PEL}^{\mathrm{B}}$, inuenientur $54^{*}$, inueniantur tales PEL var

sicut et nos $61^{\text {alt }}$ ] sicut et uos 61 , sicut nos $88^{*}$

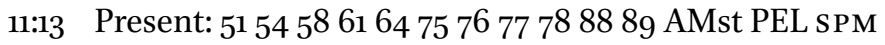

nam eiusmodi ] nam huiusmodi 64 SPM, nam tales uel eiusmodi uel huiusmodi 77 , huiusmodi enim AMst; + sunt PEL var

pseudoapostoli $61\langle 64\rangle(77)]+$ sunt 586175767789 AMst $^{\text {ed }} \mathrm{PEL}^{\mathrm{AB}}$

operarii 5461$]+$ sunt AMst ${ }^{\text {var }}$

subdoli ] dolosi 6475767789 sPM $^{\text {var }}$, subdol[1-2] 88*, subdolo $88^{\mathrm{C}}$, nequitiae dolosi SPM ${ }^{\text {ed }}$

transfigurantes $\left.(54)\langle 64\rangle\left(\mathrm{PEL}^{\mathrm{A} *}\right)^{\mathrm{C}}\right]$ transfigurant $\mathrm{PEL}^{\mathrm{B}}$; + uel transfigurati 77 se in apostolos christi ] se in apostolis christi 89, in apostolos AMst ${ }^{\text {var }}$ 


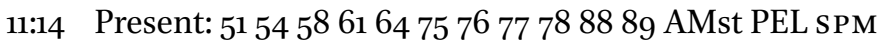

et non (54) ] neque $61 \mathrm{PEL}^{\mathrm{B}}$, nec 7576 89, neque uel et non 77 , nec utique AMst, sed non SPM

mirum ] mirandum 7576 , mirum est $P E L^{\text {var }}$

ipse enim SPM ${ }^{\text {var }}$ ] cum ipse $61 \mathrm{PEL}^{\mathrm{B}} \mathrm{SPM}^{\mathrm{ed}}$, cum ipse enim $77^{\mathrm{C}}$

satanas $515^{8}$

transfigurat se $\left(54^{*}\right) \mathrm{SPM}^{\mathrm{var}}$ ] transfiguratur AMst, transfiguret se $\mathrm{PEL}^{\mathrm{B}}$ $\mathrm{SPM}^{\mathrm{ed}}$

in $\mathrm{SPM}^{\mathrm{var}}$ ] ut 61, sicut 7576 89, in uel ut 77 , uelut AMst PEL var SPM $^{\text {ed }}$

angelum ] gloriam $54^{*}$, angelus 617589 AMst, angeli PEL ${ }^{\text {var }}$

lucis ] luminis AMst

11:15 Present: 51545861647576777888 89 AMst PEL SPM

non ] + magnum $77 \mathrm{AMst}^{\mathrm{ed}}$

est ergo ] est $61 \mathrm{SPM}^{\mathrm{ed}}$, ergo $64 \mathrm{AMst}^{\mathrm{var}}$, om. 757689 , ergo si et $77 \mathrm{AMst}^{\mathrm{ed}}$, est $\mathrm{e}[3]$ 88*, ergo est PELvar, est enim SPM ${ }^{\text {var }}$

magnum si ministri eius $\left.\langle 76\rangle \mathrm{AMst}^{\mathrm{var}}\right]$ ministri eius $77 \mathrm{AMst}^{\mathrm{ed}}$ transfigurentur $\mathrm{SPM}^{\mathrm{var}}$ ] transfigurantur $61757689 \mathrm{AMst}^{\mathrm{PEL}}{ }^{\mathrm{var}} \mathrm{SPM}^{\mathrm{ed}}$, transfigurụntur 88

uelut $58 \mathrm{AMst}^{\mathrm{var}}$ ] sicut $64757689 \mathrm{AMst}^{\mathrm{ed}}$, ut SPM ${ }^{\mathrm{var}}$

ministri iustitiae quorum finis $51545^{8}$

erit secundum ] secundum 64, est secundum 757689 sPM

opera ipsorum $\left.\mathrm{SPM}^{\mathrm{var}}\right]$ ipsorum opera 51 , operationem ipsorum 6489 , opera eorum AMst $\mathrm{SPM}^{\text {ed }}$, eorum $\mathrm{SPM}^{\mathrm{var}}$

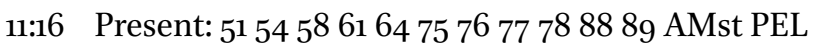

iterum $\langle 88\rangle$ ] et iterum PEL ${ }^{\text {var }}$

dico ne quis ] dico uobis ne quis 61 , uindico ne quis AMstrar

me putet ] me existimet $616475767789^{\mathrm{C}} \mathrm{AMst}^{\mathrm{ed}} \mathrm{PEL}^{\text {var }}$, me exaestimet

$89^{*}$, existimet me AMstvar

insipientem ] + esse 515458647778

alioquin ] si autem 77

uelut insipientem 58 ] uelut sapientem 89

accipite me ] suscipite me 64757689 , suscipiat accipiat me 77

ut et ego ] ut ego $64757688^{*}$ PEL $^{\text {var }}$

modicum ] pusillum 757689

quid $54 \mathrm{AMst}^{\mathrm{R}}$ ] aliquid 61 $\mathrm{AMst}^{\mathrm{ed}}$, om. PEL ${ }^{\mathrm{var}}$

glorier (78) ] gloriarer AMst ${ }^{\mathrm{var}}$, gloriar PEL ${ }^{\mathrm{var}}$ 


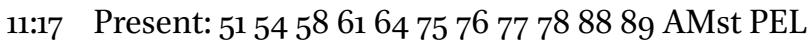

quod loquor non ] quod PELA*

loquor secundum $\langle 64\rangle$ ] secundum 7778

dominum PEL ${ }^{\text {var }}$ ] deum $545861647888^{\mathrm{C}}$ AMst PEL ${ }^{\mathrm{AB}}$; + loquor 7778

sed quasi ] sed ut $6177 \mathrm{PEL}^{\mathrm{B}}$, sed quia si AMstvar, sed PEL var

in ] om. $77 \mathrm{AMst}^{\mathrm{var}} \mathrm{PEL}^{\mathrm{A} *}$

insipientia ] insipientiam $5489 \mathrm{AMst}^{\mathrm{var}} \mathrm{PEL}^{\mathrm{B}}$, stultitia 64

in hac ] om. 64 PEL var

substantia 54 ] om. 64 , substantiae $P E L^{A *}$

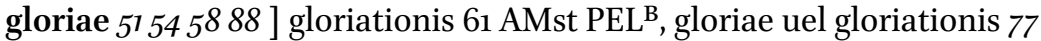

11:18 Present: 51545861647576777888 89 AMst PEL

quoniam ] quia $61757689 \mathrm{AMst}^{\mathrm{ed}} \mathrm{PEL}^{\mathrm{B}}$, om. 64 , quando quidem $\mathrm{AMst}^{\mathrm{R}}$; + uel quia 77

multi gloriantur ] multis gloriantur 75 , gloriantur multi AMstvar

secundum carnem et ego ] secundum carnem et 89, et ego secundum carnem PEL ${ }^{B}$

gloriabor ] glorior 64

11:19 Present: 5154586164757677788488 89 AMst PEL

libenter enim ] fratres libenter 84

suffertis insipientes $\left(78^{*}\right)$ ] sustinetis insipientes AMst

cum sitis ipsi sapientes ] cum sitis sapientes $647789 \mathrm{AMst}^{\mathrm{A}}$, cum sapientes sitis $7576, a b s .84$

11:2O Present: 51545861647576777888 89 AMst PEL

sustinetis enim ] toleratis enim 64 , suffertis enim 757689 ; + ipsi 61 si quis uos in seruitutem ] si quis in seruitutem uos 61

redigit ] redegit 51 , redegerit $61 \mathrm{PEL}^{\text {var(ed) }}$, redigerit $\mathrm{PEL}^{\mathrm{A}}$

si quis deuorat si quis 64

accipit ] accepit 89 PEL ${ }^{\mathrm{var}}$

si quis extollitur ] si quis extolletur PEL var

si quis in faciem uos AMstrar $]$ si quis uos in faciem $89 \mathrm{AMst}^{\mathrm{ed}}$

caedit 51545861647677788889

11:21 Present: 5154586164757677788889 AMst PEL

secundum ignobilitatem dico $(75)] \mathrm{om}$. $\mathrm{AMst}^{\mathrm{AD} *}$

quasi ] quasi quia 77 , quia si $\mathrm{AMst}^{\mathrm{var}}$

nos infirmi fuerimus ] nos infirmati sumus 64 , infirmi fuerimus nos 77 , nos

infirmi fuemus 88 
\pm in hac parte $51545^{8} 617576 \mathrm{AMst}^{\mathrm{PEL}} \mathrm{L}^{\mathrm{B}}$, in hoc parte $88^{*}$

in quo ] + autem 647778

quis audet ] quis au[det $] 64$, quis audit $\mathrm{PEL}^{\mathrm{var}}$; + audeo et ego 77

in ] lac. 64 , om. 89

insipientia dico $54 \mathrm{AMst}^{\mathrm{var}}$ ] lac. 64, insipientiam dico $757689 \mathrm{AMst}^{\mathrm{ed}}$ PEL $^{B}$

audeo et ego ] audeat et ego $54^{*}$, lac. 64, om. 77 AMstrar

11:22 Present: 515458617576777888 89 AMst PEL

hebraei sunt et ego 51545861757677788889

israhelitae 5158617788 AMst ] israhelita 54

sunt et ego semen

abrahae 515458617788 ] abraam 75 , abraham 7689

sunt et ego

11:23 Present: 515458617576777888 89 AMst PEL SPE

ministri christi sunt $\mathrm{AMst}^{\mathrm{var}}$ ] ministri sunt christi $77 \mathrm{AMst}^{\mathrm{ed}}$

\pm et ego $515458617888^{\mathrm{C}} 89 \mathrm{AMst}^{\mathrm{R}} \mathrm{PEL}^{\mathrm{B}} \mathrm{SPE}^{\mathrm{var}}$

minus ] ut minus $5^{1} 545^{8} 617576788889$ PEL SPE, ut 77, uelut AMst sapiens dico ] insipiens dico 77 AMst

plus AMst ${ }^{\mathrm{var}}$ ] magis $6177 \mathrm{AMst}^{\mathrm{ed}} \mathrm{PEL}^{\mathrm{B}}$, super 7576 , plurimum $\mathrm{AMst}^{\mathrm{R}}$

ego in laboribus ] et ego in laboribus PEL var

plurimis ] abundantius 77, pluribus 89; + magis ego in laboribus PEL ${ }^{\text {var }}$

in carceribus abundantius in plagis 5154586188 ] in plagis abundantius in carceribus 77

supra modum ] super modum PELA

in mortibus frequenter ] in mortibus saepius AMst

11:24 Present: 515458617576777888 89 AMst PEL SPE

a iudaeis quinquies 51545861 (77) 7888 ] ab iudaeis quinquies 77

quadragenas $6 \mathrm{AMst}^{\mathrm{var}} \mathrm{SPE}^{\mathrm{var}}$ ] quadraginta $54^{*} 757^{2} \mathrm{AMst}^{\mathrm{ed}} \mathrm{PEL}^{\mathrm{var}} \mathrm{SPE}^{\mathrm{ed}}$ una minus accepi $\left.\left(78^{*}\right)\right]$ unam minus accepi 61

11:25 Present: 515458617576777888 89 AMst PEL SPE

ter uirgis caesus sum semel lapidatus sum 5154586177788889

ter naufragium feci ] ter naufragatus sum 77

nocte et die ] nocte ac die $5_{1} \mathrm{AMst}^{\mathrm{var}} \mathrm{PEL}^{\mathrm{var}}$, noctem et diem $757^{6}$

in profundo ] in profundum $88^{*} 89 \mathrm{AMst}^{\mathrm{var}} \mathrm{PEL}^{\mathrm{B}} \mathrm{SPE}^{\mathrm{var}}$

maris fui ] maris feci 7576 , fui $77^{*}$ 
11:26 Present: $51545^{8} 617576777^{8} 8889$ AMst PEL SPE

in ] om. $7576 \mathrm{PEL}^{\mathrm{A}}$

itineribus ] itinere $\mathrm{AMst}^{\mathrm{var}}$

saepe ${ }_{51} 5^{8} 617888 \mathrm{AMst}^{\mathrm{var}}$ ] spe 54 , multis 7576 , crebrius AMsted

periculis fluminum periculis latronum periculis $(61)$

ex genere periculis ex gentibus ] ex gentibus periculis ex genere 61

periculis in ciuitate

periculis in ] in $5^{8}$

solitudine ] solitudinem 58 , deserto $61757689 \mathrm{AMst} \mathrm{PEL}^{\mathrm{B}}$, deserto in eremo uel solitudine 77

periculis in mari ] periculis in mare $\mathrm{SPE}^{\mathrm{var}}$

periculis in falsis fratribus AMst ${ }^{\mathrm{var}}$ ] periculis in fictis fratribus AMsted

11:27 Present: $51545^{8} 617576777888$ 89 AMst PEL SPE

in ] om. 757677

labore et aerumna 515458777888 ] laboribus in miseriis $61 \mathrm{PEL}^{\mathrm{B}}$, labore et molestia AMst; + et PEL var

in uigiliis multis ] in uigiliis saepe $77 \mathrm{AMst}^{\mathrm{R}}$

in fame et siti ] in fame in siti 61

in ieiuniis multis ] in ieiuniis saepe uel multis (77)

in frigore et nuditate

11:28 Present: 51545861757677788889 AMst PEL SPE

praeter illa quae $51545^{8} 67_{7} 678$ ] praeter ea quae 77 , praeter quae $S P E^{\text {var }}$ extrinsecus ] intrinsecus PEL ${ }^{\text {var }}$

sunt ] om. 78

instantia mea cotidiana 54586178 AMst ] concursus in me cotidianus 7576

89

sollicitudo 5861 ] sollitudo $75^{*}$, sollicitudine $\mathrm{SPE}^{\mathrm{var}}$

omnium ecclesiarum 61 ] + dei 61

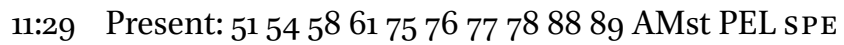

quis infirmatur ] quis enim infirmatur $\mathrm{AMst}^{\mathrm{var}}$

et non ] et ego non $5^{1} 545^{8617888} 89$ PEL SPE $^{\text {ed }}$, et non ego AMst SPE ${ }^{\text {var }}$ infirmor ] [2] firmor $88^{*}$, infirmar $89^{*}$, infirmo $\mathrm{PEL}^{\mathrm{B}}$

quis scandalizatur 58 ] qui scandalizatur $89 \mathrm{PEL}^{\mathrm{var}}$, qui scandalizetur $\mathrm{SPE}^{\mathrm{var}}$ et ego non uror $\mathrm{AMst}^{\mathrm{var}} \mathrm{SPE}^{\mathrm{var}}$ ] et non ego uror $757^{6} 77 \mathrm{AMst}^{\mathrm{ed}} \mathrm{SPE}^{\mathrm{ed}}$

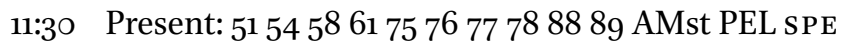
si gloriari oportet ] + in iis $\mathrm{AMst}^{\mathrm{R}}$, + in his $\mathrm{AMst}^{\mathrm{var}}$ 
quae 515458617888 ] + sunt 6177

infirmitatis ] infirmitates 89

meae 515476777888 ] me 58 ; + in his 61

sunt gloriabor ] gloriabor 617789 *

11:31 Present: $51545^{8} 61757677788889$ AMst PEL

deus et pater ] deus israhel et pater 7576 , deus pater AMst ${ }^{\text {var }}$

domini iesu ] domini nostri iesu christi 51 (54) 58617576788889 AMst PEL scit qui est ] scit 7576

benedictus in saecula $51545877>8$

quod non mentior $\mathrm{AMst}^{\mathrm{R}}$ ] quia non mentior $757689 \mathrm{AMst}^{\mathrm{ed}}$, quod uel quia non mentior 77

11:32 Present: 515458617576777888 89 AMst PEL

damasci ] in damasco uel damasci 77

praepositus gentis 5154587888 ] princeps gentis 6177 AMst, gentis princeps $7^{\mathrm{gl}}$; + princeps $\mathrm{PEL}^{\mathrm{B}}$

aretae $51545^{8} 7^{c}$ ] aratae $6178^{*} 89$ AMst $\mathrm{PEL}^{\mathrm{A} *}$, ariet[2] $61^{\text {alt }}$

regis custodiebat ] reges custodiebant $P E L^{\text {var }}$

ciuitatem damascenorum (61)

ut me conprehenderet 5178 ] ut me caperet 7576 , uolens conprehendere me 77

11:33 Present: 515458617576777888 89 AMst PEL

et per fenestram 6177 ] ut per fenestram 75

in sporta ] in sportam $757689 \mathrm{AMst}^{\mathrm{var}} \mathrm{PEL}^{\mathrm{B}}$

dimissus sum $\left(78^{*}\right)$ ] missus sum 757689 , submissum 77 , demissus sum $88^{\mathrm{C}}$

$\mathrm{AMst}^{\mathrm{var}}$, sum missus non $\mathrm{PEL}^{\mathrm{B}}$

per murum et $\left.(58) \mathrm{PEL}^{\text {var }}\right]+\operatorname{sic} 51546188 \mathrm{PEL}^{\mathrm{AB}}$

effugi manus eius 58

12:1 Present: 515458617576777888 89 AMst PEL CAr si AMstvar ${ }^{\text {va }}$ om 75767789 AMst $^{\text {ed }}$ PEL $^{\mathrm{B}}$, abs. CAr gloriari oportet ] abs. CAr; + sed AMst ${ }^{\text {ed }}$, + et $\mathrm{PEL}^{\mathrm{B}}$ non expedit $\mathrm{AMst}^{\mathrm{var}}$ ] non opus est $\mathrm{AMst}^{\mathrm{ed}}$, abs. CAr

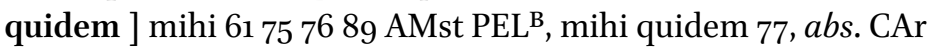
ueniam autem ad ] ueniam ei ad 7576 , ueniam ad PEL var uisiones et reuelationes $617588^{*}$ ] uisiones PEL var domini ] christi 77 
12:2 Present: 515458617576777888 89 AMst PEL FOR

scio hominem in christo ante annos quattuordecim 5178 ] abs. FOR siue in corpore nescio ] siue in corpore $515861 \mathrm{PEL}^{\mathrm{A}}, a b s$. FOR siue extra corpus (88) ] siue extra corpore $617576 \mathrm{AMst}^{\mathrm{var}}$, abs. FoR; + uel corpore 77

nescio deus scit raptum ] nescio deus scit raptus 89 , abs. FOR eiusmodi PEL ${ }^{\text {var }}$ ] huiusmodi 515458617778 AMst $^{\text {ed }}$ PEL $^{\mathrm{AB}}$, om. AMst $^{\mathrm{A}}$, abs. FOR

usque ad (89) ] usque in FOR

tertium caelum 5154587788 ] tertium $89^{*}$

12:3 Present: 51545861757677788889 AMst PEL

et scio

huiusmodi ] eiusmodi 7576 89, talem uel huiusmodi 77, talem AMst

hominem siue in corpore ] + nescio AMst ${ }^{\mathrm{var}}$

siue extra corpus $(77)]+$ uel corpore 77

nescio deus scit (88)

12:4 Present: 51545861757677788889 AMst PEL FOR quoniam $\mathrm{AMst}^{\mathrm{D}}$ ] quia $757689 \mathrm{AMst}^{\mathrm{ed}}$, om. AMst $^{\mathrm{var}} \mathrm{FOR}$ raptus est in paradisum ${ }_{51}$ ] raptus est in paradiso $89 \mathrm{PEL}^{\text {var, }}$, in paradisum raptus FOR

et audiuit ] et audiui $8889 \mathrm{PEL}^{\mathrm{var}}\langle\mathrm{FOR}\rangle$

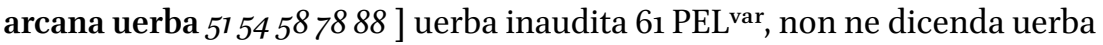

7576 , inaudita uerba 77 , non edicenda uerba 89 , inenarrabilia $\mathrm{FOR}^{\mathrm{ed}}$, iner-

rabilia uerba $\mathrm{FOR}^{\mathrm{var}}$, enarrabilia $\mathrm{FOR}^{\mathrm{var}}$ quae non $51545861777888(89)$ ] abs. FOR

licet ] licent 58 , licebat $\mathrm{AMst}^{\mathrm{AD} *} \mathrm{PEL}^{\text {var }}$, abs. FOR

homini loqui ] hominibus loqui $61 \mathrm{PEL}^{\mathrm{A}}$, homin[2] loqui $88^{*}$, abs. FOR

12:5 Present: 51545861757677788889 AMst PEL

pro eiusmodi $\left(7^{*}\right)$ ] pro huiusmodi $51545^{8} 77$ AMst

gloriabor pro me autem

nihil gloriabor $51545^{8}$ ] non gloriabor 77 AMst, gloriabor nihil 89*, nihil PEL $^{\mathrm{B}}$

nisi in ] nisi 75 AMstvar $^{\text {var }}$

infirmitatibus meis ] infirmitatibus 7576

12:6 Present: 51545861757677788889 AMst PEL

nam et si ] nam si 51, si enim 61757677 89, et si AMst ${ }^{\mathrm{R}}$, nam et $\mathrm{PEL}^{\mathrm{A}}{ }^{*}$ 
uoluero gloriari ] uoluero gloriabor $54^{*}$

non ero ] non sum PEL var

insipiens ] stultus AMst PEL ${ }^{B}$

ueritatem enim ] ueritatem 61, nam ueritatem 757689 AMst, iam enim ueritatem PEL ${ }^{\mathrm{B}}$

dicam AMstrar ] dico $6178 \mathrm{AMst}^{\mathrm{ed}}$, dicam uel dico 77 ; + dico in christo 58

parco autem ne quis in ] parco autem ne quis $515458617888 \mathrm{AMst}$ PEL

me existimet ] existimet me 58 , me exaestimet $89^{*} P E L^{A}$

supra ] super AMst ${ }^{\mathrm{var}}$

id quod 54 ] quod 77 , quam quod PEL ${ }^{\text {var }}$

uidet me $54 \mathrm{AMst}^{\mathrm{var}}$ ] uidet in me $51{ }^{8} \mathrm{AMst}^{\mathrm{ed}}$, uidet $61 \mathrm{PEL}^{\mathrm{B}}$, me uidet 75 76 89, uidit me $88^{*}$ PEL $^{\text {var }}$, uidet esse me AMst $^{\mathrm{R}}$

aut audit ] aut 51 , aut audet $88^{*}$, uel audit AMstrar

ex me ] aliquid ex me $5^{1} 5^{8} 757678$ AMst, quid ex me PEL ${ }^{B}$

12:7 Present: 515458617576777888 89 AMst PEL tes

et ne ] et 6175767789 , abs. TES

magnitudo ] in illa abundantia 61 , in sublimitate 7576 , abundantia 77 , sublimitate 89 AMst $^{\text {ed }}$, sublimitas AMst ${ }^{\mathrm{var}}$, abs. TES

reuelationum ] reuelation[ 1$]$ 89*, abs. TES; + propter quod ut 77

extollat me ] ne extollar 61757677 89, extollar AMst ${ }^{\text {ed }}$, extollet AMst ${ }^{\mathrm{tar}}$, abs. TES

datus est mihi (88) ] datus est enim mihi PELvar

stimulus carnis 5158 ] stimulus carni $77 \mathrm{TES}^{\mathrm{var}}$

meae 51545877 ] om. 7576 AMst

angelus satanae 545861778889$] \mathrm{om} .5^{1}$

ut me (78) ] qui me 61 AMst $^{\mathrm{A}}$ TES, ut PEL ${ }^{\text {var }}$

colaphizet 545861788889

\pm ut non extollar $61 \mathrm{PEL}^{\mathrm{B}} \mathrm{TES}^{\mathrm{ed}}$, ne extollar AMst ${ }^{\mathrm{ed}}$, ne extollatur $\mathrm{AMst}^{\mathrm{AD}}$, non extollor TES ${ }^{\text {var }}$, ut non extollat TES $S^{\text {var }}$

12:8 Present: 515458617576777888 89 AMst PEL tes

propter quod ] propter hoc 6175767789 AMst

ter dominum rogaui $\mathrm{AMst}^{\mathrm{var}}$ ] dominum ter rogaui $757689 \mathrm{TES}^{\mathrm{var}}$, dominum rogaui $\mathrm{AMst}^{\mathrm{ed}} \mathrm{TES}^{\mathrm{var}}$, ter rogaui dominum PEL ${ }^{\mathrm{var}}$, deum rogaui TES ${ }^{\text {var }}$

ut discederet a me $\left(77^{*}\right)$ ] ut descenderit a me AMst ${ }^{\mathrm{var}}$, ut discesserit a me TES var 
12:9 Present: 51545861757677788889 AMst PEL TES SPE

et dixit mihi $5^{8} \mathrm{AMst}^{\mathrm{var}}$ ] et ait mihi $\mathrm{AMst}^{\mathrm{ed}} \mathrm{TES}^{\mathrm{var}}$, abs. SPE; + dominus TES var

sufficit tibi ] sufficiat tibi TES ${ }^{\mathrm{var}}$, abs. SPE; + paule $5^{8}$

gratia mea ] gratia PEL ${ }^{\text {var }}$, gratiam meam TE ${ }^{\text {var }}$, abs. SPE

nam uirtus ] abs. SPE

in infirmitate ] infirmitate 88 , in infirmitatem 89, om. TE ${ }^{\text {var }}, a b s$. SPE

perficitur ] consummatur $\mathrm{AMst}^{\mathrm{R}}$, abs. SPE

libenter ] libentissime $75767789 \mathrm{AMst} \mathrm{PEL}^{\mathrm{B}}$, abs. TES

igitur ] itaque magis $6175767789 \mathrm{AMst}$, magis itaque $\mathrm{PEL}^{\mathrm{B}}$, abs. TES

gloriabor in infirmitatibus meis ut ] abs. TES

inhabitet 54 ] habitet 61, praetendat $\mathrm{AMst}^{\mathrm{R}}$, abs. TES

in me uirtus christi ] in me uirtus dei AMst ${ }^{\text {var }}$, abs. TES

12:1O Present: 51545861757677788889 AMst PEL SPE

propter quod ] quapropter AMsted, propterea $\mathrm{AMst}^{\mathrm{var}}$

placeo mihi 58 ] placeo 757677

in infirmitatibus ] om. $78^{*}$; + meis $586177 \mathrm{AMst}^{\mathrm{var}} \mathrm{PEL}^{\mathrm{var}}$

in contumeliis 61 ] om. $78^{*} \mathrm{AMst}^{\mathrm{var}}$

in necessitatibus $\mathrm{AMst}^{\mathrm{ADR}}$ ] om. AMst $^{\text {ed }} \mathrm{PEL}^{\mathrm{A}}$

in persecutionibus in angustiis $\left.\mathrm{AMst}^{\mathrm{ADR}}\right] \mathrm{om}$. $\mathrm{AMst}^{\mathrm{ed}}$, in angustiis in persecutionibus PEL ${ }^{\mathrm{B}}$

pro christo $\mathrm{AMst}^{\mathrm{ADR}}$ ] om. AMst $\mathrm{ed}^{\mathrm{d}}$

cum enim infirmor tunc ] cum enim infirmior tunc $\mathrm{SPE}^{\mathrm{var}}$

potens sum ] potentor uel potens sum 77 , potens sum factus PEL ${ }^{\text {var, }}$, potens factus sum PEL var

12:11 Present: 515458617576777888 89 AMst PEL

factus sum insipiens (54) ] insipiens factus sum $P E L^{B}$, factus insipiens

PEL ${ }^{\text {var }}$, insipiens PEL ${ }^{\text {var }}$ + gloriando 89

uos me coegistis ] uos me cogistis 6189

ego enim debui a uobis 88 ] ego enim habui a uobis $88^{\text {alt } \dagger}$

commendari 54 ] commendare 75 , emendari PEL ${ }^{\mathrm{var}}$

nihil enim $51545^{8}$ ] nam nihil $61 \mathrm{PEL}^{\mathrm{B}}$, non enim 77 , nihil 88

minus fui ] minus 51, minus feci $5^{8} 757^{6} 7789$ AMst PEL ${ }^{\text {var }}$

ab his 58 ] ab aliis 757689 , quam illi AMst

qui sunt ] qui ${ }_{51} 61757677 \mathrm{PEL}^{\mathrm{B}}$, om. 89 AMst

$\dagger 2$ Cor. 12:11. The gloss habui may go with debui or with fui later in the verse. 
supra modum ] su[2] a modum $54^{*}$, supra modum ualde $61 \mathrm{PEL}^{\mathrm{B}}$, super ualde 77, om. 89, ualde AMst, super modum PELA ; + sunt $61757677^{\mathrm{C}} \mathrm{PEL}^{\mathrm{B}}$ apostoli ] apostolis 7789

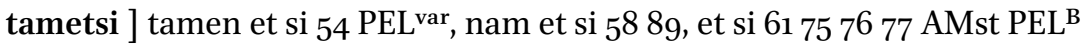
nihil sum $51545^{8}$

12:12 Present: 51545861757677788889 AMst PEL signa tamen $\mathrm{AMst}^{\mathrm{R}}$ ] tamen signa $617576 \mathrm{AMst}^{\mathrm{ed}}$, sed tamen uel quidem signa $77,{ }^{\dagger}$ sed signa $\mathrm{PEL}^{\mathrm{B}}$, signa uero $\mathrm{PEL}^{\text {var }}$ apostoli ] apostolatus $5^{1} 5^{861} 77\left\langle 88^{*}\right\rangle \mathrm{PEL}^{\mathrm{B}}$, om. AMst ${ }^{\mathrm{AD}}$; + mei $5^{1} 5^{861}$ facta sunt ] perfecta sunt $61 \mathrm{PEL}^{\mathrm{B}}$, sunt facta $78^{*}$ super uos PEL var ] inter uos $61757677 \mathrm{PEL}^{\mathrm{AB}}$, in uobis 89 AMst in omni ] + uirtute et $5^{8}$ patientia 5154768889 ] potentia $\mathrm{AMst}^{\text {var }} \mathrm{PEL}^{\mathrm{B}}$; + in 54 , + et $5^{8} 77$ signis et prodigiis et uirtutibus ] signis et prodigiis et uariis uirtutibus 61

12:13 Present: 51545861647576777888 89 AMst PEL quid est enim $\langle 76\rangle$ ] lac. 64 , quid enim est 77 ; + quod dicimus $5^{1}$ quod minus habuistis 54 ] lac. 64, quod minus habueritis $\mathrm{PEL}^{\mathrm{B}}$ prae ceteris ecclesiis $51545861777888 \mathrm{AMst}^{\mathrm{D}}$ ] lac. 64, quam ceterae ecclesiae 757689 , ceteris ecclesiis AMst ${ }^{\text {ed }}$, prae ceteris apostolis PEL var nisi quod ego ipse ] nisi quod ego 61, lac. 64, nisi quod ipse PEL var non grauaui uos donate mihi 5458 (78) 89 ] lac. 64 hanc iniuriam 54 ] [iniuriam is] tam 64, iniuriam hanc 757677

12:14 Present: $5^{1} 545^{8} 6164757677788889$ AMst PEL CAr SPE ecce tertio $5154 \mathrm{PEL}^{\text {var }}$ ] ecce tertium $6475767789 \mathrm{PEL}^{\mathrm{B}}$, om. $\mathrm{PEL}^{\mathrm{A}}$ hoc ] lac. 64, om. $89 \mathrm{AMst}^{\mathrm{R}} \mathrm{PEL}^{\mathrm{A}}$ paratus sum ] parati sumus $6189, \mathrm{p}[\ldots] 64$, om. $\mathrm{PEL}^{\mathrm{A}}$ uenire ad uos et non ] om. PEL ${ }^{\mathrm{A}}$ ero grauis uobis PEL var ] uos grauabo 61757689 AMst PEL ${ }^{B}$, grauabo 64, grauabo uos 77, om. PEL $^{\mathrm{A}}$, ero uobis grauis PEL var non enim quaero quae uestra sunt $51545861\langle 64\rangle 76777888$ ] abs. CAr sed uos $\langle 64\rangle]$ om. $\mathrm{PEL}^{\mathrm{B}}$, abs. CAr; + et non ero grauis uobis $58^{*}$ nec ] non 586177 , lac. 64, abs. CAr enim ] om. $\mathrm{PEL}^{\mathrm{B}}$, abs. CAr debent filii parentibus 7589 ] debent filii parentes $88^{*}$, abs. CAr

$\dagger{ }_{2}$ Cor. 12:12. In VL 77 , tamen may be a misreading of the Greek $\tau \alpha \mu \varepsilon v$ below. 
thesaurizare $545861\langle 64\rangle 7576$ ] thesaurizari 89, abs. CAr sed parentes filiis $\left.\left(54^{*}\right)\right]$ abs. CAr

12:15 Present: 51545861647576777888 89 AMst PEL SPE

ego autem $\langle 64\rangle$ ] ego uero $\mathrm{AMst}^{\text {ed }}$, ego uere $\mathrm{AMst}^{\mathrm{A} * \mathrm{D}}$, om. $\mathrm{PEL}^{\text {var }}$; fratres $64,+$ uobis PEL ${ }^{B}$

libentissime inpendam $\left.5158767778 A M s t^{e d}\right]$ + et inpendar $A M s t^{R}$ et superinpendar $515878 A M s t^{A R}$ ] et ipse super inpendar 61 , et [in]pendar 64, et superinpendam 7576 AMsted, om. 7789 ipse ] om. 616478 , et ipse intendar 7576 , et ipse inpendar $7789 A M s t^{e d}$, ego ipse $\mathrm{AMst}^{\mathrm{R}}$

pro animabus uestris AMst $\left.{ }^{\mathrm{var}}\right]$ animabus uestris 61, pro animis uestris $757^{6}$ AMsted

licet plus uos ] licet uos plus 51, abundantius uos $6177 \mathrm{PEL}^{\mathrm{B}}$, plus uos $\langle 64\rangle$ 7576 , aut plus uos 89 , plurimum uos AMst, plurimum licet ipsis plus uos PEL $^{\text {var }}$

diligens 6r ] diligo AMstrar minus ] licet minus $\mathrm{AMst}^{\mathrm{R}}$

diligar $\mathrm{AMst}^{\mathrm{R}}$ ] diligar a nobis 58 , diligor $6164757677 \mathrm{AMst}^{\mathrm{ed}} \mathrm{PEL}^{\mathrm{B}}$, a uobis diligor 89

12:16 Present: 51545861647576777888 89 AMst PEL

sed esto $\mathrm{AMst}^{\mathrm{R}}$ ] sed ecce 58 , sed 6189 , sit hoc 64 , autem 77 , sed estote ut $88^{\mathrm{C}}$, esto autem $\mathrm{AMst}^{\mathrm{ed}}$, sed ergo esto $\mathrm{PEL}^{\mathrm{B}}$, sed ergo ecce PEL ${ }^{\text {var }}$ ego ] ego autem $77 \mathrm{PEL}^{\mathrm{B}}$, ego sed ego $88^{\mathrm{C}}$

uos non grauaui $\langle 64\rangle \mathrm{AMst}^{\mathrm{R}}$ ] non grauaui uos $61757677 \mathrm{AMst}^{\mathrm{ed}} \mathrm{PEL}^{\mathrm{B}}$ sed cum essem 54 ] sed cum sim 616489 , sed cum sit 75 76, ut AMst astutus dolo $\langle 64\rangle$ uos cepi 788889 ] cepi uos 61, uos genui 64 , uos accepi 757677

12:17 Present: 51545861647576777888 89 AMst PEL numquid 547778 ] an numquid 64, num $\mathrm{AMst}^{\mathrm{R}}$ per aliquem $\langle 64\rangle$ per quem $P E L^{\text {var }}$ eorum ] illorum 5861647789 PEL $^{\mathrm{B}}$, ex his $7576 \mathrm{PEL}^{\mathrm{var}}$ quos misi ad uos 61 ] quod misi ad uos $54^{*}$; + per eum 7576 , + auaritia 77 circumueni uos $\langle 64\rangle$ ] circumuenit uos $89 ;+$ auaritia $78^{g l}$

12:18 Present: 5154586164677576777888 89 AMst PEL rogaui titum 5864 ] rogaui uel adhortatus sum titum 77 et misi cum illo 61 ] et misi cum eo $51545^{8} 64$, et simul misi 77 
fratrem $\langle 64\rangle$ ] fratre $\mathrm{PEL}^{\mathrm{var}} ;+$ nostrum lucam $5^{8}$

numquid $54\langle 67\rangle 77>8$

\pm auaritia 61 , auaritiam 77 , auarus AMst

titus uos circumuenit $\left\langle 88^{*}\right\rangle$ ] titus circumuenit uos $5888^{\mathrm{C}}$, circumuenit uos titus $61\langle 64\rangle 757^{6} 7789$, in uos fuit titus AMst ${ }^{\text {ed }}$, in uobis fuit titus AMst ${ }^{\text {var }}$, uos titus circumuenit PEL $^{\mathrm{B}}$

nonne AMst ${ }^{\text {var }}$ ] non 64678889 AMsted $^{\text {ed }} \mathrm{PL}^{\mathrm{B}}$

eodem spiritu (88) ] eorum spiritu 58 , eo[...] 67

ambulauimus $\langle 64\rangle]$ ambulamus $5^{8} 61 \mathrm{AMst}^{\mathrm{AD}} \mathrm{PEL}^{\mathrm{var}}$, ambulamus uel ambulauimus $\left(77^{*}\right)^{\mathrm{C}}$, ambulemus PEL ${ }^{\text {var }}$; quoniam si uenero iterum non parcam $77^{\dagger}$

nonne $\mathrm{AMst}^{\mathrm{R}}$ ] non 646775767788 89 $\mathrm{AMst}^{\mathrm{ed}}$, non in $\mathrm{PEL}^{\mathrm{B}}$

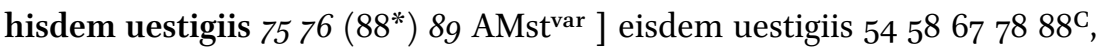
iisdem uestigiis $77 \mathrm{AMst}^{\mathrm{ed}}$

12:19 Present: 5154586164677576777888 89 AMst PEL

olim (61) 88 alt $\mathrm{PEL}^{\mathrm{var}}$ ] iterum $7788 \mathrm{AMst}^{\mathrm{var}} \mathrm{PEL}^{\mathrm{B}}$, om. PEL $^{\mathrm{A}}$

putatis quod $\langle 64\rangle]$ putatis 58 , speratis quoniam 7576

excusemus nos 61 ] excusamus nos 64 PEL var, om. 75 76, nos excusemus 77

apud uos $515^{8}$ ] uobis 647576 ; + disputamus 7576

coram deo $\langle 64\rangle]+$ agimus AMst ${ }^{\text {var }}$

in christo $\mathrm{AMst}^{\mathrm{R}}$ ] om. $757689 \mathrm{AMst}^{\mathrm{ed}}$

loquimur omnia autem ] omnia AMstvar

carissimi $\left.{ }_{51}\langle 67\rangle\right]$ dil $[e c]$ tissimi 64

propter ] pro 64757689 AMst, lac. 67 ; + uel per 77

uestram ] lac. 67, uestra 757689 AMst; + uel uestra 77

aedificationem 51545878 ] aedification [...] 64, aedificatione $757677 \mathrm{AMst}{ }^{\mathrm{ed}}$, exhortatione $\mathrm{AMst}^{\mathrm{var}}$; + et salutem 89

12:20 Present: 5154586164677576777888 89 AMst PEL SPE

timeo enim ] timeo autem $58 \mathrm{PEL}^{\mathrm{B}}$, timeo 61

ne forte cum uenero ] ne cum uenero 7576 AMst; + uel ueniens 77

non quales uolo inueniam uos $\langle 64\rangle]$ non qualis uolo inueniam uos $\mathrm{AMst}^{\mathrm{A}}$ SPEvar

et ego inueniar $\langle 64\rangle]$ ut ego inueniar $54,[\ldots]$ inueniar 67

a uobis AMst ${ }^{\text {var }}$ ] uobis $7576 \mathrm{AMst}^{\mathrm{ed}}$, in uobis $89 \mathrm{PEL}^{\mathrm{var}}$

qualem non ] qualem me non 61, non qualem AMstrar

uultis ne forte ] + sint inter uos $61 \mathrm{AMst}^{\mathrm{PEL}}{ }^{\mathrm{B}}$

$\dagger \quad 2$ Cor. 12:18. The Greek text underneath this addition in VL 77 has been deleted. 
contentiones 5488$]+$ aut $\mathrm{AMst}^{\mathrm{var}}$

aemulationes animositates $51545861\langle 64\rangle 7888$ ] aemulationes PEL ${ }^{\text {var }}$

dissensiones $586177^{*} 88$ ] prouocationes 89 , om. PEL var

detractiones susurrationes $54\langle 64\rangle]$ detractiones 51, susurrationes PEL ${ }^{\text {var }}$

inflationes $54\langle 64\rangle]$ om. $517778 \mathrm{PEL}^{\mathrm{B}}$, tumores 61 AMst, adinflationes PELvar

seditiones ] [...]nes 67, dissensiones 757689

sint inter uos PEL var ] om. 616475767789 AMst PEL $^{\mathrm{B}}$

12:21 Present: 5154586164677576777888 89 AMst PEL SPE

ne ] non 51 , nec PEL ${ }^{\text {** }}$

iterum cum uenero $\langle 64\rangle$ ] iterum ueniente me (77); + ad uos 7576

humiliet me $(64)(78)$

deus ] om. 61; + meus 757677

apud uos $5158\langle 67\rangle]$ ad uos 64, om. 7576

et lugeam ] ut lugeam $5^{1} \mathrm{SPE}^{\mathrm{var}}$, lac. 67 , elugeam $\mathrm{PEL}^{\mathrm{B}}$

multos ex his $58\langle 64\rangle$ ] lac. 67 , multos ex iis $\mathrm{AMst}^{\mathrm{R}}$

qui ante peccauerunt $\langle 67\rangle]$ + peccantium uel peccantibus 77

et non egerunt $\langle 64\rangle]$ lac. 67 , et non 77

paenitentiam $51545^{8} 61\langle 67\rangle 7^{6} 7888$ ] paenitentium uel paenitentiam 77 ; + egerunt uel egentibus 77

super ] om. 757689 , in $77 ;+$ quae gesserunt $\mathrm{PEL}^{\mathrm{B}}$

inmunditia $\left.517888 \mathrm{AMst}^{\mathrm{var}} \mathrm{PEL}^{\mathrm{var}} \mathrm{SPE}^{\mathrm{var}}\right] \mathrm{inm}[. .$.$] 64, lac. 67, om. 7576 \mathrm{PEL}^{\mathrm{B}}$, inmunditiam $89 \mathrm{AMst}^{\text {ed }} \mathrm{PEL}^{\mathrm{A}} \mathrm{SPE}^{\mathrm{ed}}$; + quam gesserunt PEL ${ }^{\text {var }}$

et ] lac. 64, in 7576, om. $\mathrm{PEL}^{\mathrm{B}}$

fornicatione et inpudicitia $\left.515458\langle 67\rangle 7778 \mathrm{AMst}^{\mathrm{var}} \mathrm{PEL}^{\mathrm{var}}\right]$ lac. 64, inmunditiam fornicatione et inpudicitia 7576 , luxuriam et fornicationem 89 , fornicationem et inpudicitiam $\mathrm{AMst}^{\mathrm{ed}} \mathrm{PEL}^{\mathrm{A}}$, fornicationem inpudicitiam $P E L^{B}$, inpudicitia et fornicatione $P E L^{v a r}$, inpudicitiam $S P E^{\text {ed }}$, inpudicitia $\mathrm{SPE}^{\mathrm{var}}$; super inmunditiam $P E L^{\text {var }}$

quam gesserunt ] lac. 6467, om. $\mathrm{PEL}^{\mathrm{B}}$; + et cetera $58,+$ fornicationem et inpudicitiam 89

13:1 Present: $5^{1} 545^{8} 61677576777888$ 89 AMst PEL

ecce ] lac. 67, om. $75767778 \mathrm{AMst}^{\mathrm{ed}} \mathrm{PEL}^{\mathrm{B}}$, en $\mathrm{AMst}^{\mathrm{R}}$

tertio $5154 \mathrm{AMst}^{\mathrm{var}}$ ] lac. 67, tertium $757689 \mathrm{AMst}^{\mathrm{ed}}$, tertium uel tertio 77

hoc ] hic 58 , in hoc 61

uenio ] ueniam 5489

ad uos AMst ${ }^{\text {var }}$ ] apud uel ad uos 77, apud uos AMst ${ }^{\text {ed }}$ + ut $6177^{\mathrm{C}} \mathrm{PEL}^{\text {var }}$ in ore duorum $\left.54\langle 67\rangle \mathrm{AMst}^{\mathrm{AR}}\right]$ in ore duum $\mathrm{AMst}^{\mathrm{ed}}$ 
uel trium testium $\langle 67\rangle \mathrm{AMst}^{\mathrm{var}}$ ] testium et trium $757^{6} 77$, testium uel trium 89 AMst $^{\text {ed }}$ PEL $^{\mathrm{A}}$

stabit ] stet $61 \mathrm{AMst}^{\mathrm{D}}$, stat $\mathrm{AMst}^{\mathrm{A}}$

omne uerbum $\langle 67\rangle]$ omnem uerbum 757689

13:2 Present: 5154586164677576777888 89 AMst PEL

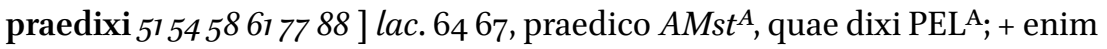
$61 \mathrm{AMst}^{\mathrm{ed}} \mathrm{PEL}^{\mathrm{B}}$

et praedico 515458617788 ] lac. 6467 , et praedixi $A M s t^{A}$ ut praesens $51545867\langle 64\rangle\langle 67\rangle 7788$ ] praesens 757689 , quasi praesens 77 bis ] om. 51 , uobis $5458 \mathrm{PEL}^{\text {var }}$, secundo $616477 \mathrm{PEL}^{\mathrm{B}}$, lac. 67 , secundum 7576

89, secundo aduentu AMsted, secundum aduentum AMst ${ }^{\mathrm{AD}}$, his $\mathrm{PEL}^{\text {var }}$ et nunc absens $\left(64^{*}\right)$ ] lac. 67 , et absens nunc 757677 AMst, nunc et absens

89, et absens $\mathrm{PEL}^{\mathrm{B}}$, et nunc habens PEL ${ }^{\text {var }}$; tertio $89,+$ scribo $\mathrm{AMst}^{\mathrm{R}}$ his $\left.5^{8}\right][\ldots] \mathrm{s} \mathrm{64}$, lac. 67, om. 75 76, iis AMst ${ }^{\mathrm{R}}$ qui ante peccauerunt $][\ldots]$ runt 67 , ante peccauerunt $\mathrm{PEL}^{\mathrm{A} *}$ et ceteris $6 r_{7}$ ] ceteris AMst ${ }^{\mathrm{var}}$ omnibus quoniam si ] omnibus quia si $\langle 64\rangle 757689$ AMst, lac. 67 uenero iterum $\langle 67\rangle]$ iterum uenero $5^{1}$ non parcam $\langle 64\rangle$

13:3 Present: 5154586164677576777888 89 AMst PEL

an ] quia 647576 , quia uel quoniam 77 , quoniam $89 \mathrm{AMst}^{\mathrm{ed}}$, quomodo $\mathrm{AMst}^{\mathrm{R}}$

experimentum $\langle 67\rangle\langle 76\rangle$ ] probationem 64 AMst; + eius PEL $^{\mathrm{B}}$ quaeritis eius $51545^{8}\langle 64\rangle\langle 67\rangle 7_{6} 7888$ ] quaeritis $77 \mathrm{PEL}^{\mathrm{B}}$ qui in me loquitur ] qui me loquitur 54 , loquentis in me 77 christi ] christus 515458647576788889 AMst PEL $^{\mathrm{B}}$, christ[...] 67 qui in ] lac. 67, qui erga $\mathrm{AMst}^{\mathrm{R}}$, quis in $\mathrm{AMst}^{\mathrm{var}}$ uos $\mathrm{AMst}^{\mathrm{R}}$ ] uobis $51546164778889 \mathrm{AMst}^{\mathrm{ed}} \mathrm{PEL}^{\mathrm{B}}$, nobis 58 , lac. 67 non infirmatur $\langle 64\rangle$ ] lac. 67 sed potens est ] sed potens 7576 ; + uel potentur 77 in uobis ] lac. 67

13:4 Present: 5154586164677576777888 89 AMst PEL nam et si $\langle 64\rangle$ ] nam et 7576 , et enim 77 , nam si $P_{E L}{ }^{B}$ crucifixus est ex $\langle 67\rangle$

infirmitate $(64)\langle 67\rangle]$ infirmitate nostra $61 \mathrm{AMst}^{\mathrm{P} E L^{\mathrm{B}}}$, nostra infirmitate PEL $^{\text {var; }}$ + crucifixus est PEL ${ }^{\text {var }}$ sed uiuit ] sed uiuet $64\langle 67\rangle$, uiuit tamen $\mathrm{AMst}^{\mathrm{R}}$ 
ex uirtute dei ] lac. 67

nam et $\langle 67\rangle$ ] et enim $6477 \mathrm{AMst}^{\mathrm{PEL}}{ }^{\mathrm{B}}$, om. 89

nos $\langle 64\rangle]$ si nos 51 61, om. 89, nos si PEL var

infirmi sumus ] infirmamur $6477 \mathrm{AMst}^{\mathrm{ed}}$, inf[...] 67, om. 89, infirmamus AMst ${ }^{\text {var }}$

in illo ] cum illo 6477 78, lac. 67, om. 89

sed uiuemus AMst ${ }^{\text {var }}$ ] sed uiuimus $5^{1} 545^{8} 64 \mathrm{AMst}^{\text {ed }} \mathrm{PEL}$, sed u[...] 67, om. 89

cum $\langle 64\rangle$ ] lac. 67 , in $7576 \mathrm{PEL}^{\mathrm{B}}$, in uel cum 77, om. 89

eo AMst $^{\mathrm{R}}$ ] ipso 6475767778 AMst $^{\text {ed }} \mathrm{PEL}^{\mathrm{B}}$, lac. 67, om. 89, illo AMst ${ }^{\mathrm{var}} \mathrm{PEL}^{\mathrm{A}}$ ex uirtute dei $\langle 67\rangle]$ om. 77 89, in uirtute dei PELvar

in ] om. 64757677 , lac. 67, erga AMst

uobis ] ụobis 51, om. 647576 77, lac. 67, uobismet ipsis 89, uos AMst PEL var

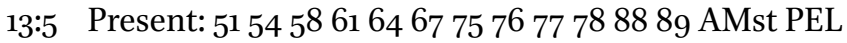

uosmet ipsos temptate $(78)]$ temptate uos $[m]$ et ipsos 64 , lac. 67 , in uobis ipsis temptate 7576 , in uosmet ipsos temptate 77 , temptate 89 , uos ipsos temptate $\mathrm{AMst}^{\mathrm{R}}$

si estis in fide $\langle 67\rangle$

ipsi uos probate $\left(61^{*}\right)\langle 64\rangle$ ] lac. 67 , ipsi uobis probate PELvar

an non $\mathrm{AMst}^{\mathrm{R}}$ ] aut non $6175767789 \mathrm{AMst}^{\mathrm{ed}} \mathrm{PEL}^{\mathrm{B}}$, lac. 67, an 78

cognoscitis ] cognoscẹtis $54^{*}$, agnoscitis 64 , [...] citis 67 , cognoscetis 77

uos ipsos ] uos 6475767789 AMst PEL $^{\mathrm{B}}$, lac. 67, uosmet ipsos PEL var

quia ] quoniam $6177 \mathrm{PEL}^{\mathrm{B}}$, lac. 67 , quod AMst

christus iesus $\langle 64\rangle$ ] iesus christus 617576 , lac. 67

in uobis est nisi ] in nobis est nisi 64 , lac. 67

forte $\left(\mathrm{AMst}^{\mathrm{AD}}\right)$ ] si 757689 , quid 77 , om. AMst $^{\text {ed }}$, si forte PEL var

reprobi $\langle 64\rangle\langle 67\rangle 76]+$ uel inprobi 77

estis $\langle 64\rangle$ ] lac. 67 , essetis PEL var

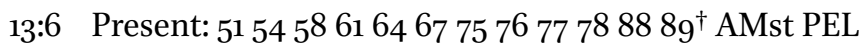

spero autem ] lac. 67 , spero 78 ; + uel enim 77

quod ] quia 64, lac. 67, om. AMst

cognoscetis $\langle 64\rangle\langle 67\rangle$ ] cognoscitis $5^{1} 54^{*} 5^{8} 757^{6} 88^{*} 89$, cognituros uos

AMst

quia ] quoniam 64

$\dagger 2$ Cor. 13:6. This verse is duplicated in VL 89; the text is identical apart from a repetition of estis from the end of the previous verse. 
nos non $\left.\langle 67\rangle \mathrm{AMst}^{\mathrm{AD}}\right]$ non $\mathrm{AMst}^{\mathrm{ed}}$

sumus reprobi $\langle 64\rangle$ ] lac. 67

13:7 Present: $5^{1} 545^{8} 6164677576777888$ 89 AMst PEL CAr SPE

oramus autem ] oro autem 6189 AMst PEL var CAr, [...] autem 67

deum ] ad deum 6477 ,om. $88^{*}$, dominum 89 AMst $^{\text {var }}$ CAr

ut nihil $5^{1} 545^{8}$ ] ne quid 64, ne 75767789 , ne quidquam AMst ${ }^{\text {ed }}$, ut ne quidquam AMst var

mali faciatis $\langle 67\rangle]$ fa[cia]tis mali 64 , faciatis uos quicquam mali 7576 , faciatis uos malum nullum 77 , faciatis quicquam mali 89 , male faciatis CAr, mali faciamus $S \mathrm{PE}^{\mathrm{var}}$

non ut nos ] lac. 67, ut non nos 7576 , abs. CAr

probati $\langle 67\rangle$ ] parati 61, abs. CAr

pareamus ] pereamus $5^{1}$ 89, appareamus $5^{8} 61\langle 64\rangle 77 \mathrm{AMst} \mathrm{PEL}^{\mathrm{A}}$, abs. CAr sed ut uos ] lac. CAr; + faciatis 61

quod bonum est $\mathrm{SPE}^{\mathrm{var}}$ ] lac. 67, bonum 77 , quod iustum est $\mathrm{SPE} \mathrm{ed}^{\mathrm{ed}}$, abs. CAr faciatis $\langle 64\rangle\langle 67\rangle]$ om. 61, facite $\mathrm{SPE}^{\mathrm{var}}$, abs. CAr

nos autem ut ] nos autem 64, nos autem [1-2] ut 89*, nos autem quasi AMst, abs. CAr SPE

reprobi simus $A M s t^{\text {var }}$ ] reprobi sumus 6475767789 * AMst $^{\text {ed }}$ PEL $^{\text {var }}$, reprobi $[\ldots] 67$, abs. CAr SPE

13:8 Present: 5154586164677576777888 89 AMst PEL

non enim $\langle 64\rangle]$ lac. 67

possumus $\langle 67\rangle$ ] possimus 61 , possemus 89 *

aliquid 54 ] quicquid 61, lac. 67 , quid 75767789 , om. $\mathrm{PEL}^{\mathrm{B}}$

aduersus ueritatem ] contra [ue]ritatem 64, lac. 67, aduersum ueritatem 88;

+ aliquid $\mathrm{PEL}^{\mathrm{B}}$

sed pro ueritate (64) (89) ] [...] tate 67

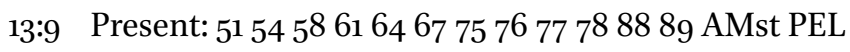

gaudemus $\langle 64\rangle]$ gaudeamus $78 \mathrm{AMst}^{\text {var }}$

enim ] autem 61 $\mathrm{AMst}^{\mathrm{var}} \mathrm{PEL}^{\mathrm{B}}$, lac. 67, etenim AMst ${ }^{\mathrm{var}}$

quando ] quod 61 PEL ${ }^{\text {var }}$, lac. 67, cum $77 \mathrm{AMst} \mathrm{PEL}^{\mathrm{B}}$, quoniam PEL var

nos infirmi sumus $\langle 64\rangle\langle 67\rangle]$ ] nos infirmamur uel infirmi sumus 77, nos infir-

mamur AMst ${ }^{\text {ed }} \mathrm{PEL}^{\mathrm{B}}$, nos infirmamus AMst ${ }^{\mathrm{var}}$

uos autem potentes $\langle 67\rangle \mathrm{AMst}^{\mathrm{D}}$ ] uos autem fortes $6177 \mathrm{AMst}^{\mathrm{ed}} \mathrm{PEL}^{\mathrm{B}}$

estis hoc $\langle 64\rangle$ ] lac. 67 , estis in hoc $\mathrm{AMst}^{\mathrm{R}}$

et ] enim 61, etiam 64, lac. 67, enim et $\mathrm{PEL}^{\mathrm{B}}$

oramus ] precamur $64,[\ldots]$ mus 67 , optamus PEL var; + in AMst ${ }^{\text {ed }}$ 
uestram ] nostram $\mathrm{PEL}^{\mathrm{B}}$

consummationem $\left.545^{8}\langle 67\rangle\right]$ [per]fect [i]onem 64

13:10 Present: $51545^{8} 6164677576777888$ 89 AMst PEL ideo ] lac. 67, propterea 757689 , idcirco AMst; + et 61 haec absens scribo ut $51545861\langle 64\rangle\langle 67\rangle 88$ ] hoc absens scribo ut $\mathrm{PEL}^{\mathrm{B}}$ non praesens $51545861\langle 67\rangle 7788$ ] praesens non 64, praesens PEL var durius ] dure 64, lac. 67 agam ] uta $[r] 64$, lac. 67 , agamus AMst ${ }^{\text {var }}$ secundum potestatem quam $\langle 67\rangle]$ secundum potentiam quam 61 dominus dedit mihi $\left.5^{8}\langle 64\rangle\langle 67\rangle(78) \mathrm{PEL}^{\text {var }}\right]$ dedit dominus nobis PEL ${ }^{\mathrm{A}}$, mihi dedit dominus $\mathrm{PEL}^{\mathrm{B}}$, dedit mihi dominus PEL ${ }^{\mathrm{var}}$ in aedificationem 515458 ] [in] aedification [...] 67, in aedificatione PEL ${ }^{\text {var }}$ et non in ] et non ad 61, lac. 6467 , non in $\mathrm{AMst} \mathrm{AD}^{\mathrm{AD}}$ destructionem $54617888 * 89 *$ ] lac. $6467 ;+$ uestram $6188 *$ PEL $^{\text {var }}$

13:11 Present: 51545861757677788889411 AMst PEL sPE de cetero fratres gaudete 61 ] abs. 411, de cetero fratres $\mathrm{PEL}^{\mathrm{A}}$; + et $\mathrm{PEL}^{\mathrm{B}}$ perfecti estote $\mathrm{SPE}^{\mathrm{var}}$ ] om. $89^{*}$, abs. 411, perficimini $\mathrm{SPE}^{\mathrm{ed}}$; + et $\mathrm{PEL}^{\mathrm{B}}$ exhortamini 54788889 ] consolamini $6177 \mathrm{PEL}^{\mathrm{B}}$, abs. 411, consolationem percipite $\mathrm{AMst}^{\mathrm{ed}}$, consolationem perficite $\mathrm{AMst}^{\text {var }}$; + et consolamini 58 , idem ] id ipsum $617576 \mathrm{PEL}^{\mathrm{B}} \mathrm{SPE}^{\mathrm{var}}$, in id ipsum 89, abs. 411, id est AMst ${ }^{\mathrm{var}}$ SPEvar

sapite pacem habete 54 ] sapite pacifici estote 75767789 , abs. 411, sapite in pace agite AMst

et ] om. PEL var

deus dilectionis et ] deus pacis et 545861757689411 AMst $\mathrm{PEL}^{\mathrm{B}}$, deus 77 pacis ] dilectionis $545^{861411}$ AMst, consolationis $61^{\text {alt }}$, caritatis 7576 , caritas 89 , dilectione $\mathrm{PEL}^{\mathrm{B}}$ erit uobiscum ] erit in uobis $\mathrm{PEL}^{\mathrm{B}}$; + in christo iesu domino nostro 411

13:12 Present: 515458617576777888 89 AMst PEL

salutate inuicem ] salutate uos inuicem 61 AMst, salutate in inuicem 88, om. PEL ${ }^{\mathrm{A}}$

in osculo sancto ] in sancto osculo 7576 , om. PELA salutant uos sancti omnes ] salutant uos omnes sancti $5^{1} 545^{8} 88 \mathrm{AMst}^{\mathrm{A}}$ PELvar

13:13 Present: $5^{1} 545^{8} 61757677788889$ AMst PEL sPM gratia domini nostri AMst ${ }^{\mathrm{var}}$ ] gratia domini $75767789 \mathrm{AMst}^{\mathrm{ed}} \mathrm{SPM}^{\mathrm{var}}$ 


\section{iesu christi et}

caritas dei et $\left(89^{*}\right) \mathrm{SPM}^{\mathrm{var}}$ ] dilectio dei et $\mathrm{AMst} \mathrm{SPM}^{\mathrm{ed}}$, gratia dei et PEL ${ }^{\mathrm{var}}$ communicatio $61 \mathrm{SPM}^{\mathrm{var}}$ ] societas $\mathrm{SPM}^{\mathrm{ed}}$

sancti spiritus ] spiritus sancti AMst ${ }^{\mathrm{var}} \mathrm{PEL}^{\mathrm{var}} \mathrm{SPM}^{\mathrm{var}}$

cum omnibus ] sit cum omnibus $5^{1} 545^{8} 6178 \mathrm{AMst}^{\mathrm{ed}} \mathrm{SPM}^{\mathrm{var}}$

uobis ] nobis $\mathrm{PEL}^{\mathrm{B}}$

amen $\mathrm{AMst}^{\mathrm{R}}$ ] om. $7778 \mathrm{AMst}^{\mathrm{ed}} \mathrm{PEL}^{\mathrm{B}} \mathrm{SPM}$

\section{Explicit}

Present: $5^{1} 545^{86167757677788889}$

explicit epistula ad corinthios secunda $8889^{c}$ ] explicit epistula ad corinthios $5189^{*}$, explicit 54 , om. $5^{861}$, explicit ad corinthios secunda 6778 , ad corinthios secunda explicit 7576 , explicit ad corinthios 77

\pm scripta de macedonia 67 , scribens ut supra sẹ a philippis $75^{c}$ 